\title{
Effective Optical Properties of Ultrathin Gold Coatings Investigated by Tilted Fiber Bragg Grating
}

by

\section{WENJUN ZHOU}

A thesis submitted to the Faculty of Graduate and Postdoctoral Affairs in partial fulfillment of the requirements for the degree of

Doctor of Philosophy

in

Electrical and Computer Engineering

Ottawa-Carleton Institute for Electrical and Computer Engineering

Department of Electronics, Carleton University

Ottawa, Ontario, Canada

January 2015

(C) 2015

Wenjun Zhou 


\section{Abstract}

Tilted fiber Bragg grating (TFBG)-based refractometers are inexpensive, compact, lightweight, and immune to electromagnetic interference and have high chemical resistance. In recent years, many kinds of metal films-coated TFBG refractometers were investigated for enhancing the surrounding refractive index (SRI) measurement sensitivity, resulting from the several orders of magnitude enhancement of the electromagnetic field intensity in the immediate vicinity of various ultrathin metal films or nanostructures. Thus, the measurements of the optical properties of the ultrathin metal coatings (especially gold coatings) on optical fibers are essential for optimizing the TFBG sensing performance.

In this thesis, we firstly measured the properties of the high order cladding modes of standard optical fibers in real-time during the deposition of gold nanoparticle layers by chemical vapour deposition (CVD). Then, the geometry and size-dependent effective medium properties of ultrathin gold CVD films deposited on the bare cladding of single mode optical fibers are characterized by measuring the polarized transmission spectra of TFBGs at near infared wavelengths. This allows the preparation of gold nanoparticle layers that strongly discriminate between radially and azimuthally polarized cladding mode evanescent fields, with important consequences in the plasmonic properties of these layers.

Finally, the optical properties of an ultrathin discontinuous gold evaporation film in different dielectric surroundings are investigated experimentally by measuring the polarizationdependent wavelength shifts and amplitudes of the cladding mode resonances of TFBG. The experimental results are compared to predictions from two widely used effective medium approximations, the generalized Maxwell-Garnett and Bruggeman theories for gold particles in a surrounding matrix.

The results of this thesis show that the TFBG probe can either work as a bulk witness sensor for monitoring the thin film growth on silica substrate or be used as an "ellipsometry" tool for the optical properties measurements of the thin films coated on TFBG surface. 


\section{Acknowledgments}

I would like to thank and acknowledge all the people who I had a chance to work with and made this thesis possible.

My deepest gratitude goes first and foremost to my supervisor Prof. Jacques Albert, who accepted me from China to his group as a $\mathrm{PhD}$ candidate when seldom would have. I have been really fortunate for getting the chance to learn and work with one so kind to everyone but also distinguished on science research. Without his enlightening instruction, impressive patience and consistent encourage, I cannot understand the real research and insist on the high quality of science research. I am grateful to him for indebting me greatly to make the going forward and forward with no fear. I am also grateful to him for walking me through the stages to become a better scientist and a better man.

I would like to especially acknowledge my principal collaborator Mr. David J. Mandia, from Prof. Sean Barry's group in Department of Chemistry, Carleton University, for his contributions to gold depositions, microscopic imaging, and helpful discussions during my $\mathrm{PhD}$ project.

I would like to also acknowledge all colleagues in Prof. Jacques Albert's group, especially for Ms. Albane Laronche, for her instructions and helps in fabricating the TFBGs. Also, I would like to thank Dr. Lingyun Xiong, Dr. Yang Zhang, Dr. Aliaksandr Bialiayeu, Dr. Sandrine Lepinay, and Dr. Hubert Jean-Ruel for their helps in past three years.

Finally, I am sincerely thankful to my family including my parents, grandparents and my girlfriend Yan, who encouraged me throughout the years, and I deeply appreciate their support. 


\section{Contents}

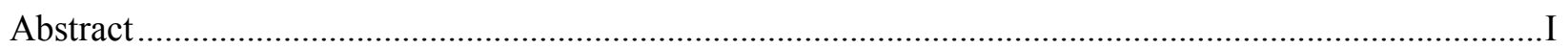

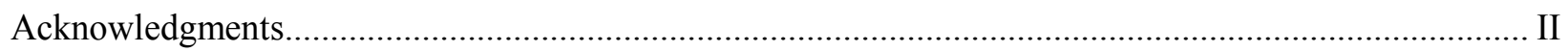

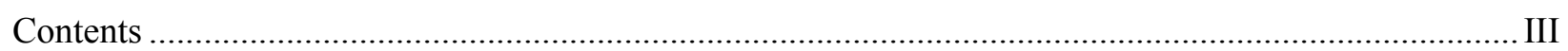

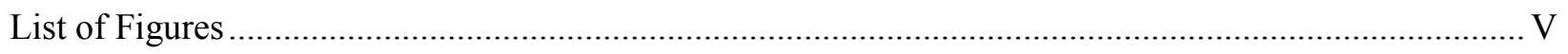

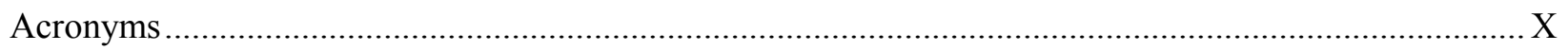

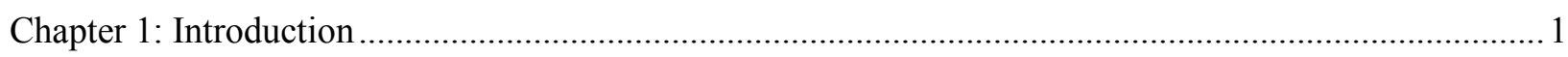

1.1 Measurements of optical properties of ultrathin gold films ....................................................... 1

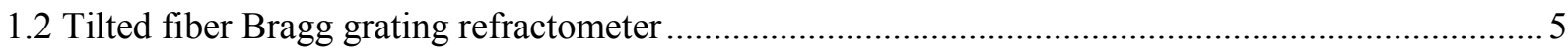

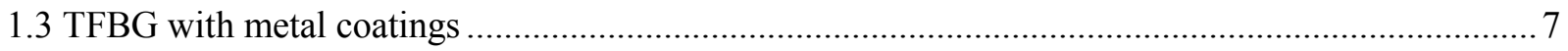

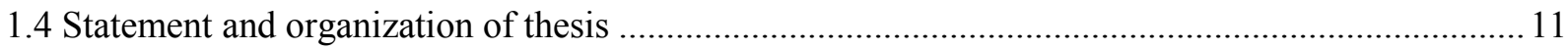

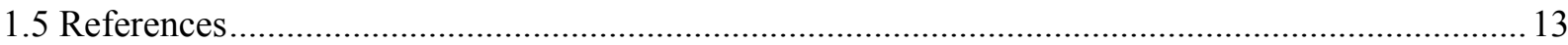

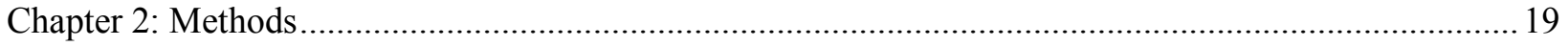

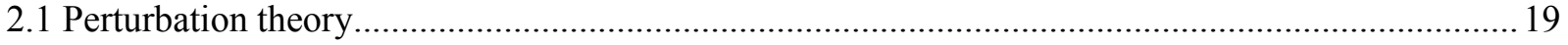

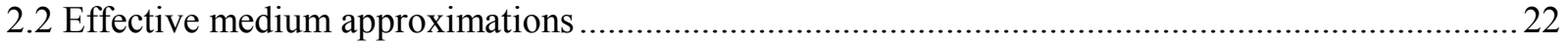

2.3 OptiGrating (by Optiwave) and FIMMWAVE (by Photon Design) .............................................25

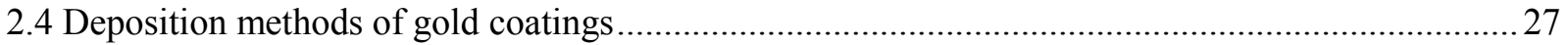

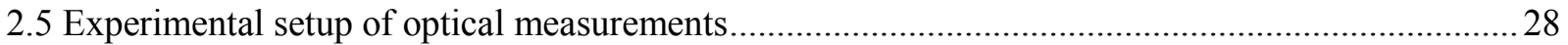

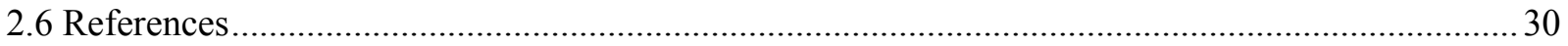

Chapter 3: Polarization-Dependent Properties of the Cladding Modes of a Single Mode Fiber Covered

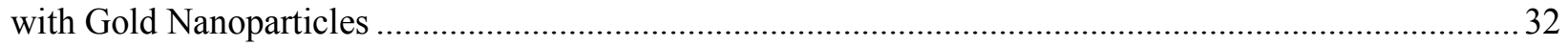

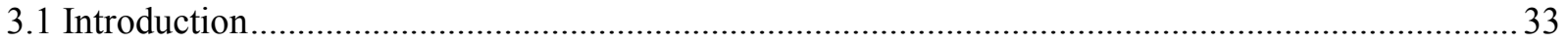

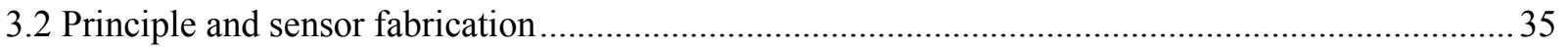

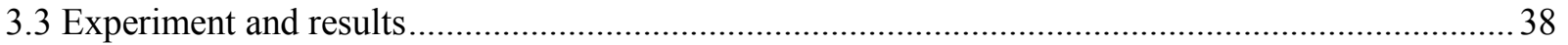

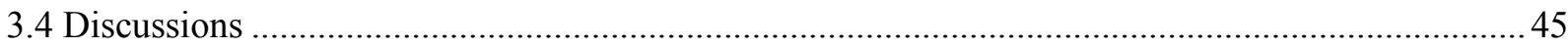

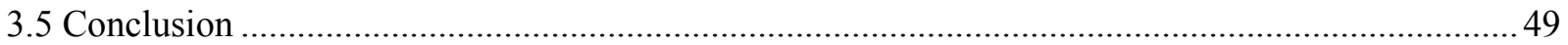

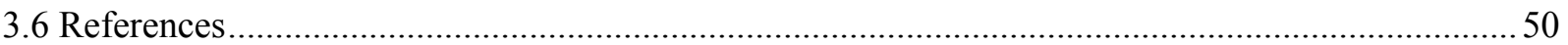

Chapter 4: Effective Permittivity of Ultrathin Chemical Vapor Deposited Gold Films on Optical Fibers at

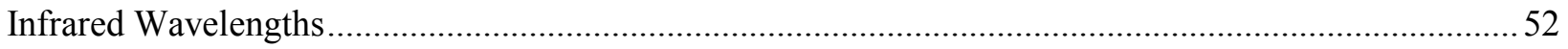

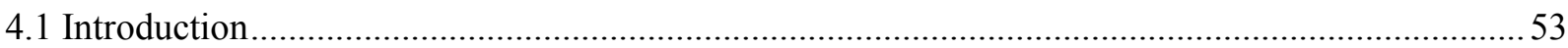

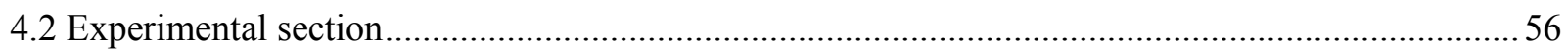

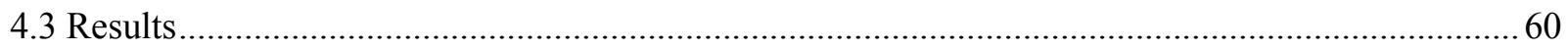

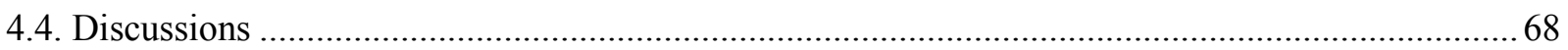




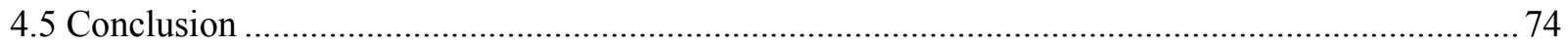

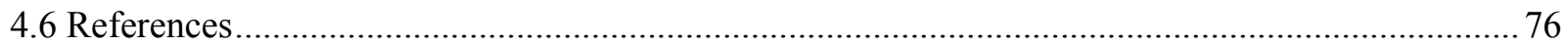

Chapter 5: Anisotropic effective permittivity of an ultrathin gold coating on optical fiber in air, water and

saline solutions

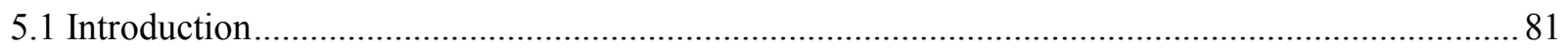

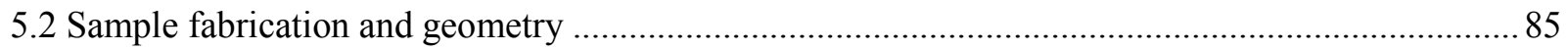

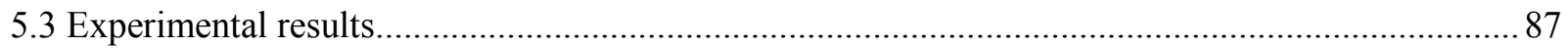

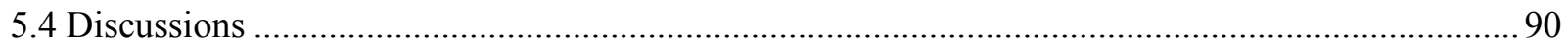

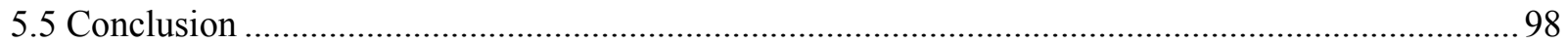

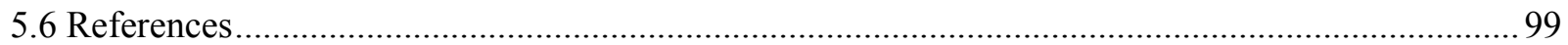

Chapter 6: Optical Anisotropy of Ultrathin Gold Evaporation Coatings on Optical Fibers (Further Work)

103

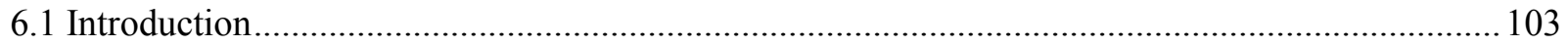

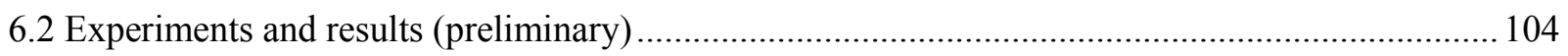

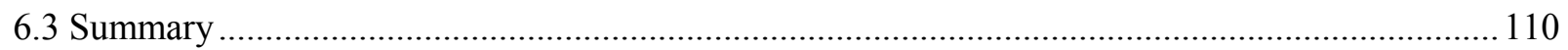

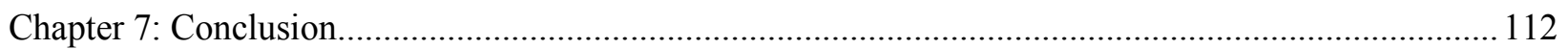




\section{List of Figures}

Fig. 1.1. (a) Schematic diagram of TFBG refractometer immersed in liquid. (b) Schematic diagram of corresponding prism-based bulk refractometry. Arrows A, B, C indicate the leaky mode, cut-off mode, and guided mode, respectively. The SRI is equal to the effective index of the cut-off mode B................. 7 Fig. 1.2. Electric field distributions of four typically vectorial cladding modes ((a) $\mathrm{EH}_{1 \mathrm{~m}}$, (b) $\mathrm{HE}_{1 \mathrm{~m}}$, (c) $\mathrm{TM}_{0 \mathrm{~m}}$, and (d) $\mathrm{TE}_{0 \mathrm{~m}}$ ) coupled from the S- and P-polarized core modes by TFBG, respectively. (Note that the grating planes are parallel to $\mathrm{X}$ axis and tilted away from the $\mathrm{Y}$ axis.) 10

Fig. 2 1. Electric field distributions of TE- and TM-polarized cladding modes with the identical radial orders in a single mode optical fiber.

Fig. 2.2. Schematic drawing of a symmetric three-layer slab waveguide. An additional metal layer results in a permittivity perturbation.

Fig. 2.3. SEM images of $5 \mathrm{~nm}$ thick gold evaporation film (left) and $50 \mathrm{~nm}$ thick gold CVD film (right).

Fig. 2.4. 2D (left) and 3D (right) schematic diagram of spheroidal gold nanoparticles deposited on fiber surface (assuming that the fiber surface of small area $\left(\mu \mathrm{m}^{2}\right)$ is located in the $x y$ plane), interacting with evanescent field of polarized cladding mode. The gold spheroids are oblate so that the major axis $a$ and minor axis (polar axis) $b$ are parallel and perpendicular to the $x y$ plane, respectively.

Fig. 2.5. Spectral evolution (top) of an attenuating cladding mode with increasing imaginary part, comparing with a close cladding mode without attenuation. Fitting curve (bottom) of the cladding mode resonance amplitude versus the corresponding imaginary part. 26

Fig. 2.6. Schematic diagram of experimental setups for TFBG transmission (a) and reflection (b) spectra measurements. The measurement setup is dependent on the style of TFBG probe, which is based on the gold deposition methods. Basically, the reflective TFBG probe with a gold mirror on the end is used in gold CVD; while the normal TFBG sensing head is used in gold evaporation chamber..... .28 Fig. 2.7. Measured $10^{\circ}$ TFBG spectra under TE-polarized, TM-polarized and un-polarized incident lights. The Bragg resonance is around $1610 \mathrm{~nm}$.. 30

Fig. 3.1. Schematic diagram of TFBG coated by gold nanoparticles (the arrows show how the incident core guided light (red) goes through the grating twice, each time coupling light to cladding modes). The light remaining in the core (straight green arrow) returns to the interrogation system.

Fig. 3.2. Simulated electrical fields of a high-order cladding mode excited by P- (left) and S-(right) polarized light. The figures represent a small area of 4 by $4 \mu \mathrm{m}^{2}$ close to cladding boundary (indicated by a horizontal line at $\mathrm{Y}=142.5 \mu \mathrm{m})$. All results were calculated with FIMMWAVE. .36 
Fig. 3.3. Experimental transmission spectra of $10^{\circ} \mathrm{TFBG}$ with S- and P-polarized input light in air, inset: detailed spectra from 1550 to $1556 \mathrm{~nm}$.

Fig. 3.4. Schematic diagram of gold CVD system and spectral monitoring setup. The optical sensing analyzer includes a scanning laser source with a wavelength range from 1520 to $1570 \mathrm{~nm}$ and a synchronized photodetector. .38

Fig. 3.5. TFBG spectral evolutions of during gold nanoparticles deposition under S- (a) and P-polarized (b) light (the color scale represent the amplitude of the resonances). Spectra are acquired every second and temperature-corrected with reference to the Bragg mode shifts. .41

Fig. 3.6. Normalized amplitude (a) and wavelength (b) evolutions versus time of gold deposition under Sand P-polarized light. The vertical dashed lines bound the minimum amplitudes for each polarization, highlighting the simultaneous occurrence of the corresponding wavelength change discontinuities. .42 Fig. 3.7. SEM images of gold nanoparticles deposited on the TFBG surface with different deposition times (with a scale of $500 \mathrm{~nm}$ ). (a) Beginning, (b) middle, and (c) end of TFBG spectral evolution. (d) Sectional image of gold film in a small area with an inset of coated TFBG sectional view...... Fig. 3.8. Reflective transmission spectra and IR-scattering images under S- and P-polarized lights of the coated TFBG with spectral response times of $\sim 20 \mathrm{~s}(\mathrm{a}, \mathrm{b})$ and $40 \mathrm{~s}(\mathrm{c}, \mathrm{d})$.

Fig. 3.9. Simulated intensity distributions of electric fields on single layer gold nanoparticles interacted by $\mathrm{S}$ - (a) and P-polarized (b) light in free space. The incident light propagates along Y-axis in S case, while along $\mathrm{X}$-axis in $\mathrm{P}$ case. .47

Fig. 4.1. Typically reflected transmission spectrum of bare TFBG under TM and TE polarizations.........57 Fig. 4.2. Schematic diagram of experimental process: (a) Gold CVD setup for optical fiber; (b) TFBG spectral measurement system. A source (BBS) followed by a polarization controller generates a broad spectrum of light that is linearly polarized either parallel (TM) or perpendicular (TE) to the tilt plane of the grating. The core-guided light going through the grating is reflected by a gold mirror, goes through the grating a second time and is detected by an optical spectrum analyzer (OSA). .58 Fig. 4.3. (a-d) AFM images of gold CVD films deposited on planar slides with effective thickness of 6, 13, 40, and $65 \mathrm{~nm}$. (e) Two sample height profiles as well as height histograms of the AFM images shown in (a-d). The centers of the effective height distributions are displaced by the average step height of the film for each sample.

Fig. 4.4. (a-f) SEM images of gold CVD films coated on optical fibers at various effective thicknesses (indicated at bottom-left of each image). The scale bar is $500 \mathrm{~nm}$.

Fig. 4.5. Relative wavelength shift (a) and peak-to-peak amplitude attenuation (b) of TE- and TMpolarized cladding mode resonances versus the effective thickness of gold film. The initial peak-to-peak 
amplitudes of the bare gratings are shown as a histogram in Figure 4.5(b). The inset shows the measured spectral changes of the pair of cladding modes used for the $18 \mathrm{~nm}$ thick film.

Fig. 4.6. Cross section of multi-layer model of optical fiber with gold NP film (dash line indicates the average thickness of the gold film) (a) and uniform gold film with equivalent thickness (b).

Fig. 4.7. Real (a) and imaginary (b) parts of the mode effective indices for the TE- and TM-polarized cladding modes measured in Figure 4.5, versus the thickness of the gold film.

Fig. 4.8. Calculated real (a) and imaginary (b) parts of complex refractive indices of gold NP film in TEand TM-polarizations, and corresponding average values versus effective thickness. The dashed line at 25 $\mathrm{nm}$ represents the mean free path of electrons in gold. The horizontal error bar for each thickness is obtained from the standard deviation of the Gaussian fitting curve of the height histograms shown in Table 4.1. 68

Fig. 4.9. Schematic diagram of spheroidal gold nanoparticles deposited on fiber surface (assuming that the fiber surface of small area $\left(\mu \mathrm{m}^{2}\right)$ is located in $x y$ plane), interacting with evanescent field of polarized cladding mode. The gold spheroids are oblate that the major axis $a$ and minor axis (polar axis) $b$ are parallel and perpendicular to $x y$ plane, respectively.

Fig. 4.10. Real (a) and imaginary (b) parts of gold nanoparticle films calculated by the modified ClausiusMossotti (CM) equation versus the measured effective thickness. The inset indicates the metal fraction and aspect ratio used in the CM model. The smaller hollow and solid scattered points (TM/TE-Exp) reproduce (from Figure 4.8) the real and imaginary part of the film index extracted from the measurements.

Fig. 5.1. (a) Schematic diagram of TFBG under X-(S-) and Y-(P-) polarized core modes input. Electric field distributions of four typically vectorial cladding modes ((b) $\mathrm{EH}_{1 \mathrm{~m}}$, (c) $\mathrm{HE}_{1 \mathrm{~m}}$, (d) $\mathrm{TM}_{0 \mathrm{~m}}$, and (e) $\mathrm{TE}_{0 \mathrm{~m}}$ ) coupled from the X-(S-) and Y-(P-) polarized core modes by TFBG, respectively. (Note that the grating planes are parallel to $\mathrm{X}$ axis and tilted away from the $\mathrm{Y}$ axis). The white broken curves indicate the core and cladding edges of optical fiber.

Fig. 5.2. SEM (a) and AFM (b) images of the gold NPs film over an area of $0.5 \times 0.5 \mu \mathrm{m}^{2}$ (with scale bar of $100 \mathrm{~nm}$ ). (c) Histogram of gold NP lateral sizes based on 1172 particles distributed over an area of approximately $1.3 \times 1.0 \mu \mathrm{m}^{2}$. (d) Height histogram of gold NPs film based on $\sim 2.62 \times 10^{5}$ extracted points from $1 \times 1 \mu \mathrm{m}^{2}$ AFM image....... 86

Fig. 5.3. (a) Schematic diagram of experimental setup. Temperature-calibrated spectra of the gold NPscoated (b) and the gold NPs-etched (bare) (c) TFBGs for TE- and TM-polarizations in air, DI water $\left(\mathrm{H}_{2} \mathrm{O}\right)$, and saturated $\mathrm{NaCl}$-water solution $(\mathrm{NaCl})$ surroundings. (The red and blue circles are marked at the positions of the TE- and TM-polarized cladding mode resonances, respectively.) Wavelength separations (d) and peak-to-peak amplitude differences (e) between the pair of polarized cladding modes for the both 
of coated and bare TFBGs under the three SRIs. Note that the signs of the P-P amplitude differences under the cases of bare and coated TFBGs are opposite. 88

Fig. 5.4. 2D schematic diagrams of a mono-layer of oblate gold NPs (a) and effective medium layer that consists of gold and surrounding materials (b) coated on optical fiber surface under evanescent fields of TE- and TM-polarized cladding modes propagating along the $z$-axis. The thickness $t$ of the effective medium layer is equal to the average height $h_{N P}$ of the gold NP film measured from its AFM image. Note that the relative profiles of evanescent field and gold film shown above are not to scale, since the penetration depth of the cladding mode with effective index of $\sim 1.366$ in air is about $130 \mathrm{~nm}$. .90 Fig. 5.5. Real (a) and imaginary (b) parts of the complex effective indices of the TE and TM cladding modes ( $N_{\text {eff }}^{\text {clad }}-i K_{\text {eff }}^{\text {clad }}$ ) under the three SRIs; Real (c) and imaginary (d) parts of complex refractive indices $(n-\mathrm{i} k)$ of the $5.5 \mathrm{~nm}$ thick effective homogeneous gold NPs film for TE and TM modes. The positive and negative errors of the complex refractive indices are evaluated from the thickness uncertainty of $\pm 1 \mathrm{~nm}$ in FIMMWAVE simulations.

Fig. 5.6. Complex refractive indices of the effective medium coating of the three surroundings for (a) outof-plane (TM) and (b) in-plane (TE) directions calculated by M-G and Bruggeman EMAs, compared with the experimentally obtained results and the bulk permittivities of the medium constituents (gold and SRI). The gray and black error bars indicate the deviations of complex refractive indices caused by the minimum aspect ratio $x$ of 4.5/36 and the maximum $x$ of 6.5/20 in EMA models, respectively, based on the average height of $5.5 \mathrm{~nm}( \pm 1 \mathrm{~nm})$ and the average lateral dimensions of $28 \mathrm{~nm}( \pm 8 \mathrm{~nm})$ of the gold NPs. Note that the both of gray and black error bars show in negative direction for the real parts of Bruggeman EMA in Fig. 5.6(a). .96

Fig. 6.1. Diffraction pattern of TFBG induced by red incident light. The white arrows indicate the brightest areas along the diffraction pattern, where the TFBG planes are tilted away from the arrow direction. 105

Fig. 6.2. Polarized spectra of cladding mode resonances in bare and $4 \mathrm{~nm}$ gold-coated $10^{\circ} \mathrm{TFBGs}$ with Bragg wavelength around $1610 \mathrm{~nm}$. The cross sections of two coated optical fibers show the two critical cases of the thickness profiles of the gold coatings relative to the TFBGs' orientations. The pair of arrows in each figure indicates the directions of the gold vapor in the double depositions. 106

Fig. 6.3. SEM images of gold evaporation films deposited on witness wafers (all images were taken under the identical SEM parameters). Each image is marked with the mass-equivalent thickness. The scale bars indicate $100 \mathrm{~nm}$.

Fig. 6.4. Relative wavelength shifts (a) and amplitude attenuations (b) of TE- and TM-polarized cladding mode resonances versus the mass-equivalent thickness of gold film. The initial amplitudes of the pair of cladding modes in bare TFBGs are shown as a histogram in Fig. 6.4(b). 108 
Fig. 6.5. Real (a) and imaginary (b) parts of the mode effective indices for the TE- and TM-polarized cladding modes measured in Fig. 6.4, versus the thickness of the gold film. The simulated complex refractive indices of the two polarized cladding modes for the $8 \mathrm{~nm}$ bulk gold coating are indicated by circles. 


\begin{tabular}{ll} 
Acronyms \\
TFBG & Tilted fiber Bragg grating \\
TE & Transverse electric \\
TM & Transverse magnetic \\
CVD & Chemical vapor deposition \\
PVD & Physical vapor deposition \\
SRI & Surrounding refractive index \\
EMA & Effective medium approximation \\
UV & Ultraviolet \\
NIR & Near infrared radiation \\
SPR & Surface Plasmon resonance \\
SEM & Scanning electron microscopy \\
AFM & Atomic force microscopy \\
BBS & Broadband source \\
OSA & Optical spectrum analyzer \\
PC & Polarization controller \\
\hline
\end{tabular}




\section{Chapter 1: Introduction}

\subsection{Measurements of optical properties of ultrathin gold films}

Gold material with its high metallic conductivity, strong plasmonic activity, and superior chemical stability plays an important role in optoelectronics and nano-optical devices. The electromagnetic field intensity in the immediate vicinity of various thin gold films and nanostructures can be enhanced by several orders of magnitude [1-5]. This phenomenon has led to a number of applications in plasmonic sensing [6,7], surface-enhanced Raman scattering [8], and thermo-plasmonics [9]. Once fabricated, the intensity and wavelength dependence of the plasmonic enhancement are related to the material and geometric parameters of metallic nanostructures: the size [2,3], pattern [4,5], as well as the frequency-dependent permittivities of the gold and surrounding materials $[1,10]$. The linear relative complex permittivity of metal material $\varepsilon_{r}(\omega)=\varepsilon_{1}(\omega)-i \varepsilon_{2}(\omega)$ is a frequency-dependent function, which expresses the fundamental electronic responses to incident light. While size and geometric patterns can be extracted with modern imaging and surface profilometric tools, the complex permittivity is typically obtained from the reflection and transmission measurements of the metallic film with

analysis using Drude model parametrization and Kramers-Krönig relations [11-17]. However, it is now clear that the frequency-dependence of the complex permittivity is inconsistent due to the surface morphology of the thin gold films, even for the bulk films with thickness higher than the mean free path of the conduction electrons $(20-45 \mathrm{~nm})[18,19]$. Based on precise spectroscopic ellipsometry measurements, the frequency-dependent dielectric constant functions of the bulk gold films deposited on different substrates [19] and with different deposition methods [18] can be evaluated. 
In ellipsometry measurements [20], a complex ratio of the Fresnel reflection coefficients is measured from a polarized light with in-plane (s) and out-of-plane (p) components reflected from the sample surface, which can be expressed as,

$$
\rho=r_{p} / r_{s}=\tan (\Psi) e^{i \Delta}
$$

where $\tan (\Psi)$ is the amplitude ratio and $\Delta$ is the phase difference of the reflected p- and spolarized components. For the simplest model of isotropic, homogeneous and semi-infinite thick film, the complex permittivity can be directly calculated from the measured $\Psi$ and $\Delta$,

$$
\varepsilon_{r}(\omega)=\varepsilon_{1}(\omega)-i \varepsilon_{2}(\omega)=\sin ^{2}(\Phi)\left[1+\tan ^{2}(\Phi)\left(\frac{1-\rho}{1+\rho}\right)^{2}\right]
$$

where $\Phi$ is the angle of the incident polarized light. However, for real ellipsometry measurements, a multi-layer model with deduced optical properties and thickness parameters must be established for fitting the experimental ellipsometry data due to the imperfect flatness of the sample surface (typically caused by oxide overlayer and roughness). Furthermore, in order to understand the physical mechanisms behind the complex permittivity and the collective electron response of the gold material, especially for the low-frequency region (including NIR region), the Drude-Sommerfeld free electron model is usually used for explaining the dielectric constant functions of the bulk gold films obtained from the ellipsometry measurements $[18,19]$. In the Drude-Sommerfeld model, gold material is considered as a gas of noninteracting electrons that can have motion driven by an oscillating electric field. The dielectric function can be regarded as the solution of the equation of the motion, which can be written as

$$
\varepsilon_{r}(\omega)=\varepsilon_{\infty}-\frac{\omega_{p}}{\omega^{2}+i \Gamma \omega}
$$


where $\omega_{p}$ is the plasma frequency, $\Gamma$ is the electron relaxation rate, and $\varepsilon_{\infty}$ is the background dielectric function caused by interband transitions. The three effective parameters can be extracted by fitting the calculated dielectric function to the experimental ellipsometry data.

On the other side, these approaches that were developed for bulk (i.e. continuous) metals films are less suitable for ultrathin metal films thinner than the electron mean free path [21] or aggregates of metal nanoparticles (NPs) with individual sizes of the order $50 \mathrm{~nm}$ and less $[22,23]$. In the latter case, it was suggested to modify the collision frequency of free electrons (electron relaxation rate $\Gamma$ ) by an approximate, empirically determined size-dependent parameter in the Drude model [24]. However, it remains difficult to measure the complex permittivity of such nanostructures directly without resorting to various theoretical models for the electromagnetic mixing formulas between the NPs and their surroundings [25]. It is therefore desirable to investigate the permittivity or complex refractive index of ultrathin metal film by several different optical methods. Spectroscopic ellisometry can still obtain the complex permittivity of homogeneous thin films, with decreasing accuracy and resolution as the films get thinner [26,27]. Furthermore, when the films are not isotropic this method becomes significantly less reliable and requires non trivial computations to extract the five parameters of interest (real and imaginary part of the refractive index for TE and TM light, plus the thickness if it is not determined by other means) [28]. Therefore, as the most common tool for investigating the evolution of optical properties of the growing metal films [29-36], especially around the insulator-to-metal transition or percolation threshold (defined as a nominal thickness where the real part of complex permittivity falls from positive to negative during the insulator-to-metal transition) [37,38] (6.5-7 $\mathrm{nm}$ for physical vapor deposition gold films [34]), most researchers have only considered the isotropic situation in the ellipsometry model. Besides, the spectroscopic reflectance or/and 
transmittance technique based on single polarization incidence (TE case) at infrared wavelengths was also reported for investigating the complex permittivity or conductivity of ultrathin gold films around the insulator-to-metal percolation transition (with thickness 1-29 nm), combined with an effective medium approximation (EMA) (mixing formulas) and the Drude model [39-41]. The obtained dielectric functions of the growing thin gold films are only available for the inplane direction due to perpendicular incident light. However, even the EMA models failed in fitting the dielectric functions of the ultrathin gold films around the percolation threshold, measured by the ellipsometry $[34,36]$ and optical reflectance or/and transmittance methods [3941]. It is important to note that a scaling approach was reported for predicting the complex conductivity of the percolating gold films, which shows good agreement with the experimental data over the complete film growth process $[39,40]$.

Moreover, for metallic thin films above the percolation threshold (i.e. for films with a negative real part of the permittivity), the surface plasmon resonance (SPR) technique can also be used to investigate the complex refractive index and thickness of metal films $[42,43]$, but only for TM polarized light and only for a limited range of dielectric refractive indices for the substrate and cover materials of the thin films (for instance, for gold films with thicknesses only from $16.6 \mathrm{~nm}$ to $66.1 \mathrm{~nm}$ [43]). In particular, SPR cannot be excited at an ultrathin metallic filmair interface at infrared wavelengths because the anomalous size-dependent permittivity of the metal becomes positive [44], which occurs below the percolation threshold in gold film growth. In a more recent advance, interferometric picometrology was developed to measure the in-plane (TE) component of the complex refractive index of ultrathin gold films (with thickness $0-10 \mathrm{~nm}$ ) with two individual wavelengths and a linearly polarized light beam at normal incidence [45]. In this new method, the complex index can be calculated from the amplitude change and phase shift 
of the complex reflection coefficient. Therefore, the evolutions of the anisotropic complex permittivities (for both of in-plane and out-of-plane directions) of the ultrathin gold films during the insulator-to-metal transition process have never been reported based on the present measurement techniques.

\subsection{Tilted fiber Bragg grating refractometer}

For decades, optical fibers have been used as sensing elements to measure refractive index of liquids and thin films. In recent years, there has been a growing interest in surrounding refractive index (SRI) sensors, owing to rapid developments in the biological and chemical fields which resulted in great demand for high throughput and accurate acquisition techniques for analyzing biochemical molecules. Optical fiber sensors hold numerous advantages over conventional electrical based sensors. The former are inexpensive, compact, lightweight, and immune to electromagnetic interference and have high chemical resistance. In a conventional optical fiber, light is confined in the fiber core with minimal losses. However, for chemical or SRI sensing using optical fiber, light needs to interact with an analyte in order to obtain its spectral properties. There are two main methods to introduce the SRI-dependence on the properties of the light propagating in the fiber core: 1. Modify the geometrical parameters of the optical fiber waveguide by etching [46], polishing [47], or tapering [48] the cladding layer; 2. Fabricate an infiber core-cladding modal coupler to excite cladding modes that have evanescent fields around the cladding surface [49-51]. While the SRI sensitivity can be achieved with these two methods, the mechanical strength of the optical fiber sensors based on cladding-modified structures and most of the core-cladding modal couplers (such as optical fiber interferometers) are quite low, which limits the stability of the measurement. 
So how to get the core mode light to "see" the SRI changes while keeping the optical fiber durable? Due to the photosensitivity of the germanium-doped silica optical fibers, the refractive index of the fiber core area can be modulated by exposing to UV light. By using the phase-mask method [52], grating planes with specific patterns can be inscribed in the core, which can be divided into two groups of long period gratings and short period gratings. Tilted fiber Bragg grating (TFBG) belongs to the short period grating family, which can couple the forward propagating core mode into backward propagating core mode and cladding modes based on the phase matching condition [53],

$$
\lambda_{c l}=\left(n_{e f f}^{c o}+n_{e f f}^{c l}\right) \Lambda / \cos \theta
$$

where $\lambda_{c l}$ is the wavelength position of the cladding mode resonance, $n_{e f f}^{c o}$ and $n_{e f f}^{c l}$ are the effective indices of the core mode and cladding mode, respectively, $\Lambda$ is the period of the grating planes, and $\theta$ is the tilt angle of the TFBG. Due to the evanescent fields of the cladding modes excited by TFBG, the effective indices of the cladding modes are dependent on the SRI. As shown in Fig. 1.1, the SRI sensing principle of TFBG refractometer can be explained by a simple total internal reflection theory. When the SRI is lower than the effective index of a cladding mode, this cladding mode can be guided in the optical fiber. But when the SRI is increased to the effective index of the cladding mode, the mode becomes cut-off. And if the SRI is larger than the effective index value, the cladding mode becomes lossy and radiates out of the optical fiber waveguide. Because of the large number of cladding modes with different effective indices, the SRI value can be determined by the position of the cut-off mode. The SRI measurement range of the TFBG refractometer lies from 1 to the refractive index of silica of $\sim 1.45$. However, in most publications about TFBG refractometers [54-59], the authors only measured the SRI-induced wavelength shifts or amplitude changes of the guided cladding modes, which aim at determining 
the SRI sensitivity of the modes. It is important to note that these kinds of measurements cannot get the absolute SRI values that also are dependent on the operating wavelength of incident light.

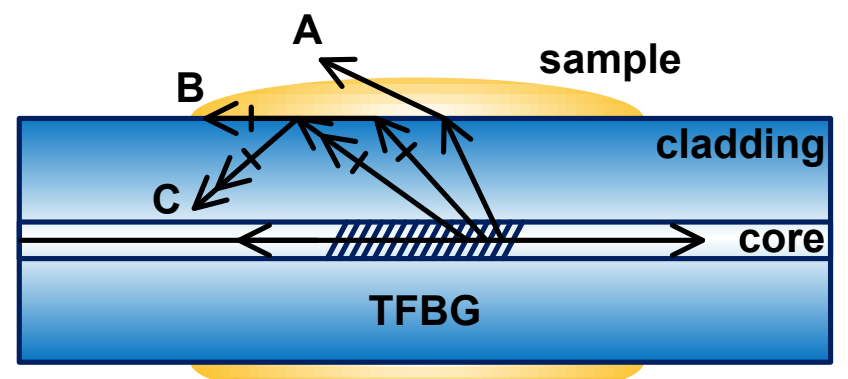

(a)

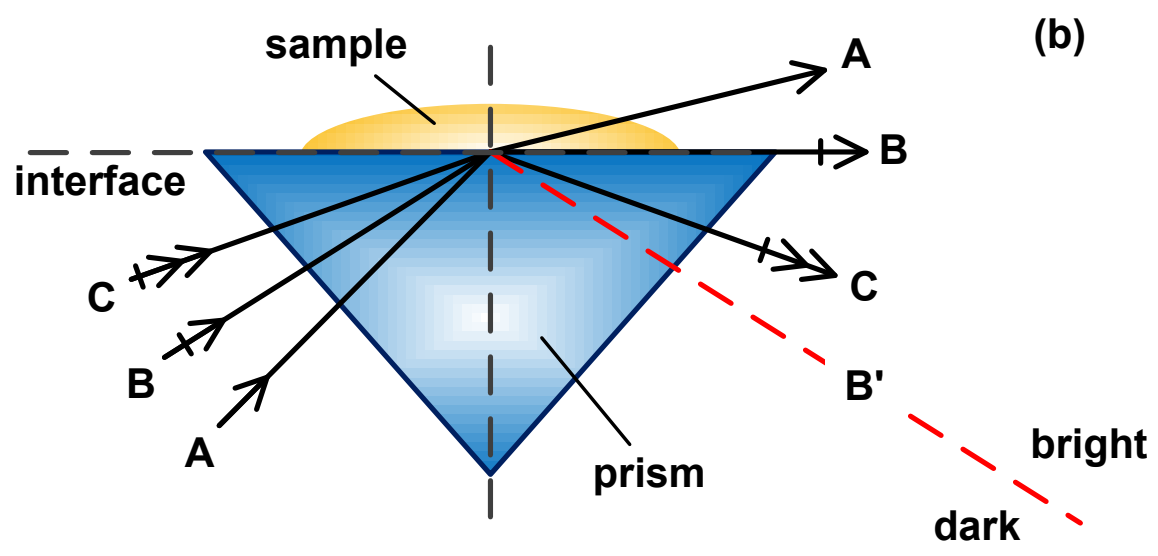

Fig. 1.1. (a) Schematic diagram of a TFBG refractometer immersed in liquid. (b) Schematic diagram of the corresponding prism-based bulk refractometry. Arrows A, B, C indicate the leaky mode, cut-off mode, and guided mode, respectively. The SRI is equal to the effective index of the cut-off mode B.

\subsection{TFBG with metal coatings}

It is expected that depositing metal films on the cladding of an optical fiber will have an impact on the guided cladding modes of such fibers and may lead to new applications in sensing, alloptical switching, and nonlinear optics. TFBGs are ideally suited for such studies because of their capability to excite a number of cladding modes whose properties can be probed very precisely by measuring the wideband spectral response of the grating transmission $[53,60]$. Furthermore, due to the breakup of the circular symmetry caused by the tilted grating planes, the 
polarization state of the incident core mode controls the orientation and polarization of the excited cladding modes at the cladding boundary [53]. This is of utmost importance for metal coatings as the boundary conditions for metal-dielectric interfaces depend very strongly on the polarization state of the light. For example, cladding modes with radial polarization $(\mathrm{P})$ at the cladding boundary can be excited selectively and be used to couple light into surface plasmon waves on metal-coated fibers. A gold-coated TFBG refractometer based on surface plasmon resonance (SPR) was demonstrated using this technique [61], and shown to yield a great enhancement in the minimum surrounding refractive index (SRI) detection level [62], compared to non-TFBG fiber SPR devices $[63,64]$. And it is worthwhile to note that SPR-based optical fiber sensors are mainly used at visible and short infrared wavelengths (less than $800 \mathrm{~nm}$ ), mostly for historical and technological reasons that no longer apply. But high quality commercial optical fibers are now available at very low cost for optical communications with near infared (NIR) wavelengths. Using such fibers in the NIR, TFBG-based SPR sensors can be used for remote sensing applications, in addition to having the advantage of larger penetration of the SPP wave into the surrounding medium and hence potential higher sensitivity [53]. Finally, it was recently demonstrated that by depositing oriented silver nanowires on optical fibers, a localized SPR at communication wavelengths can be excited by cladding modes with azimuthally polarized (S) evanescent electric fields that are parallel to the nanowires [65].

In order to optimize the performance of these TFBG-SPR sensors, the optical properties (complex permittivity) of the thin metal coatings on optical fibers must be measured precisely. However, a common issue with common thin film measurement techniques is that they are not well suited for curved surfaces. For the geometry considered here, a conformal coating on a 125 $\mu \mathrm{m}$ diameter optical fiber, the curvature represents a challenging measurement problem. In 
certain cases, and for very thin films that perturb electromagnetic waves very little, the best way to obtain a high measurement accuracy is to use frequency-domain techniques, such as measuring the frequency shifts of resonator structures that have high quality (Q) factors [66-68]. The curvature of the fiber surface might also be viewed as a problem in the sense that the film could grow differently than on planar substrate, or have different optical properties, thereby negating the possibility of using the TFGB for process monitoring. However, the radius of curvature $(62.5 \mu \mathrm{m})$ and wavelength $(1.5 \mu \mathrm{m})$ are so much larger than the film thicknesses considered that on the scale of the film the fiber surface appears very "flat".

It will be shown in this thesis that very high accuracy measurements of the anisotropic properties of thin metal films can also be obtained with TFBGs, due to the inclination of the grating planes along a specific direction which causes two families of polarized cladding modes with radial or azimuthal electric fields at the fiber cladding boundary to be selectively excited by using linearly polarized input core-guided light $[53,69]$. Fig. 1.2 illustrates the simulated electric fields of four typical polarized cladding modes with the same radial order (number of zeros in field amplitude as a function of radial coordinate) in standard single mode fiber for telecommunications. $\mathrm{TM}_{0 \mathrm{~m}}$ and $\mathrm{EH}_{\mathrm{nm}}$ modes are radially polarized and result from P-polarized core mode input while the $\mathrm{TE}_{0 \mathrm{~m}}$ and $\mathrm{HE}_{\mathrm{nm}}$ modes are azimuthally polarized and result from $\mathrm{S}$ polarized input. P- and S-polarizations refer to the orientation of the input electric field in the plane of incidence and out of the plane of incidence on the tilted grating fringes, respectively. In the following these mode families will be referred to as TM and TE to shorten the text. Due to the orthogonal electric fields at the interface of the fiber cladding with its surroundings, the TMand TE-polarized cladding modes show distinct responses to surrounding refractive index (SRI) changes, but more strongly so when metal coatings or particles are present $[53,70-73]$. 


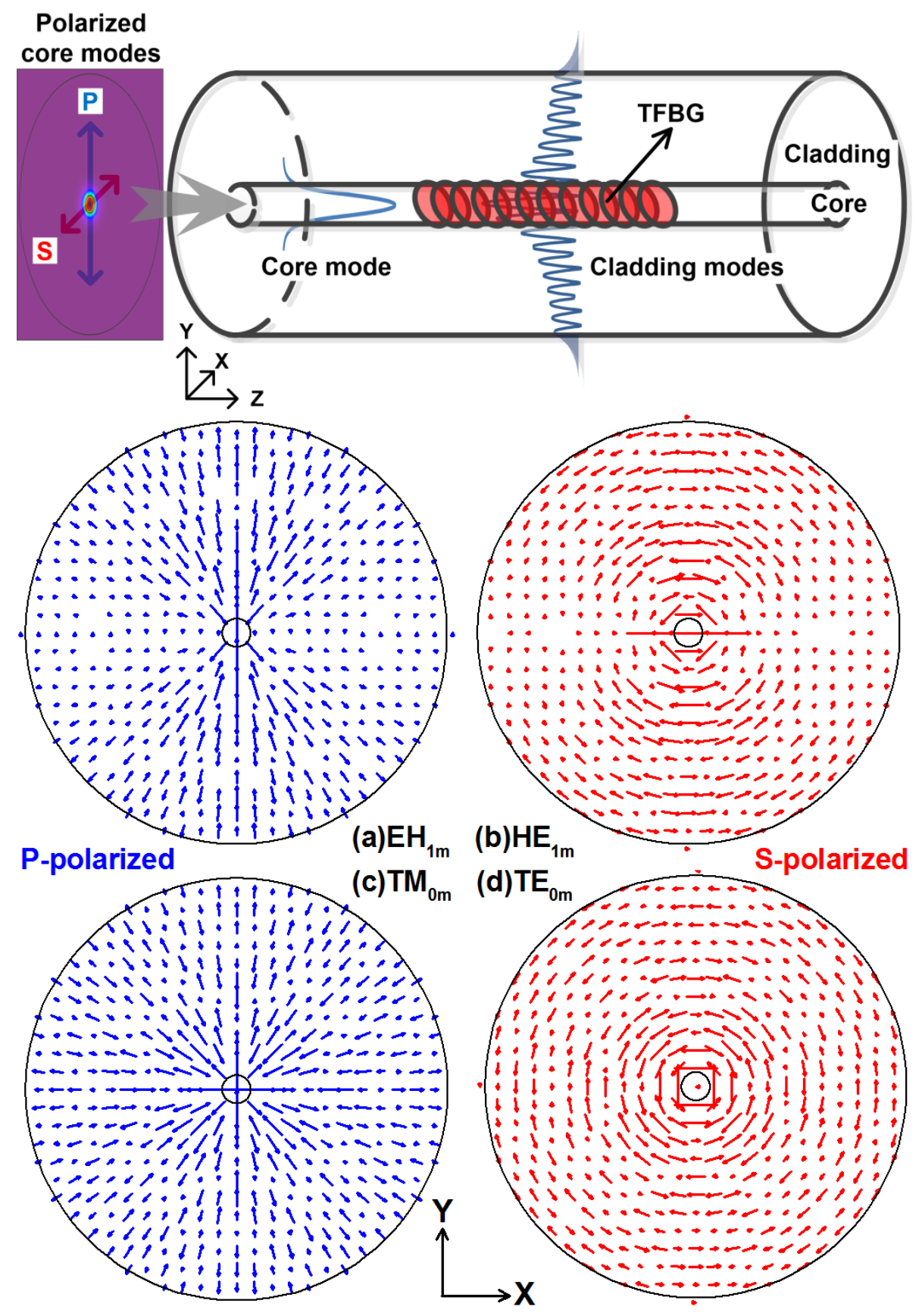

Fig. 1.2. Electric field distributions of four typically vectorial cladding modes ((a) $\mathrm{EH}_{1 \mathrm{~m}}$, (b) $\mathrm{HE}_{1 \mathrm{~m}}$, (c) $\mathrm{TM}_{0 \mathrm{~m}}$, and (d) $\mathrm{TE}_{0 \mathrm{~m}}$ ) coupled from the S- and P-polarized core modes by TFBG, respectively. (Note that the grating planes are parallel to $\mathrm{X}$ axis and tilted away from the $\mathrm{Y}$ axis.) 


\subsection{Statement and organization of thesis}

In this thesis, we firstly investigate the spectral responses of polarized cladding modes, including the wavelength shifts and amplitude changes induced by growing gold films on the optical fiber surface. Then, the polarized evanescent electric field of resonantly excited optical fiber modes are used to probe ultrathin gold films deposited on the surface of the fiber in order to investigate the effective medium properties (refractive index and absorption) of the films at infrared wavelengths. The excitation of the probing optical modes is carried out with a TFBG inscribed in the core of the optical fiber over lengths of several millimeters. The grating couples up to $99 \%$ of the light launched in the core to individual cladding guided modes and thus provides high signal to noise ratio resonances with Q-factors (resonance wavelength/resonance width) of $\sim 10,000$ [53]. Changes in the spectral properties of these cladding mode resonances were used to measure the complex permittivity of the coatings averaged over the full length of the gratings $(5-10 \mathrm{~mm}$ in our experiments). A four-layer cylindrical waveguide model built in FIMMWAVE software (by Photon Design) with vectorial finite differences (FDM)-based complex mode solver was used for this purpose. Compared with the ellipsometry method, the TFBG method further allows the separate measurement of film properties for TE (in-plane electric field) and TM light (out-ofplane) and as a result can detect the presence of birefringence or dichroïsm. These polarizationresolved measurements are obtained by exciting separately cladding-guided modes with azimuthally or radially polarized electric field at the cladding-coating boundary, which correspond respectively to TE and TM light polarizations for the electric field penetrating into the deposited coatings [69].

Chapter 1 presents a short literature review containing the introduction of the measurement methods of the optical properties of the thin metal films, and the background of 
SRI sensing principle and polarization-dependent properties of the TFBG-excited cladding modes.

Chapter 2 mainly introduces the experimental and simulation methods used in the $\mathrm{PhD}$ thesis, including the gold film deposition methods on optical fibers, the simulation models for extracting the effective properties of fiber-optic cladding modes and effective optical properties of the gold coatings, and the effective medium approximation models, such as the MaxwellGarnett mixing formula and the Bruggeman mixing formula.

Chapter 3 reproduces the research article "W. Zhou, et al, Polarization-dependent properties of the cladding modes of a single mode fiber covered with gold nanoparticles, Opt. Express 21(1), 245-255 (2013)." This article shows that the polarized-properties of the high order cladding modes of standard optical fibers can be measured in real-time during the deposition of gold nanoparticle layers by chemical vapour deposition (CVD). These effects are discussed in terms of the evolving metallic boundary conditions perceived by the cladding modes as the nanoparticles grow.

Chapter 4 reproduces the research article "W. Zhou, et al, Effective permittivity of ultrathin chemical vapor deposited gold films on optical fibers at infrared wavelengths, J. Phys. Chem. C 118(1), 670-678 (2014)." It demonstrates that the geometry and size-dependent effective medium properties of ultrathin gold films deposited on the bare cladding of single mode optical fibers by chemical vapour deposition (CVD) can be characterized by measuring the polarized transmission spectra of in-fiber gratings at wavelengths near $1550 \mathrm{~nm}$. And it is found that the CVD gold films are essentially isotropic, apart from a small increasing dichroïsm between the in-plane and out-of-plane component of the imaginary part of the refractive index at thicknesses larger than $25 \mathrm{~nm}$. 
Chapter 5 reproduces the research article "W. Zhou, et al, Anisotropic effective permittivity of an ultrathin gold coating on optical fiber in air, water, and saline solutions, Opt. Express 22(26), 31665-31676 (2014)." In this article, the optical properties of an ultrathin discontinuous gold film (deposited by E-beam evaporation) in different dielectric surroundings are investigated experimentally by measuring the polarization-dependent wavelength shifts and amplitudes of the cladding mode resonances of a TFBG. These results show that the film has stronger dielectric behavior for TM light than for TE, a trend that increases with increasing surrounding index.

Chapter 6 shows some preliminary results about an investigation of the anisotropy of the optical properties the ultrathin gold evaporated films $(3-8 \mathrm{~nm})$ during the insulator-to-metal transition process, based on the polarization-dependent spectral responses of coated TFBGs. It indicates that the optical properties of the gold films in the in-plane direction are metallic, while behaving as an insulator in the out-of-plane direction. Therefore, the percolation threshold of the growing gold evaporated film occurs at a lower thickness value for the in-plane direction than the out-of-plane direction. This investigation will be continued as future work.

Chapter 7 concludes the main results proposed in this thesis, the remaining issues of the TFBG model for calculating the complex permittivity of the metal coatings, and discusses the potential solutions for future work.

\subsection{References}

1. C. Ciracì, R. T. Hill, J. J. Mock, Y. Urzhumov, A. I. Fernández-Domínguez, S. A. Maier, J. B. Pendry, A. Chilkoti, D. R. Smith, "Probing the Ultimate Limits of Plasmonic Enhancement," Science 337, 1072-1074 (2012).

2. T. Chen, M. Pourmand, A. Feizpour, B. Cushman, B. M. Reinhard, "Tailoring Plasmon Coupling in Self-Assembled One-Dimensional Au Nanoparticle Chains through Simultaneous Control of Size and Gap Separation,” J. Phys. Chem. Lett. 4, 2147-2152 (2013). 
3. C. Deeb, X. Zhou, J. Plain, G. P. Wiederrecht, R. Bachelot, M. Russell, P. K. Jain, "Size Dependence of the Plasmonic Near-Field Measured via Single-Nanoparticle Photoimaging,” J. Phys. Chem. C 117, 10669-10676 (2013).

4. T. Jägeler-Hoheisel, J. Cordeiro, O. Lecarme, A. Cuche, C. Girard, E. Dujardin, D. Peyrade, A. Arbouet, "Plasmonic Shaping in Gold Nanoparticle Three-Dimensional Assemblies," J. Phys. Chem. C 117, 23126-23132 (2013).

5. B. Yan, S. V. Boriskina, B. M. Reinhard, “Optimizing Gold Nanoparticle Cluster Configurations (n $\leq$ 7) for Array Applications,” J. Phys. Chem. C 115, 4578-4583 (2011).

6. J. N. Anker, W. P. Hall, O. Lyandres, N. C. Shah, J. Zhao, R. P. Van Duyne, "Biosensing with Plasmonic Nanosensors," Nat. Mater. 7, 442-453 (2008).

7. J. J. Mock, R. T. Hill, Y.-J. Tsai, A. Chilkoti, D. R. Smith, "Probing Dynamically Tunable Localized Surface Plasmon Resonances of Film-Coupled Nanoparticles by Evanescent Wave Excitation," Nano Lett. 12, 1757-1764 (2012).

8. S. Sarkar, M. Pradhan, A. K. Sinha, M. Basu, T. Pal, "Chelate Effect in Surface Enhanced Raman Scattering with Transition Metal Nanoparticles,” J. Phys. Chem. Lett. 1, 439-444 (2010).

9. G. Baffou, R. Quidant, "Thermo-Plasmonics: Using Metallic Nanostructures as Nano-Sources of Heat,” Laser Photon. Rev. 7, 171-187 (2013).

10. G. H. Cross, "Fundamental Limit to the Use of Effective Medium Theories in Optics," Opt. Lett. 38, 3057-3060 (2013).

11. P. B. Johnson, R. W. Christy, “Optical Constants of the Noble Metals," Phys. Rev. B 6, 4370-4379 (1972).

12. M. A. Ordal, L. L. Long, R. J. Bell, S. E. Bell, R. R. Bell, Jr., R. W. Alexander, C. A. Ward, “Optical Properties of the Metals $\mathrm{Al}, \mathrm{Co}, \mathrm{Cu}, \mathrm{Au}, \mathrm{Fe}, \mathrm{Pb}, \mathrm{Ni}, \mathrm{Pd}, \mathrm{Pt}, \mathrm{Ag}, \mathrm{Ti}$, and $\mathrm{W}$ in the Infrared and Far Infrared," Appl. Opt. 22, 1099-1119 (1983).

13. P. Grosse, V. Offermann, “Analysis of Reflectance Data Using the Kramers-Kronig Relations," Appl. Phys. A 52, 138-144 (1991).

14. L. G. Schulz, "The Optical Constants of Silver, Gold, Copper, and Aluminum. I. The Absorption Coefficient $k$,” J. Opt. Soc. Am. 44, 357-362 (1954).

15. L. G. Schulz and F. R. TANGHERLINI, "Optical Constants of Silver, Gold, Copper, and Aluminum. II. The Index of Refraction n,” J. Opt. Soc. Am. 44, 362-367 (1954).

16. J. E. Nestell, R. W. Christy, "Derivation of Optical Constants of Metals from Thin-Film Measurements at Oblique Incidence," Appl. Opt. 11, 643-651 (1972).

17. G. Brandli, A. J. Sievers, "Absolute Measurement of the Far-Infrared Surface Resistance of Pb," Phys. Rev. B 5, 3550 (1972). 
18. R. L. Olmon, B. Slovick, T. W. Johnson, D. Shelton, S.-H. Oh, G. D. Boreman, and M. B. Raschke, “Optical dielectric function of gold," Phys. Rev. B 86, 235147 (2012).

19. J. Trollmann, A. Pucci, "Infrared dielectric function of gold films in relation to their morphology," J. Phys. Chem. C 118, 15011-15018 (2014).

20. H. Fujiwara, Spectroscopic Ellipsometry: Principles and Applications; Wiley: England, 2007.

21. J. J. Tu, C. C. Homes, and M. Strongin, "Optical Properties of Ultrathin Films: Evidence for a Dielectric Anomaly at the Insulator-to-Metal Transition,” Phys. Rev. Lett. 90, 017402 (2003).

22. U. Kreibig, and M. Vollmer, Optical Properties of Metal Clusters; Springer: Berlin, Germany, 1995.

23. A. Manjavacas, and F. J. García de Abajo, “Tunable plasmons in atomically thin gold nanodisks," Nat. Commun. 5, 3548 (2014).

24. U. Kreibig, C. V. Fragstein, "The Limitation of Electron Mean Free Path in Small Silver Particles,” Z. Physik 244, 307-323 (1969).

25. A. Sihvola, Electromagnetic Mixing Formulas and Applications; The Institution of Electrical Engineers: London, U.K., 1999.

26. M. Yamamoto, T. Namioka, "In situ Ellipsometric Study of Optical Properties of Ultrathin Films" Appl. Opt. 31, 1612-1621 (1992).

27. M. Chen, R. G. Horn, "Refractive Index of Sparse Layers of Adsorbed Gold Nanoparticles," J. Colloid Interface Sci. 315, 814-817 (2007).

28. http://www.jawoollam.com/an_anisotropy.html

29. H. V. Nguyen, I. An, R. W. Collins, "Evolution of the optical functions of thin-film aluminum: A real-time spectroscopic ellipsometry study,” Phys. Rev. B 47, 3947-3965 (1993).

30. T. W. H. Oates, D. R. McKenzie, and M. M. M. Bilek, "Percolation threshold in ultrathin titanium films determined by in situ spectroscopic ellipsometry," Phys. Rev. B 70, 195406 (2004).

31. T. W. H. Oates, and A. Mücklich, "Evolution of plasmon resonances during plasma deposition of silver nanoparticles," Nanotechnology 16, 2606-2611 (2005).

32. Anna Jo de Vries, E. Stefan Kooij, H. Wormeester, A. A. Mewe, and B. Poelsema, "Ellipsometric study of percolation in electroless deposited silver films,” J. Appl. Phys. 101, 053703 (2007).

33. T. Brandt, M. Hövel, B. Gompf, and M. Dressel, "Temperature- and frequency-dependent optical properties of ultrathin Au films," Phys. Rev. B 78, 205409 (2008).

34. M. Hovel, B. Gompf, and M. Dressel, "Dielectric properties of ultrathin metal films around the percolation threshold," Phys. Rev. B 81, 035402 (2010).

35. H. T. Beyene, J. W. Weber, M. A. Verheijen, M. C. M. van de Sanden, and M. Creatore, "Real Time In Situ Spectroscopic Ellipsometry of the Growth and Plasmonic Properties of Au Nanoparticles on $\mathrm{SiO}_{2}$," Nano Res. 5, 513-520 (2012). 
36. X. D. Li, T. P. Chen, Y. Liu, and K. C. Leong, "Influence of localized surface plasmon resonance and free electrons on the optical properties of ultrathin Au films: a study of the aggregation effect," Opt. Express 22, 5124-5132 (2014).

37. A. L. Efros, and B. I. Shklovskii, "Critical behaviour of conductivity and dielectric constant near the metal-non-metal transition threshold," Phys. Stat. Sol. (B) 76, 475-485 (1976).

38. X. Yu, P. M. Duxbury, G. Jeffers, and M. A. Dubson, "Coalescence and percolation in thin metal films,” Phys. Rev. B 44(23), 13163-13166 (1991).

39. Y. Yagil, M. Yosefin, D. J. Bergman, G. Deutscher, and P. Gadenne, "Scaling theory for the optical properties of semicontinuous metal films," Phys. Rev. B 43(13), 11342-11352 (1991).

40. Y. Yagil, P. Gadenne, C. Julien, and G. Deutscher, "Optical properties of thin semicontinuous gold films over a wavelength range of 2.5 to $500 \mu \mathrm{m}$," Phys. Rev. B 46(4), 2503-2511 (1992).

41. M. Walther, D. G. Cooke, C. Sherstan, M. Hajar, M. R. Freeman, and F. A. Hegmann, "Terahertz conductivity of thin gold films at the metal-insulator percolation transition," Phys. Rev. B 76, 125408 (2007).

42. T. Inagaki, J. P. Goudonnet, P. Royer, E. T. Arakawa, "Optical Properties of Silver Island Films in the Attenuated-Total-Reflection Geometry,” Appl. Opt. 25, 3635-3639 (1986).

43. W.-J. Lee, J.-E. Kim, H.-Y. Park, S. Park, M.-S. Kim, “Optical Constants of Evaporated Gold Films Measured by Surface Plasmon Resonance at Telecommunication Wavelengths,” J. Appl. Phys. 103, 073713 (2008).

44. J. Homola, Electromagnetic Theory of Surface Plasmons. Springer Ser. Chem. Sens. Biosens. 4, 3-44 (2006).

45. X. Wang, K.-P. Chen, M. Zhao, D. D. Nolte, "Refractive Index and Dielectric Constant Evolution of Ultra-Thin Gold from Clusters to Films,” Opt. Express 18, 24859-24867 (2010).

46. A. Iadicicco, A. Cusano, A. Cutolo, R. Bernini, and M. Giordano, "Thinned fiber Bragg gratings as high sensitivity refractive index sensor," IEEE Photon. Technol. Lett. 16(4), 1149-1151 (2004).

47. W. Dong, and R. Zhao, "Detection of liquid-level variation using a side-polished fiber Bragg grating," Opt. Laser Technol. 42, 214-218 (2010).

48. L.-Y. Shao, A.P. Zhang, W.-S. Liu, H.-F. Fu, and S. He, "Optical refractive-index sensor based on dual fiber-Bragg gratings interposed with a multimode-fiber taper," IEEE Photon. Technol. 19(1), 3032 (2007).

49. Q. Wu, Y. Semenova, P. Wang, and G. Farrell, "High sensitive SMS fiber structure based refractometer - analysis and experiment," Opt. Express 19(9), 7937-7944 (2011).

50. R. Jha, J. Villatoro, G. Badenes, and V. Pruneri, "Refractometry based on a photonic crystal fiber interferometer," Opt. Lett. 34(5), 617-619 (2009). 
51. W.C. Wong, C. C. Chan, Y. F. Zhang, and K. C. Leong, "Miniature single-mode fiber refractive index interferometer sensor based on high order cladding mode and core-offset," IEEE Photon. Technol. Lett. 24(5), 359-361 (2012).

52. K. O. Hill, B. Malo, F. Bilodeau, D. C. Johnson, and J. Albert, "Bragg gratings fabricated in monomode photosensitive optical fiber by UV exposure through a phase mask,” Appl. Phys. Lett. 62 (10), 1035-1037 (1993).

53. J. Albert, L.-Y. Shao and C. Caucheteur, “Tilted fiber Bragg grating sensors,” Laser Photon. Rev. 7 , 83-108 (2013).

54. C.-F. Chan, C. Chen, A. Jafari. A. Laronche, D. J. Thomson and J. Albert, "Optical fiber refractometer using narrowband cladding-mode resonance shifts,” Appl. Opt. 46, 1142-1149 (2007).

55. T. Guo, C. Chen, A. Laronche, and J. Albert, "Power-referenced and temperature-calibrated optical fiber refractometer," IEEE Photon. Technol. Lett. 20(8), 635-637 (2008).

56. T. Guo, H.-Y. Tam, P. A. Krug, and J. Albert, "Reflective tilted fiber Bragg grating refractometer based on strong cladding to core recoupling,” Opt. Express 17(7), 5736-5742 (2009).

57. C. Caucheteur, P. Mégret, and A. Cusano, "Tilted Bragg grating multipoint sensor based on wavelength-gated cladding modes coupling," Appl. Opt. 48(20), 3915-3920 (2009).

58. Y. Jin, X. Dong, H. Gong, and C. Shen, "Refractive-index sensor based on tilted fiber Bragg grating interacting with multimode fiber," Microwave Opt. Technol. Lett. 52(6), 1375-1377 (2010).

59. Y. Miao, B. Liu, S. Tian, and Q. Zhao, "Temperature-insensitive refractive index sensor based on tilted fiber Bragg grating,” Microwave Opt. Technol. Lett. 51(2), 479-483 (2009).

60. Y.-C. Lu, R. Geng, C. Wang, F. Zhang, C. Liu, T. Ning and S. Jian, "Polarization effects in tilted fiber Bragg grating refractometers,” J. Lightwave Technol. 28, 1677-1684 (2010).

61. Y. Shevchenko, C. Chen, M. A. Dakka and J. Albert, "Polarization-selective grating excitation of plasmons in cylindrical optical fiber," Opt. Lett. 35, 637-639 (2010).

62. C. Caucheteur, Y. Shevchenko, L.-Y. Shao, M. Wuilpart and J. Albert, "High resolution interrogation of tilted fiber grating SPR sensors from polarization properties measurement," Opt. Express 19, 16561664 (2011).

63. J. Pollet, F. Delport, K. P. F. Janssen, K. Jans, G. Maes, H. Pfeiffer, M. Wevers and J. Lammertyn, "Fiber optic SPR biosensing of DNA hybridization and DNA-protein interactions," Biosens. Bioelectron. 25, 864-869 (2009).

64. T. Schuster, R. Herschel, N. Neumann and C. G. Schaeffer, "Miniaturized long-period fiber grating assisted surface plasmon resonance sensor,” J. Lightwave Technol. 30, 1003-1008 (2012).

65. J.-M. Renoirt, M. Debliquy, J. Albert, A. Ianoul, and C. Caucheteur, "Surface plasmon resonances in oriented silver nanowire coatings on optical fiber," J. Phys. Chem. C 118, 11035-11042 (2014). 
66. I. M. White, X. Fan, “On the Performance Quantification of Resonant Refractive Index Sensors," Opt. Express, 16, 1020-1028 (2008).

67. H. Zhu, I. M. White, J. D. Suter, M. Zourob, X. Fan, "Integrated Refractive Index Optical Ring Resonator Detector for Capillary Electrophoresis," Anal. Chem. 79, 930-937 (2007).

68. J. Albert, S. Lepinay, C. Caucheteur, M. C. DeRosa, "High Resolution Grating-Assisted Surface Plasmon Resonance Fiber Optic Aptasensor,” Methods 63, 239-254 (2013).

69. M. Z. Alam, and J. Albert, "Selective excitation of radically and azimuthally polarized optical fiber cladding modes," J. Lightwave Technol. 31, 3167-3175 (2013).

70. C. Caucheteur, C. Chen, V. Voisin, P. Berini and J. Albert, "A thin metal sheath lifts the EH to HE degeneracy in the cladding mode refractometric sensitivity of optical fiber sensors," Appl. Phys. Lett. 99, 041118 (2011).

71. W. Zhou, D. J. Mandia, M. B. E. Griffiths, A. Bialiayeu, Y. Zhang, P. G. Gordon, S. T. Barry, and J. Albert, "Polarization-dependent properties of the cladding modes of a single mode fiber covered with gold nanoparticles," Opt. Express 21, 245-255 (2013).

72. W. Zhou, D. J. Mandia, M. B.E. Griffiths, S. T. Barry, and J. Albert, "Effective permittivity of ultrathin chemical vapor deposited gold films on optical fibers at infrared wavelengths," J. Phys. Chem. C 118(1), 670-678 (2014).

73. W. Zhou, D. J. Mandia, S. T. Barry, and J. Albert, "Anisotropic effective permittivity of an ultrathin gold coating on optical fiber in air, water, and saline solutions," Opt. Express 22(26), 31665-31676 (2014). 


\section{Chapter 2: Methods}

In this chapter, we introduce the main methods used in this thesis, including theories, simulation models, and experimental methods.

\subsection{Perturbation theory}

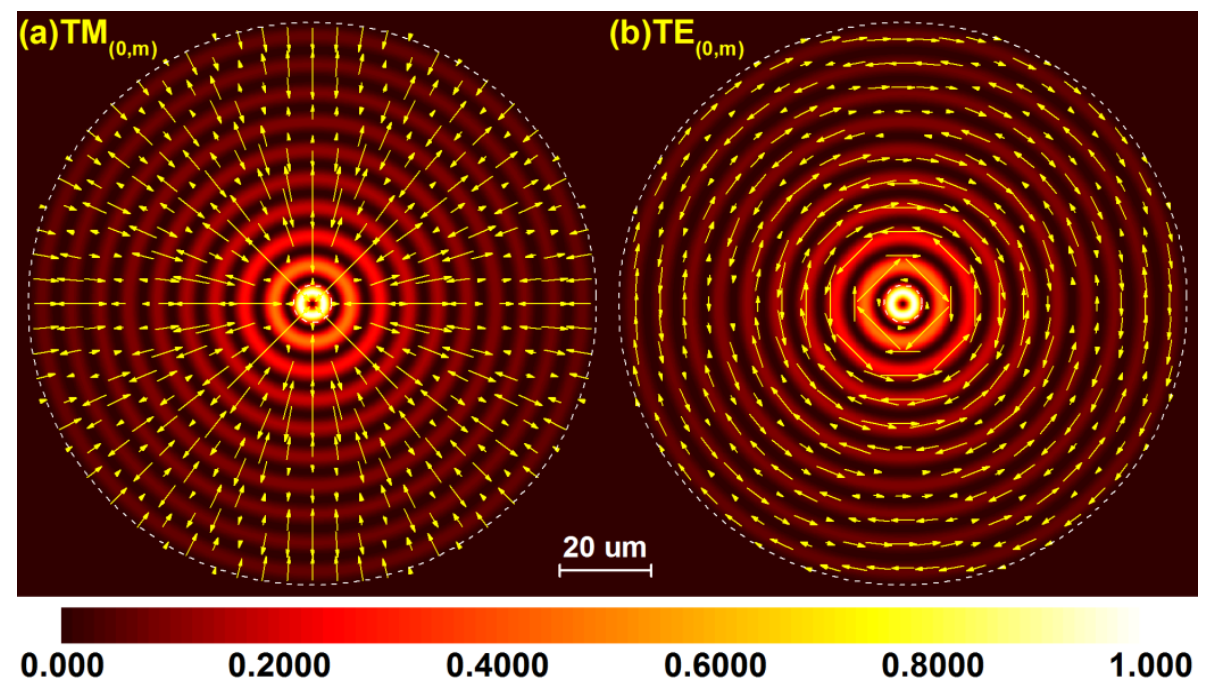

Fig. 2 1. Electric field distributions of TE- and TM-polarized cladding modes with identical radial orders in a single mode optical fiber.

In order to understand the polarization-dependent spectral responses of the TE- and TMpolarized cladding modes caused by ultrathin gold coatings, a simple perturbation theory for simulating the polarized attenuations in a slab optical waveguide induced by a small permittivity change $\Delta \varepsilon$ resulting from the gold coating is applied. Fig. 2.1 shows the $2 \mathrm{D}$ electric field distributions of a pair of TE and TM cladding modes with an identical radial order of 10, where the electrical fields follow the perpendicular and parallel directions with respect to the interface of cladding and surrounding. Based on the centrally symmetrical distributions of the electric fields, we can consider a simplified model of the optical fiber waveguide as a three-layer slab waveguide (as shown in Fig. 2.2) supporting a pair of TE and TM modes, where the $y$-direction 
has no variation. Based on Maxwell's equations and the boundary conditions, the wave equations (scalar eigenvalue equations) of the TE and TM modes can be respectively expressed as,

$$
\begin{gathered}
\frac{\partial^{2}}{\partial x^{2}} E_{y}(x)+\left[k_{0}^{2} \varepsilon(x)-\beta^{2}\right] E_{y}(x)=0 \\
\frac{\partial^{2}}{\partial x^{2}} H_{y}(x)-\frac{1}{\varepsilon(x)} \frac{\partial \varepsilon(x)}{\partial x} \frac{\partial}{\partial x} H_{y}(x)+\left[k_{0}^{2} \varepsilon(x)-\beta^{2}\right] H_{y}(x)=0
\end{gathered}
$$

where $\beta$ is the propagation constant, $k_{0}=\omega / c$ is the wavenumber in vacuum, $\varepsilon(x)$ is the relative permittivity of the waveguide materials, $E_{y}$ and $H_{y}$ are the electric and magnetic components of the TE and TM modes in y direction, respectively. And the $E_{y}$ and $H_{y}$ functions can be expressed similarly as,

$$
\begin{aligned}
& E_{y}(x, z, t)=E_{m}(x) \exp [i(\omega t-\beta z)] \\
& H_{y}(x, z, t)=H_{m}(x) \exp [i(\omega t-\beta z)]
\end{aligned}
$$

where $E_{m}(x)$ and $H_{m}(x)$ are the transverse spatial shapes, or eigenfunctions of the guided modes.

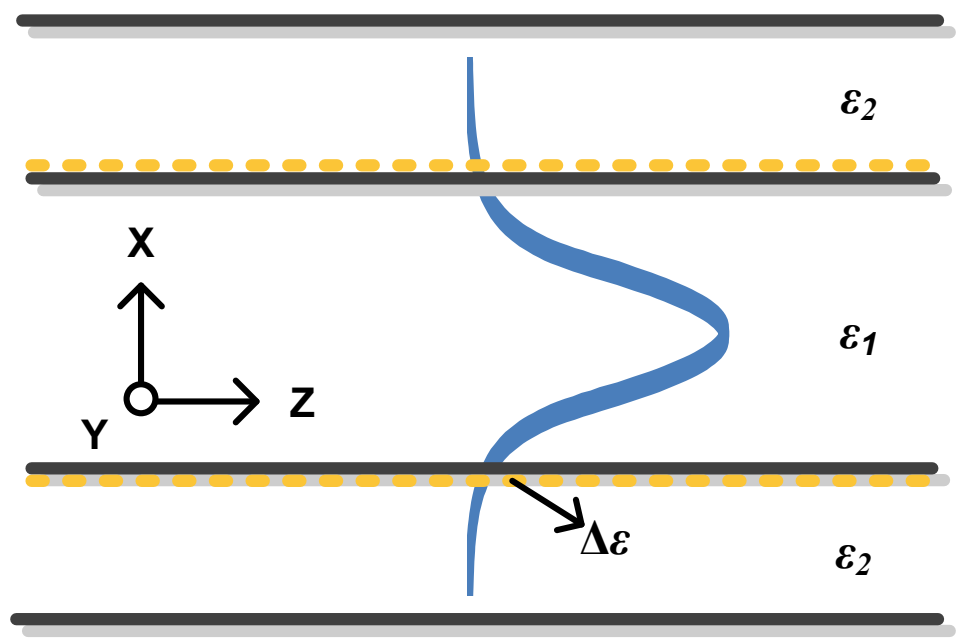

Fig. 2.2. Schematic drawing of a symmetric three-layer slab waveguide. An additional metal layer results in a permittivity perturbation that is different for TE and TM modes. 
When an additional metal layer applied in the optical waveguide, the change of the permittivity profile $(\Delta \varepsilon(x))$ leads to a small perturbation of the discrete eigenvalues $\Delta \beta^{2}$,

$$
\beta^{2}=\beta_{0}^{2}+\Delta \beta^{2}
$$

where $\beta_{0}$ is the initial propagation constant of a specific guided TE or TM mode without the perturbation. When the permittivity change $\Delta \varepsilon$ has an imaginary component, a mode attenuation arises that can be written as (Eq. (15) in Ref. [1]),

$$
\alpha=2 \operatorname{Im}\{\beta\} \approx \beta_{0}^{-1} \operatorname{Im}\left\{\Delta \beta^{2}\right\}
$$

It is important to note the attenuation coefficient is defined for the electric field intensity instead of its amplitude. The above derivation only includes first order effects of the perturbation (only the the eigenvalues change, not the eigenfunctions). Due to the correspondence between Eqs. (2.1) and (2.2) and the one-dimensional Schrödinger equation, the bound state perturbation theory indicates that the first order change in the eigenvalue $\Delta \beta^{2}$ induced by the small change of the permittivity $\Delta \varepsilon(x)$ can be expressed as the expectation value of the perturbation in the unperturbed state $[1,2]$,

$$
\begin{gathered}
\Delta \beta_{T E}^{2}=k_{0}^{2}\left\langle E_{y}, \Delta \varepsilon E_{y}\right\rangle_{T E} /\left\langle E_{y}, E_{y}\right\rangle_{T E} \\
\Delta \beta_{T M}^{2}=k_{0}^{2}\left\langle H_{y}, \Delta \varepsilon H_{y}\right\rangle_{T M} /\left\langle H_{y}, H_{y}\right\rangle_{T M}-\left\langle H_{y}, \frac{\partial}{\partial x} \frac{\Delta \varepsilon}{\varepsilon} \frac{\partial H_{y}}{\partial x}\right\rangle_{T M} /\left\langle H_{y}, H_{y}\right\rangle_{T M}
\end{gathered}
$$

where,

$$
\begin{aligned}
& \left\langle E_{y}, E_{y}\right\rangle_{T E}=\int_{-\infty}^{+\infty} E_{y}^{*}(x) E_{y}(x) d x \\
& \left\langle H_{y}, H_{y}\right\rangle_{T M}=\int_{-\infty}^{+\infty} H_{y}^{*}(x) \varepsilon(x)^{-1} H_{y}(x) d x
\end{aligned}
$$

Though $\Delta \beta_{T M}^{2}$ has an additional second term dependent on the permittivity perturbation of $\Delta \varepsilon(x)$ caused by the lossy metal layer, the second term can be eliminated for the step-index waveguides. 
Therefore, to first order the polarization-dependent spectral responses of the cladding modes in optical fibers caused by the addition of a thin gold coating, i.e. the wavelength shifts and amplitude changes of the mode resonances, are mainly determined by the perturbations of their propagation constants (i.e. complex effective indices) and such measurements can lead back to the properties of the perturbing films. However since the films at those thicknesses are not continuous, effective medium techniques must be applied.

\subsection{Effective medium approximations}

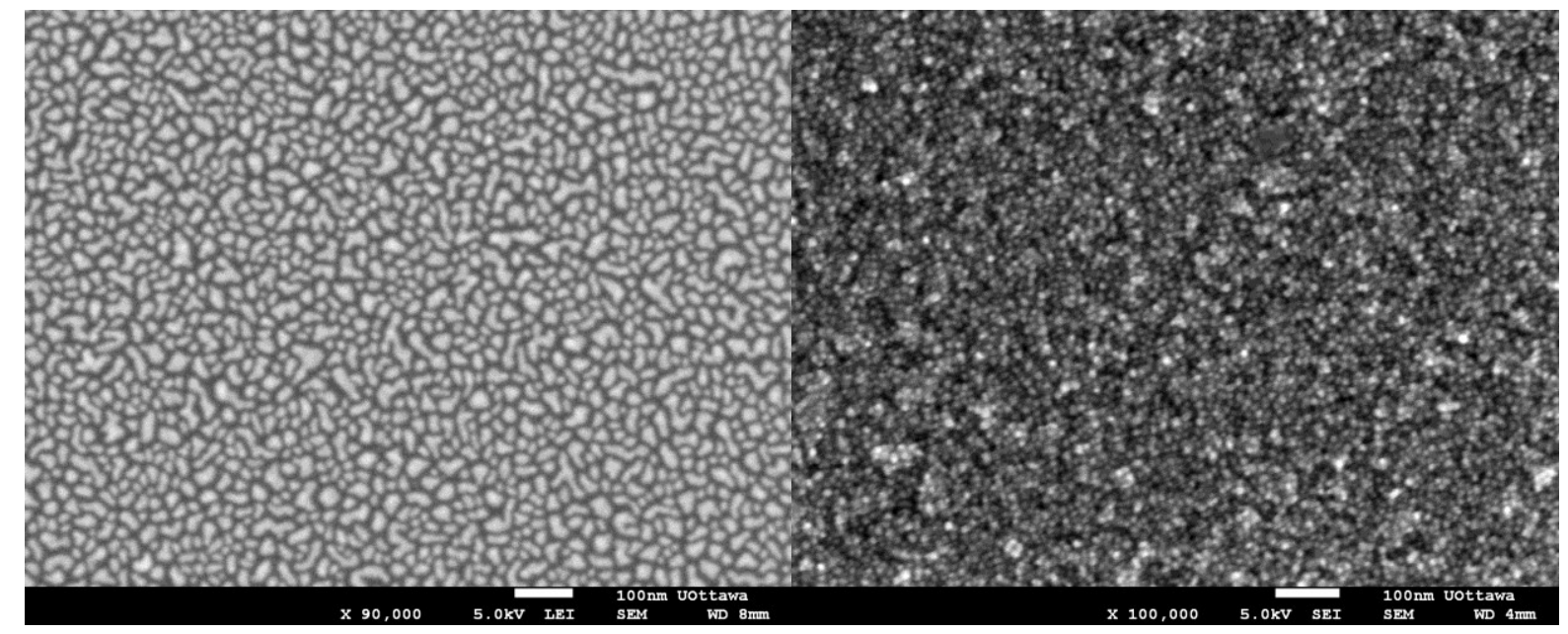

Fig. 2.3. SEM images of $5 \mathrm{~nm}$ thick gold evaporation film (left) and $50 \mathrm{~nm}$ thick gold CVD film (right).

In this section, we review two effective medium approximation (EMA) models that are frequently applied on simulating the optical properties of mixtures of two materials. For the ultrathin gold coatings on optical fiber surfaces, the films can be regarded as mixtures of gold inclusions and air dielectric background without considering the silica substrate (optical fiber) effect. From Fig. 2.3(a), it is very clear that the gold evaporation film with thicknesses around 5 $\mathrm{nm}$ is composed of gold nanoislands with random shapes and distributions. Even when the gold film grows up to a continuous state (such as the $\sim 50 \mathrm{~nm}$ gold CVD film shown in Fig. 2.3(b)), the top layer of the gold film should be still considered as a mixture layer of gold and air due to 
the significant surface roughness. Thus, the complex refractive indices (permittivity) of such gold coatings simulated from the spectral responses of the TFBG cladding mode resonances are in reality the effective refractive indices of gold-air mixtures. And this is the main reason that we use the EMA models to verify the obtained results from the TFBG data.

The effective permittivity of a mixture $\varepsilon_{\text {eff }}$ is defined as the relation between the average electric field $<\mathbf{E}>$ and flux density $<\mathbf{D}>$, expressed as [3],

$$
\langle D\rangle=\varepsilon_{\text {eff }}\langle E\rangle
$$

The average electric field and flux density can be expressed by weighing the internal $\mathbf{E}_{\boldsymbol{i}}$ and static external field $\mathbf{E}_{e}$ with the corresponding volume fractions,

$$
\begin{aligned}
& \langle D\rangle=f \varepsilon_{i} E_{i}+(1-f) \varepsilon_{e} E_{e} \\
& \langle E\rangle=f E_{i}+(1-f) E_{e}
\end{aligned}
$$

where $\varepsilon_{i}$ and $\varepsilon_{e}$ are the permittivities of the inclusions (gold) and surroundings (air), respectively, and $f$ is the volume filling factor of the gold material. Combining Eqs. (2.11), (2.12) and (2.13), the effective permittivity $\varepsilon_{e f f}$ can be written as

$$
\varepsilon_{\text {eff }}=\frac{f \varepsilon_{i} A+\varepsilon_{e}(1-f)}{f A+(1-f)}
$$

where $A=E_{i} / E_{e}$. For the simplest case of spherical dielectric inclusions, the field ratio $A$ can be derived from Maxwell electromagnetic equations and related boundary conditions as,

$$
A=\frac{3 \varepsilon_{e}}{\varepsilon_{i}+2 \varepsilon_{e}}
$$

It is important to note that Eq. (2.15) reflects another coefficient called polarisability, which relates the dipole moment of the individual inclusion to the external field. By substituting Eq. 
(2.15) into Eq. (2.14), we can obtain the well-known Maxwell-Garnett mixing formula for the spherical inclusion situation,

$$
\varepsilon_{e f f}=\varepsilon_{e}+3 f \varepsilon_{e} \frac{\varepsilon_{i}-\varepsilon_{e}}{\varepsilon_{i}+2 \varepsilon_{e}-f\left(\varepsilon_{i}-\varepsilon_{e}\right)}
$$

It can be seen that the effective permittivity is only determined by the individual permittivities and the volume fraction without size-dependence in this EMA model.
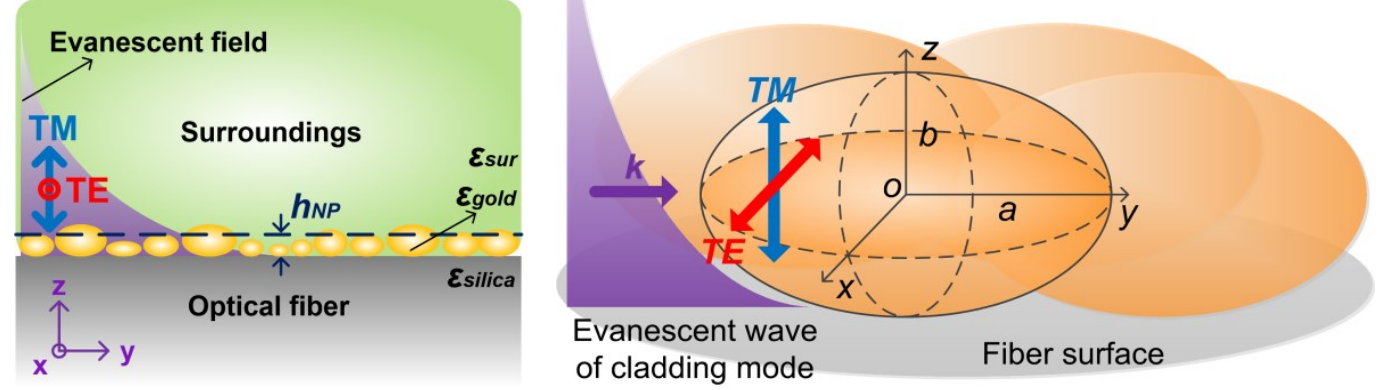

Fig. 2.4. 2D (left) and 3D (right) schematic diagram of spheroidal gold nanoparticles deposited on fiber surface (assuming that the fiber surface of small area $\left(\mu \mathrm{m}^{2}\right)$ is located in the $x y$ plane), interacting with evanescent field of polarized cladding mode. The gold spheroids are oblate so that the major axis $a$ and minor axis (polar axis) $b$ are parallel and perpendicular to the $x y$ plane, respectively.

Based on the microscopic images of the thin gold films taken with SEM and AFM, the film morphologies can be identified. For most of the gold films in this thesis, we usually regarded the films as a layer of oblate gold nanospheroids embedded within air dielectric matrix in the EMA models (as shown in Fig. 2.4). Due to the requirement for the quasi-static approximation that the size of the scatterers (gold nanospheroids) is considerably smaller than the wavelength of the operating field, the time-dependent electromagnetic field of the fiber-optic modes becomes identical to a static field at a given time instant in small regions of the gold films. And it means that the EMA models are independent to the wave-propagation properties of the 
mode fields. The two EMA formulas are used for modeling the oblate spheroidal inclusions in this thesis, one based on the generalized Maxwell-Garnett (M-G) formula [4] and the other on the generalized Bruggeman formula [5]. Thus, the generalized M-G and Bruggeman formulas can be respectively expressed as,

$$
\begin{aligned}
& \frac{\varepsilon_{e f f}-\varepsilon_{e}}{\varepsilon_{e}+N_{T M, T E}\left(\varepsilon_{e f f}-\varepsilon_{e}\right)}=f \frac{\varepsilon_{i}-\varepsilon_{e}}{\varepsilon_{e}+N_{T M, T E}\left(\varepsilon_{i}-\varepsilon_{e}\right)} \\
& (1-f) \frac{\varepsilon_{e f f}-\varepsilon_{e}}{\varepsilon_{e f f}+N_{T M, T E}\left(\varepsilon_{e}-\varepsilon_{e f f}\right)}=f \frac{\varepsilon_{i}-\varepsilon_{e f f}}{\varepsilon_{e f f}+N_{T M, T E}\left(\varepsilon_{i}-\varepsilon_{e f f}\right)}
\end{aligned}
$$

where $N_{T M, T E}$ is the depolarization factor for TM or TE-polarization. For the case of spherical inclusions, $N_{T M, T E}$ is equal to $1 / 3$. While for the oblate gold spheroids, the depolarization factors of the TM- and TE-polarizations can be defined as a function of the aspect ratio $x$ of the oblate spheroid [3],

$$
\begin{aligned}
& N_{T M}=\frac{1+e^{2}}{e^{3}}\left(e-\tan ^{-1} e\right) \\
& N_{T E}=\left(1-N_{T M}\right) / 2
\end{aligned}
$$

where $e=\sqrt{a^{2} / b^{2}-1}, a$ and $b$ are the length of the major and minor axes, respectively. With Eqs. (2.11) and (2.12), the complex refractive indices (permittivities) of the effective medium coating for the out-of-plane (TM) and in-plane (TE) directions can be calculated.

\subsection{OptiGrating (by Optiwave) and FIMMWAVE (by Photon Design)}

The OptiGrating [6] software was using for calculating the imaginary part of effective index of cladding mode, based on the amplitude attenuation of the cladding mode extracted from the gold-coated TFBG. By adjusting the modulation amplitude of the refractive index of grating and the overlap integral between the core mode and cladding mode, the amplitude of the selected cladding mode resonance of the bare TFBG can be simulated in OptiGrating. Then, the 
amplitude attenuation of the cladding mode induced by the gold coating can be introduced into the model by adding an imaginary part to the effective index of the cladding mode. Fig. 2.5 shows the spectral evolution (top) of an attenuating cladding mode with increasing imaginary part of the effective index, and the corresponding fitting curve (bottom) of the resonance amplitude versus the imaginary part of the mode propagation constant. It is very obvious that the cladding mode profile becomes shallower and broader with an increased imaginary part.
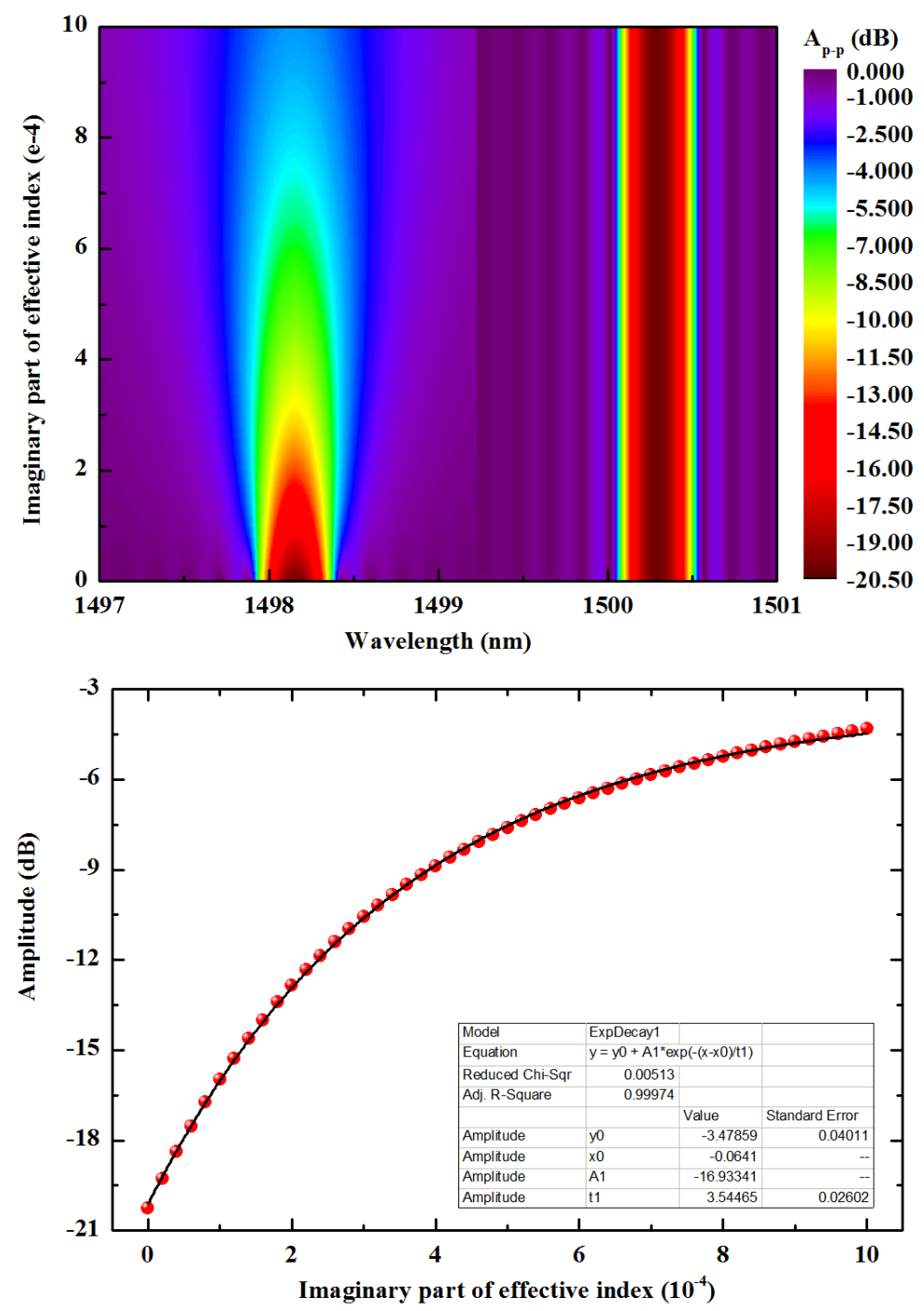

Fig. 2.5. Spectral evolution (top) of an attenuating cladding mode with increasing imaginary part (near $1498 \mathrm{~nm})$, compared with a cladding mode without attenuation $(1500 \mathrm{~nm})$. Fitting curve (bottom) of the cladding mode resonance amplitude versus the corresponding imaginary part. 
The FIMMWAVE [7] software was used for supporting the calculation of the real part of effective index of cladding modes and for retrieving the effective refractive indices of the gold coatings, based on the measured effective indices of the cladding modes of the gold-coated TFBGs. From the phase matching condition between core mode and cladding mode in a TFBG, the real part of the effective index can be expressed as

$$
N_{e f f}^{c l}\left(\lambda_{c l}\right)=\frac{2 N_{e f f}^{c o}\left(\lambda_{c o}\right) * \lambda_{c l} * \cos \theta}{\lambda_{c o}}-N_{e f f}^{c o}\left(\lambda_{c l}\right)
$$

where $\lambda_{c o}$ and $\lambda_{c l}$ are the wavelengths of the core mode and the cladding mode resonances, respectively, and $N_{e f f}^{c o}\left(\lambda_{c o}\right), N_{e f f}^{c o}\left(\lambda_{c l}\right)$ and $N_{e f f}^{c l}\left(\lambda_{c l}\right)$ are the effective indices of the core mode at $\lambda_{c o}$ and $\lambda_{c l}$, and the cladding mode at $\lambda_{c l}$, respectively. $N_{e f f}^{c o}\left(\lambda_{c o}\right)$ and $N_{e f f}^{c o}\left(\lambda_{c l}\right)$ are calculated by adjusting the operating wavelength in FIMMWAVE and solving for the single mode of the core. The mode solver in FIMMWAVE is based on vectorial finite differences, where only the radial dimension is discretized. The aim of the simulation is to find the complex refractive index of the thin gold film $\left(n_{f}-i k_{f}\right)$ that reproduces the spectral shifts extracted from TFBG spectrum, for each film thickness and for each mode polarization. Separate calculations for TE and TM modes allow the determination of any anisotropy (birefringence in $n_{f}$ or dichroism in $k_{f}$ ) in the deposited coatings.

\subsection{Deposition methods of gold coatings}

Due to the cylindrical structure of optical fibers, the chemical vapor deposition (CVD) technique was used to deposit conformal gold coating around the optical fiber surface [8]. In this thesis, two kinds of gold CVD precursors were used, which were gold (I) iminopyrrolidinate ([Au( $\mathrm{Me}_{2}-$ $\left.\left.\left.{ }^{t} \mathrm{Bu}-\mathrm{ip}\right)\right]_{2}\right)$ and gold (I) guanidinate $\left(\left[\mathrm{Au}(\mathrm{NiPr})_{2} \mathrm{CNMe}_{2}\right]_{2}\right)$. By simply heating the gold precursors to specific temperatures $\left(350{ }^{\circ} \mathrm{C}\right.$ for $\left[\mathrm{Au}\left(\mathrm{Me}_{2}-{ }^{\mathrm{t}} \mathrm{Bu} \text {-ip) }\right]_{2}\right.$ and $250{ }^{\circ} \mathrm{C}$ for $\left.\left[\mathrm{Au}(\mathrm{NiPr})_{2} \mathrm{CNMe}_{2}\right]_{2}\right)$ 
under a base pressure of 5 mTorr, the precursors vaporize and decompose in the deposition chamber. Then the gold nanoparticles begin to nucleate on all exposed surfaces including the optical fiber cladding.

Moreover, another gold physical vapor deposition (PVD) method (gold evaporation) was also applied for coating the gold film on optical fibers (TFBGs), because of the anomalous optical properties of the gold CVD films [9]: different from the gold CVD, the gold evaporation is highly directional and cannot coat uniform films on optical fiber surfaces. So in order to have a relatively uniform gold coating around the fiber circumference by PVD, two gold deposition steps were conducted consecutively with the fiber being rotated by exactly $180^{\circ}$ between the two deposition runs using a custom designed fiber holding fixture. It is important to note that the orientation of the grating planes of the TFBG can be determined with high precision by observing the scattering patterns of visible light injected in the fiber, thereby allowing the alignment of the fiber grating plane tilt with the two PVD deposition steps. This alignment method is decribed in further detail in chapter 6.

\subsection{Experimental setup of the optical measurements}

(a)

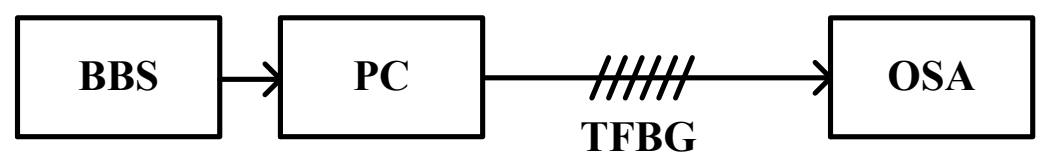

(b)

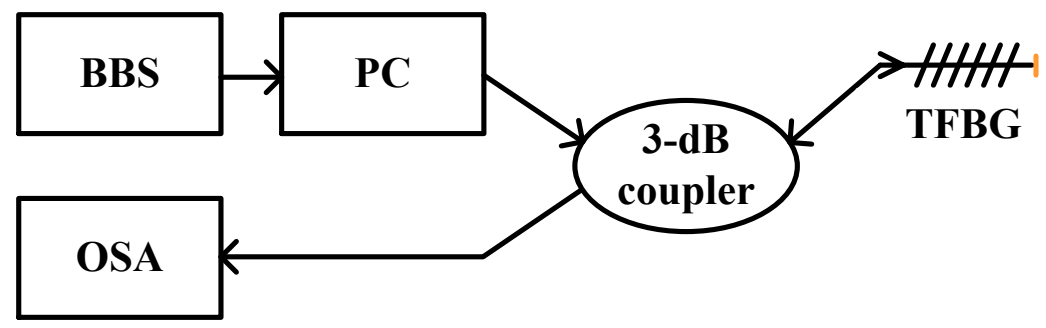

Fig. 2.6. Schematic diagram of experimental setups for TFBG transmission (a) and reflection (b) spectra measurements. The measurement setup is dependent on the style of TFBG probe, which is based on the 
gold deposition methods. Basically, the reflective TFBG probe with a gold mirror on the end is used in gold CVD; while the normal TFBG sensing head is used in gold evaporation chamber.

The TFBG transmission and reflection measurement systems are shown in Figs. 2.6 (a) and (b), respectively. The fiber-coupled broadband source (BBS) (JDSU BBS1560) launches light from 1520 to $1620 \mathrm{~nm}$ in the same type of single mode telecommunication fiber is used for the TFBG. Even though the fibers are not of the polarization maintaining type, a polarization controller (PC) (JDSU PR2000) that contains one polarizer, a half-waveplate and a quarter-waveplate, can be used to generate a state of polarization that will evolve to a linear state either parallel (ppolarized, or TM) or perpendicular (s-polarized or TE) at the grating. By rotating the halfwaveplate and the quarter-waveplate in turn, the TFBG cladding resonances can be adjusted to the largest amplitudes that indicate the correct TM- or TE-polarization state. And the two polarization states can be switched by rotating the polarizer by 90 degree. As long as the fiber paths are not moved during the experiments, the polarization state remains stable. As indicated earlier, when the polarization state of incident light at the TFBG contains only TE- or TMpolarized light, the electrical field of the excited high-order cladding modes are predominantly azimuthal or radial at the cladding boundary respectively and the associated TFBG resonances occur at different wavelengths. For the reflective TFBG measurement system (Fig. 2.6(b)), the light goes through the grating twice and returns towards the light source in the same fiber; a 50:50 fiber coupler picks off part of the reflected light to be measured by an optical spectrum analyzer (OSA) (ANDO AQ6317B) with a nominal resolution of $0.01 \mathrm{~nm}$. Fig. 2.7 shows parts of a 10 degree tilt TFBG transmission spectra measured under TE-polarized, TM-polarized and un-polarized incident light, with the maximum transmission normalized to the same level. The correct orientations of the PC provide TM- and TE-polarized cladding modes resonances with 
the maximum amplitudes, while un-polarized light results in pairs of resonances with each approximately coupling only half the light (3 dB deep).

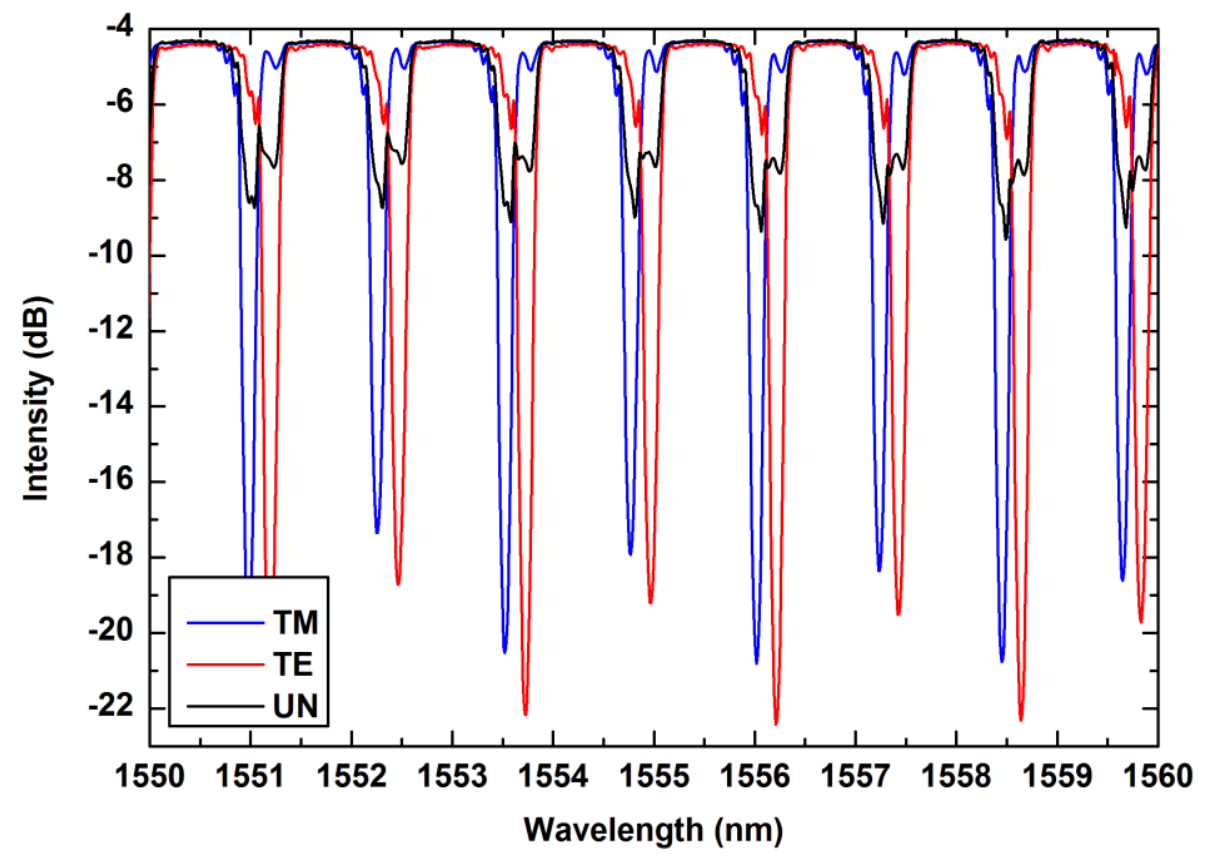

Fig. 2.7. Measured $10^{\circ}$ TFBG spectra under TE-polarized, TM-polarized and un-polarized incident lights. The Bragg resonance is around $1610 \mathrm{~nm}$.

\subsection{References}

1. K. O. Hill, A. Watanabe, and J. G. Chambers, "Evanescent-wave interactions in an optical fiber wave-guiding structure," Appl. Opt. 11(9), 1952-1959 (1972).

2. W. H. Weber, S. L. McCarthy, and G. W. Ford, "Perturbation theory applied to gain or loss in an optical waveguide,” Appl. Opt. 13(4), 715-716 (1974).

3. A. Sihvola, Electromagnetic Mixing Formulas and Applications (The Institution of Electrical Engineers, 1999), Chap. 3-4.

4. J. C. Maxwell Garnett, "Colours in metal glasses and in metallic films," Phil. Trans. R. Soc. Lond. A 203, 385-420 (1904).

5. D. A. G. Bruggeman, "Berechnung verschiedener physikalischer Konstanten von heterogenen Substanzen. I. Dielektrizitätskonstanten und Leitfähigkeiten der Mischkörper aus isotropen Substanzen," Ann. Phys. 416(7), 636-664 (1935).

6. http://optiwave.com/category/products/component-design/optigrating/

7. http://www.photond.com/products/fimmwave.htm 
8. D. J. Mandia, W. Zhou, J. Albert, and S. T. Barry, "CVD on optical fibers: tilted fiber Bragg gratings as real-time sensing platform," Chem. Vap. Deposition 20, 1-17 (2014). DOI: $10.1002 /$ cvde. 201400059

9. W. Zhou, D. J. Mandia, M. B. E. Griffiths, S. T. Barry, and J. Albert, "Anomalous refractive index of ultrathin gold nanoparticle film coated on tilted fiber Bragg grating," Proc. of SPIE 9157, 91573Y (2014). 


\section{Chapter 3: Polarization-Dependent Properties of the}

\section{Cladding Modes of a Single Mode Fiber Covered with Gold}

\section{Nanoparticles $^{1}$}

The properties of the high order cladding modes of standard optical fibers are measured in real-time during the deposition of gold nanoparticle layers by chemical vapour deposition (CVD). Using a tilted fiber Bragg grating (TFBG), the resonance wavelength and peak-to-peak amplitude of a radially polarized cladding mode resonance located $51 \mathrm{~nm}$ away from the core mode reflection resonance shift by $0.17 \mathrm{~nm}$ and $13.54 \mathrm{~dB}$ respectively during the formation of a $\sim 200 \mathrm{~nm}$ thick layer. For the

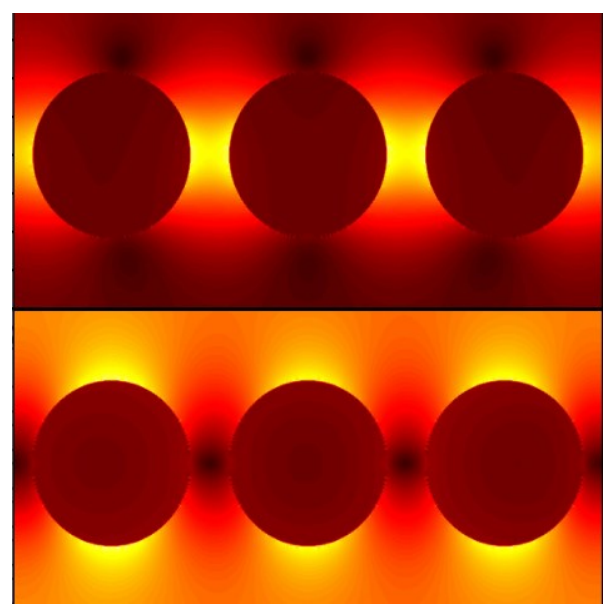
spectrally adjacent azimuthally polarized resonance, the corresponding shifts are $0.45 \mathrm{~nm}$ and $16.34 \mathrm{~dB}$. In both cases, the amplitudes of the resonance go through a pronounced minimum of about $5 \mathrm{~dB}$ for thickness between 80 and $100 \mathrm{~nm}$ and at the same time the wavelengths shift discontinuously. These effects are discussed in terms of the evolving metallic boundary conditions perceived by the cladding modes as the nanoparticles grow. Scanning Electron Micrographs and observations of cladding mode light scattering by nanoparticle layers of various thicknesses reveal a strong correlation between the TFBG polarized transmission spectra, the grain size and fill factor of the nanoparticles, and the scattering efficiency. This allows the preparation of gold nanoparticle layers that strongly discriminate between radially and azimuthally polarized cladding mode evanescent fields, with important consequences in the plasmonic properties of these layers.

\footnotetext{
${ }^{1}$ Reproduced with Permission from "W. Zhou, D. J. Mandia, M. B.E. Griffiths, A. Bialiayeu, Y. Zhang, P. G. Gordon, S. T. Barry, and J. Albert, 'Polarization-dependent properties of the cladding modes of a single mode fiber covered with gold nanoparticles, ' Optics Express 21(1), 245-255 (Jan. 2013). ” Copyright 2013, Optical Society of America. Author contributions: J.A. conceived the basic idea of a TFBG-based in situ monitoring system for gold CVD process. J.A. planned, supervised and supported the experimental implementation. W.Z. and Y.Z. fabricated the TFBG sensors. W.Z. built the experimental setup, and performed the experimental data processing. D.J.M., M.B.E.G., and P.G.G. fabricated the gold CVD precursors, and performed the gold CVDs. P.G.G. performed the SEM imaging. A.B. performed the numerical simulations. W.Z., D.J.M., A.B., and J.A. wrote the manuscript. The project was organized and coordinated by J.A. and S.T.B.
} 


\subsection{Introduction}

The interaction between propagating light waves and nanometer-sized gold particles presents many interesting features that have been studied in several contexts $[1,2]$. It is therefore expected that depositing gold nanoparticles on the cladding of an optical fiber will have an impact on the cladding-guided modes of such fibers and may lead to new applications in sensing, all optical switching, and nonlinear optics. Tilted fiber Bragg gratings (TFBGs) are ideally suited for such studies because of their capability to excite a number of cladding modes whose properties can be probed very precisely by measuring the wideband spectral response of the grating transmission [3-4]. Furthermore, due to the breakup of the circular symmetry caused by the tilted grating planes, the polarization state of the incident core mode controls the orientation and polarization of the excited cladding modes at the cladding boundary [4]. This is of utmost importance for metal coatings as the boundary conditions for metal-dielectric interfaces depend very strongly on the polarization state of the light. For example, cladding modes with radial polarization at the cladding boundary can be excited selectively and be used to couple light into surface plasmon waves on metal coated fibers. A gold-coated TFBG refractometer based on Surface Plasmon Resonance (SPR) was demonstrated using this technique [5], and shown to yield a great enhancement in the minimum surrounding refractive index (SRI) detection level [6], compared to non-TFBG fiber SPR devices $[7,8]$. In all these fiber-based SPR configurations the quality and uniformity of the (typically) $50 \mathrm{~nm}$ thick metal layer is critical but also difficult to control; especially to make it uniform around the circumference of the fiber. The gold coatings in the papers mentioned above were fabricated by sputtering or evaporation techniques, which require fiber rotations to obtain approximately uniform films. Better uniformity gold films are expected from conformal coating approaches such as electroless plating and chemical vapour deposition 
(CVD) which have been demonstrated for gold and copper respectively $[9,10]$. Recently, a new precursor for gold CVD was developed [11] and the present work deals with an investigation of the effect of this new CVD coating process on the properties of the cladding modes of such coated fibers. As shown in Fig. 3.1, we used single-ended $10^{\circ}$ tilt TFBGs inserted in the CVD process chamber and monitored their transmission spectrum from the input side by using the reflection from a gold mirror deposited on the fiber end. This avoids having to loop back the fiber inside the chamber. The wavelengths and amplitudes of resonances associated with cladding modes of different polarization states were obtained throughout the CVD process. The observations obtained from inside the fiber using the TFBG resonances were compared with scanning electron microscope images of the films at various stages of deposition, and with infrared camera images of the light scattered out of the fiber by the nanoparticles. The results indicate three different regimes according to the density and thickness of the CVD gold nanoparticle coatings. In particular, during the coating process we observed a well defined transition between isolated nanoparticles and a semi-continuous film that has a strong impact on the optical properties of the optical fiber cladding modes.

\section{Gold nanoparticles}

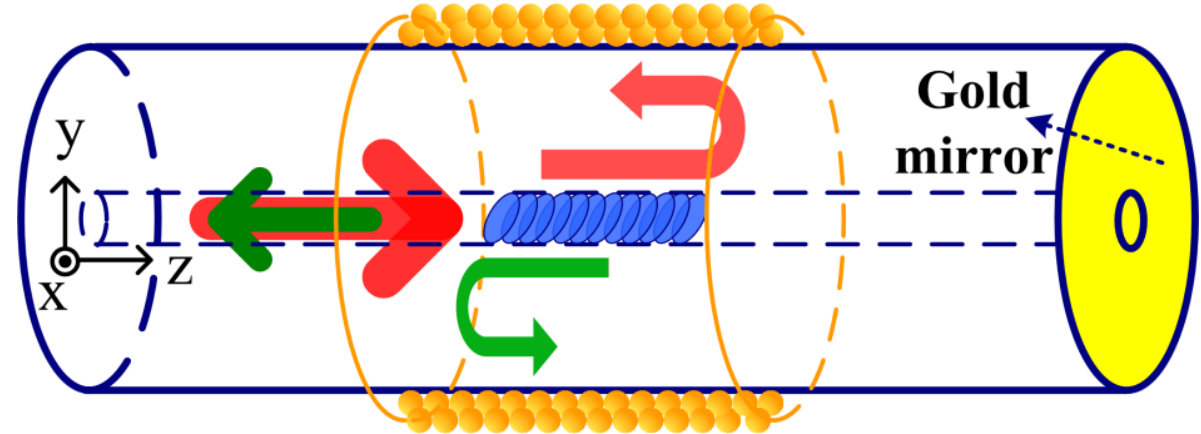

Fig. 3.1. Schematic diagram of TFBG coated by gold nanoparticles (the arrows show how the incident core guided light (red) goes through the grating twice, each time coupling light to cladding modes). The light remaining in the core (straight green arrow) returns to the interrogation system. 


\subsection{Principle and sensor fabrication}

In order to clarify how nanoscale metal coatings influence cladding mode resonances, a brief review of TFBG properties is required before the description of the experiments. A periodical refractive index perturbation with tilted grating planes along the core axis couples the forwardpropagating core mode to a number of backward-propagating cladding modes in addition to a backward-propagating core mode (the "Bragg" mode). The wavelength of the resonance of the Bragg mode, $\lambda_{\text {Bragg }}$ and of the $i$ th order cladding mode resonance $\lambda_{\text {cladding }}^{i}$ in the transmission spectrum of TFBG can be expressed as [12]

$$
\begin{aligned}
& \lambda_{\text {Bragg }}=2 N e f f_{\text {Bragg }} \Lambda / \cos \theta \\
& \lambda_{\text {cladding }}^{i}=\left(N e f f_{\text {Bragg }}^{i}+N e f f_{\text {cladding }}^{i}\right) \Lambda / \cos \theta
\end{aligned}
$$

where Neff $f_{\text {Bragg }}$ is the effective index of core mode at the Bragg wavelength, $\Lambda$ is the grating period, $\theta$ is the tilt angle of the grating planes, Neff $f_{\text {Bragg }}^{i}$ and $N e f f_{\text {cladding }}^{i}$ are the effective indices of the core mode and $i$ th cladding mode at the wavelength of the $i$ th cladding resonance. The guiding properties of these cladding modes and hence of Neff $_{\text {cladding }}$ depend on the permittivity of the medium in which the cladding is located. Therefore we expect the wavelengths of the cladding mode resonances to shift during the deposition of gold nanoparticles on the fiber. The amplitudes of these cladding resonances would also change under the influence of the imaginary part of the complex refractive index of gold (as shown for LPGs in [13]) and of the loss due to scattering by the nonuniform layer of nanoparticles on the surface of the TFBG.

We further expect these effects to depend strongly on the polarization state of the cladding modes at the cladding boundary. The tilted grating planes break the azimuthal symmetry of the fiber and two orthogonal polarization states of the electrical field input light can 
be defined relative to the tilt plane: S-polarized light with the electrical field perpendicular to $y-z$ plane and P-polarized light with the electrical field parallel to y-z plane (as shown in Fig. 3.1). In previous reports, we showed that when the polarization state of the light at the TFBG contains only S- or P-polarized light (two extreme cases), the electrical field of the excited high order cladding modes also has radically different polarization properties [5,14]. Simulations obtained with a finite difference mode solver [15], and shown in Fig. 3.2, indicate that S-polarized light can only couple into high order cladding modes that have their electrical field tangential to the cladding boundary, while P-polarized light excites cladding modes with predominantly radial electrical fields. This difference is highlighted in Fig. 3.3, where two transmission spectra of the same grating under S- and P-polarized light interrogation (measured in air without coating) show clearly different sets of resonances that occur in closely spaced pairs. Similarly to TFBGs with uniform thin metal layers, we use this polarization selectivity to obtain additional information about the gold nanoparticle coating as it grows.
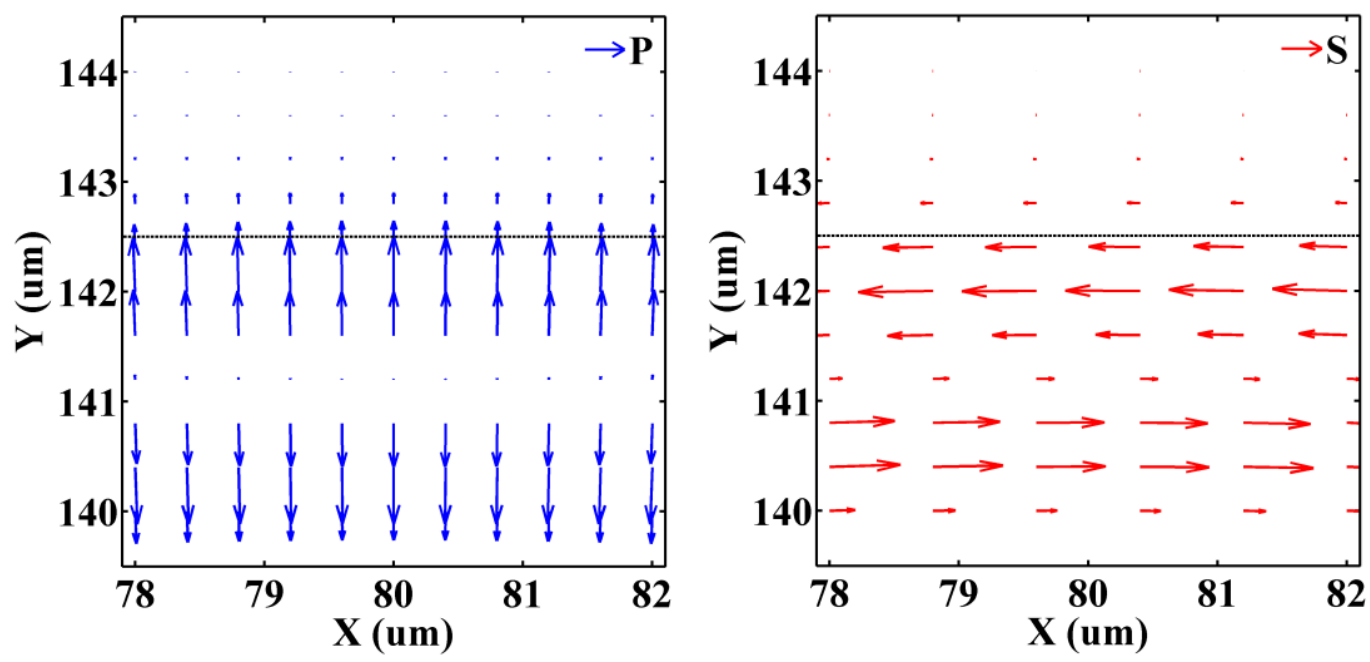

Fig. 3.2. Simulated electrical fields of a high-order cladding mode excited by P- (left) and S-(right) polarized light. The figures represent a small area of 4 by $4 \mu \mathrm{m}^{2}$ close to cladding boundary (indicated by a horizontal line at $\mathrm{Y}=142.5 \mu \mathrm{m})$. All results were calculated with FIMMWAVE. 


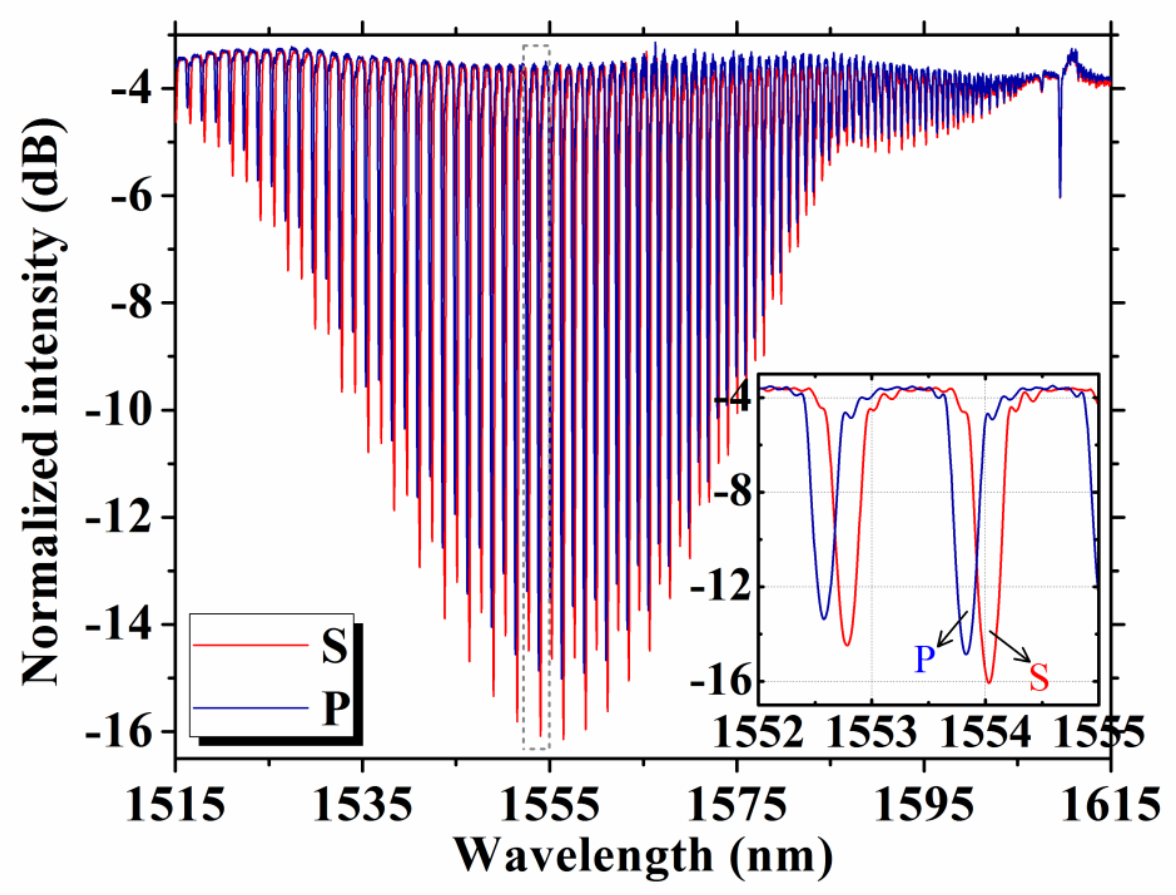

Fig. 3.3. Experimental transmission spectra of $10^{\circ} \mathrm{TFBG}$ with S- and P-polarized input light in air, inset: detailed spectra from 1550 to $1556 \mathrm{~nm}$.

The TFBGs were written in hydrogen-loaded photosensitive CORNING SMF-28 fiber with a pulsed $\mathrm{KrF}$ excimer laser using the phase-mask method. The Bragg wavelength is around $1610 \mathrm{~nm}$ so that important cladding modes are observed in the middle of the C-band (near 1550 $\mathrm{nm})$. The length of TFBG is only $4 \mathrm{~mm}$ long in order to minimize the impact of eventual coating thickness non-uniformities on the grating response. A tilt angle of $10^{\circ}$ leads to a large number of strong, high order cladding mode resonances that have larger evanescent field penetration outside the cladding. In order to have a single entry port in the furnace used for the CVD process and to avoid bending the fiber, a reflective sensing configuration of TFBG was implemented by cleaving the fiber $1.5 \mathrm{~cm}$ downstream from the TFBG and coating the end with a sputtered gold mirror (Fig. 3.1). This configuration further enhances the response of the TFBG since coreguided light goes through the grating twice. The gold mirror at the downstream end was prepared separately by conventional sputtering, and its thickness (several microns) is such that the 
addition of more gold on the fiber end (during the nanoparticle deposition process) has no effect on its reflectance. There is unavoidable growth on the fiber end but it has no influence on the properties of the cladding modes at the location of the grating (a few cm away).

\subsection{Experiment and results}

Figure 3.4 illustrates the experimental setup of the gold CVD system and the in situ spectral measurement system. The fiber was fixed on a metal boat and the end containing the TFBG was inserted into a vial filled with a single-source gold precursor ([Au(NiPr$\left.\left.)_{2} \mathrm{CNMe}_{2}\right]_{2}\right)[11]$.

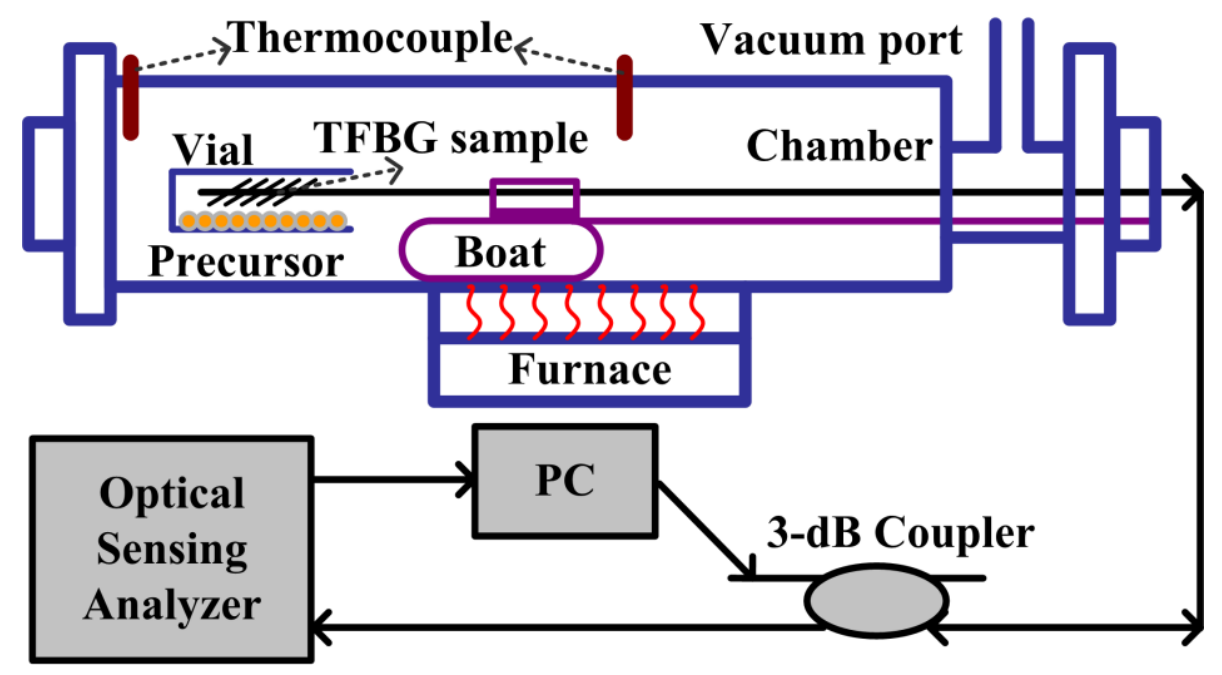

Fig. 3.4. Schematic diagram of gold CVD system and spectral monitoring setup. The optical sensing analyzer includes a scanning laser source with a wavelength range from 1520 to $1570 \mathrm{~nm}$ and a synchronized photodetector.

The fiber and vial were located in a deposition chamber made of stainless steel, the chamber itself being located within a heating furnace. At the beginning of the experiment the chamber was pumped down to a base pressure of 30 mTorr and then the furnace temperature ramp $\left(11^{\circ} \mathrm{C} / \mathrm{min}\right)$ program was set to $255^{\circ} \mathrm{C}$. Since the reaction chamber is situated far from the main thermocouple, temperature measurements were collected on a second thermocouple fitted further down the furnace tube to account for any thermal lag. As the temperature increases, it 
reaches the vaporization and decomposition point of the highly volatile precursor $\left(\sim 220^{\circ} \mathrm{C}\right)$ and the gold nanoparticles begin to nucleate on all exposed surfaces including the cladding of the TFBG. EDS analysis performed on the fibers and planar samples with this gold compound showed no significant or detectable impurities (only signals for $\mathrm{Si}, \mathrm{O}$, and $\mathrm{Au}$ ). The process selfterminates when the precursor has fully reacted and the furnace is then allowed to cool down. The whole process takes about 10 minutes and the onset of the deposition itself occurs after approximately 8 minutes, as corroborated by changes in the TFBG response. With this methodology, the final thickness of the gold nanoparticle coating on the fiber is determined by the amount of precursor in the vial. For instance, the film thickness from a $30 \mathrm{mg}$ sample of precursor was typically $\sim 200 \mathrm{~nm}$. In other experiments wherein the mass was varied, a linear relationship was found with respect to the film thickness, and a nominal growth rate of $5 \mathrm{~nm} / \mathrm{s}$ (starting from onset of deposition) was calculated from ex-situ thickness calculations using WSXM 5.0 image processing software [16]. Finally, the impact of the strong temperature changes on the spectral positions of the resonances was removed from the data analysis by recording the wavelength shift of the Bragg mode and subtracting it from the shifts of the cladding modes. The Bragg mode is well isolated from the cladding boundary (therefore from any effect resulting from the deposition) and it has been verified that all cladding mode resonances have the same intrinsic temperature dependence as the Bragg mode [12]. It must be noted that this deposition configuration yields coatings that vary in thickness as a function of distance from the precursor source. We usually work with longer TFBGs (between 10 and 20 $\mathrm{mm}$ long) in order to have narrower resonances to enhance the Q-factor and thus the minimum detectable level of sensors, but if the coating thickness varies over this length, the net effect is a chirp of the grating properties and widening of resonances. Also, we need only to deposit the 
particles on the fiber sections where the grating is located, because these are the only locations where light is emitted into the cladding.

Before the deposition, the polarization of the incident light was adjusted to the exact S- or P-polarization state using an electronic polarization controller (PC) (JDS Uniphase) that contains one polarizer, a half-waveplate and a quarter-waveplate. This combination allows the preparation of arbitrary polarization states at the fiber input, which can compensate for any change of polarization state induced by fiber loops and twists in the optical path leading to the TFBG. The optimum launch polarization is simply determined by observing the transmission spectrum and maximizing either one of the two sets of transmission resonances. The spectral evolution was recorded continually during the deposition by an optical sensing analyzer (Micron Optics Si720) with a measurement frequency of $5 \mathrm{~Hz}$. Even though $\mathrm{S}$ and P polarized spectra were obtained during separate experiments, it was verified that the results were reproducible for identical process parameters.

Figure 3.5 shows the evolution of the TFBG reflective transmission ranging from 1550 to $1560 \mathrm{~nm}$ (where the cladding modes have the largest amplitudes for $10^{\circ} \mathrm{TFBG}$ ) with S- and Ppolarized light. The origin of the time scale in Fig. 3.5 is determined by the moment when the precursor begins to evaporate, as indicated by a small pressure rise in the chamber (near 8 minutes into the process, as mentioned above). Basically, both the S- and P-polarized spectra go through a similar evolution in that all cladding modes become much attenuated at first, and then re-grow back to a shape very similar to the original one measured in air. There are subtle differences however: the initial attenuation occurs faster and lasts longer for P-polarized light, but most importantly the re-appearance of the resonances as the thickness increases is accompanied by a strong wavelength shift for S-polarized light but hardly any at all for the P- 
polarized case. This is further clarified in quantitative terms by plotting the amplitude and wavelength of one pair of resonances near $1559 \mathrm{~nm}$ as shown in Fig. 3.6.
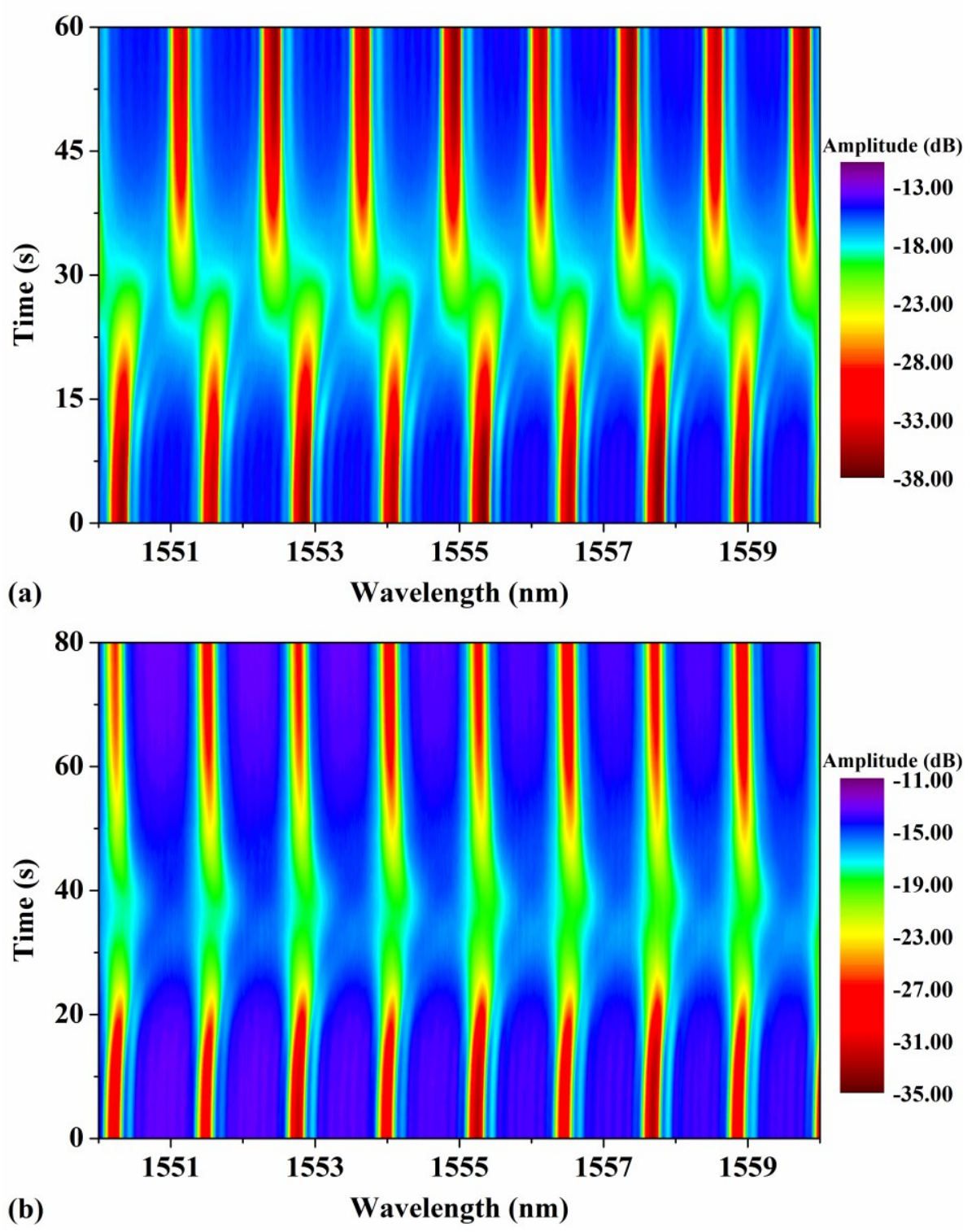

Fig. 3.5. TFBG spectral evolutions of during gold nanoparticles deposition under S- (a) and P-polarized (b) light (the color scale represent the amplitude of the resonances). Spectra are acquired every second and temperature-corrected with reference to the Bragg mode shifts.

The results indicate that the amplitudes of S-resonances decrease by $15 \mathrm{~dB}$ in the first 25 $\mathrm{s}$ of deposition and recover completely after $60 \mathrm{~s}$. Still in the first 25 seconds, the resonance 
wavelength red-shifts by about $\sim 0.08 \mathrm{~nm}$. Most importantly, near the minimum amplitude point the wavelength discontinuously blue-shifts by $\sim 0.4 \mathrm{~nm}$ and stays relatively constant for the remainder of the deposition, as the resonance gradually regains its full amplitude. The Presonances also decrease in amplitude but by a slightly smaller amount and about 10 seconds later than the minimum of the S-resonance. The most significant difference however is that the wavelength shift of P-resonances is completely antagonistic to that of the S-resonance: it first increases slowly by about $0.06 \mathrm{~nm}$, then decreases by $0.05 \mathrm{~nm}$ and when the amplitude minimum is reached near the 35 second point, jumps discontinuously upward by $0.14 \mathrm{~nm}$. The net result is that there is an interchange of the S- and P-resonances during the deposition. This confirms our previous finding, obtained for continuous, $50 \mathrm{~nm}$-thick sputtered gold films [6] that the S- and Presonances were interchanged between air-clad and metal-clad fibers.

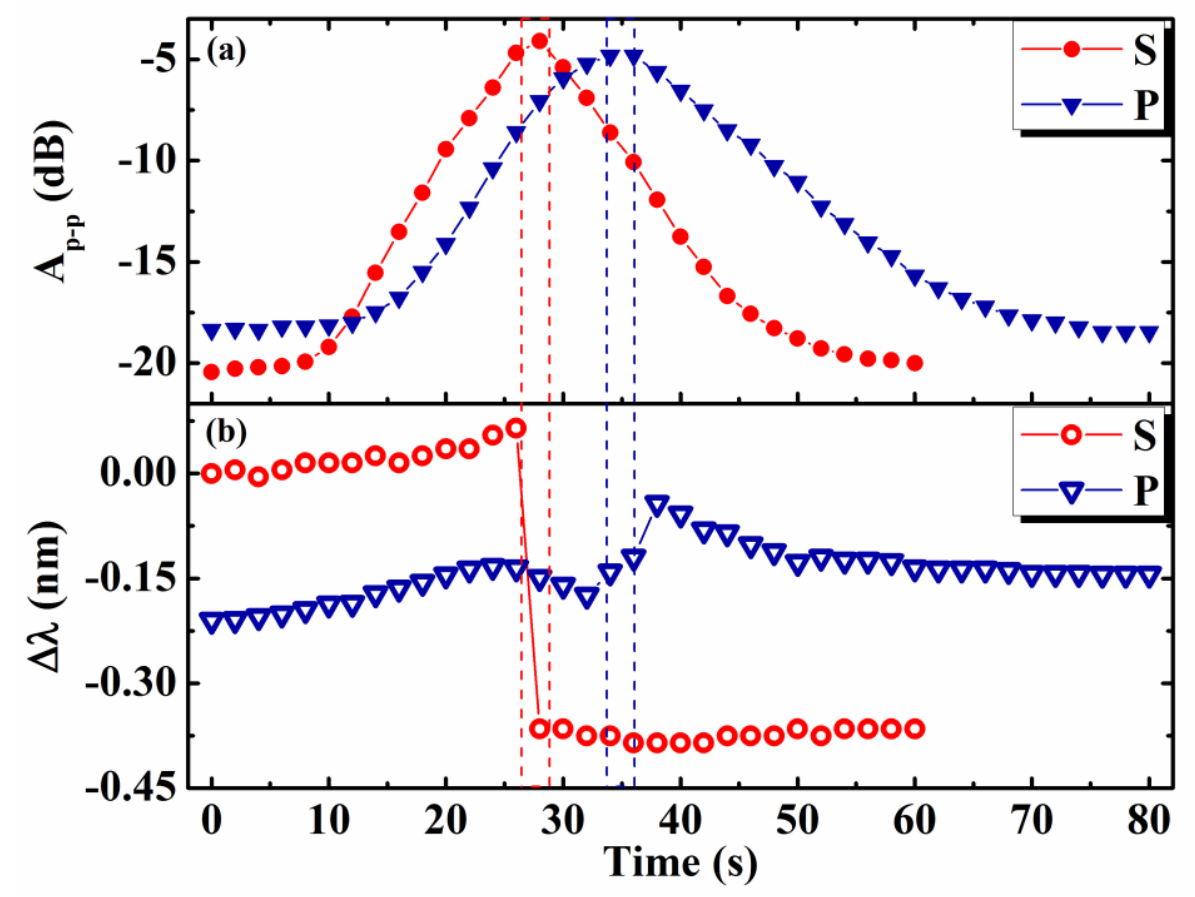

Fig. 3.6. Normalized amplitude (a) and wavelength (b) evolutions versus time of gold deposition under Sand P-polarized light. The vertical dashed lines bound the minimum amplitudes for each polarization, highlighting the simultaneous occurrence of the corresponding wavelength change discontinuities. 


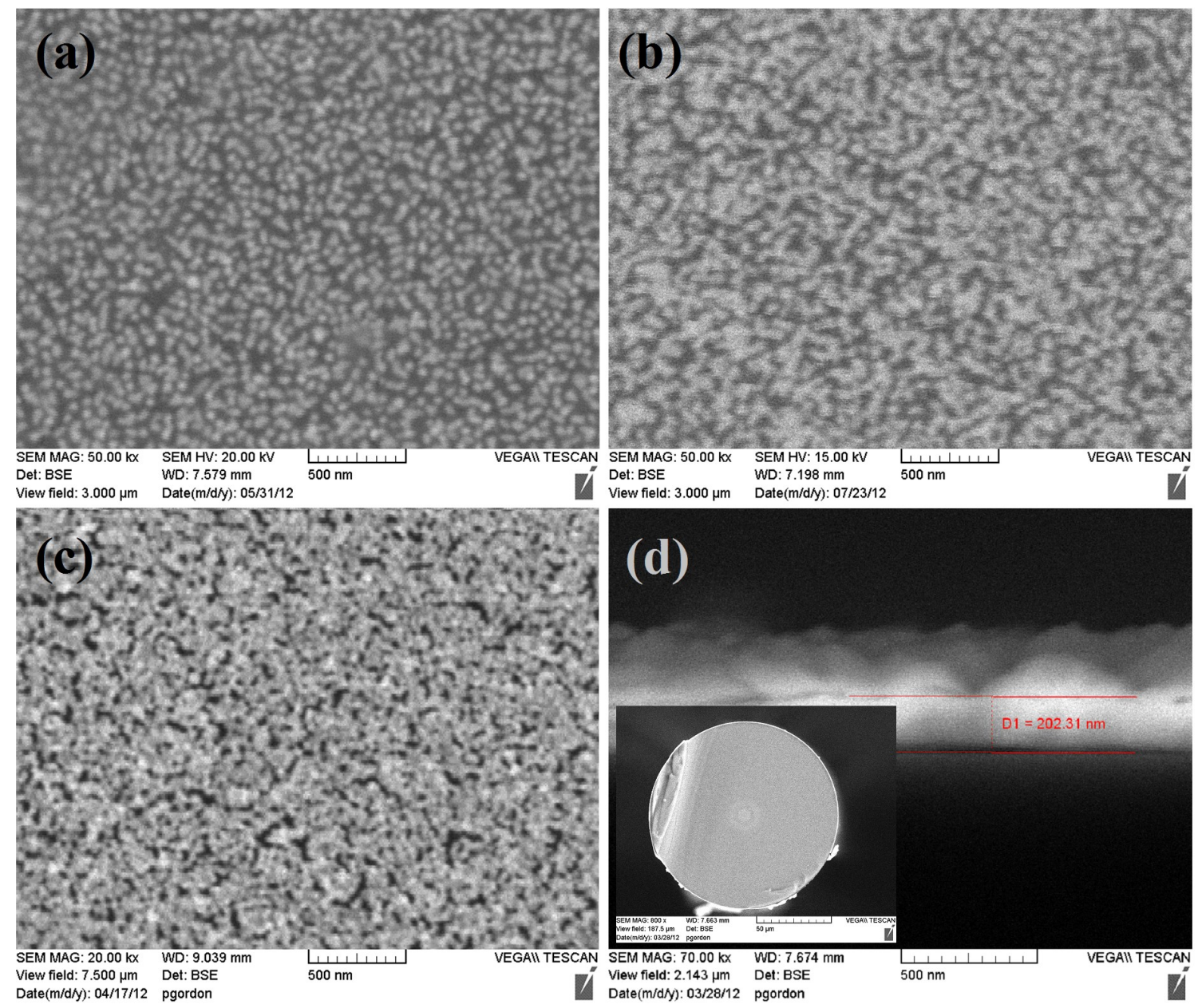

Fig. 3.7. SEM images of gold nanoparticles deposited on the TFBG surface with different deposition times (with a scale of $500 \mathrm{~nm}$ ). (a) Beginning, (b) middle, and (c) end of TFBG spectral evolution. (d) Sectional image of gold film in a small area with an inset of coated TFBG sectional view.

In order to further investigate the origin of the results obtained, we obtained scanning electron microscopy (SEM) images of the surface morphology of the gold nanoparticles deposited on three TFBG samples with different amounts of precursor in the vial (yielding different thicknesses and shorter process durations). Figure 3.7 shows the surface morphology of the growing gold film at beginning (a), middle (b), and end (c) of the whole TFBG spectral evolution, as determined by comparing the final TFBG spectra with those of the "full" process shown in Figs. 3.5 and 3.6. Also included (Fig. 3.7(d)) is an image of one of the fiber cross 
sections that were obtained to estimate the average coating thickness for each case. This correlation yields average thicknesses of 50,100, and $200 \mathrm{~nm}$ for films equivalent to process durations of 20,35, and 80 seconds on Fig. 3.7 (i.e. before the point of maximum attenuation, near the maximum and, finally later in the deposition). Average grain sizes were measured from SEM images to grow linearly with time from $55 \pm 3 \mathrm{~nm}$ at 21 seconds, to $122 \pm 10 \mathrm{~nm}$ at 50 seconds. Individual grains become difficult to identify beyond that time. The films are all quite rough but the metal coverage increases from very sparse (46.41\%), to relatively dense (72.92\%) (but with gaps remaining), and finally to complete, although with a rough top surface. Finally, two further TFBGs were prepared with thicknesses corresponding to deposition times of $20 \mathrm{~s}$ and 40 s, i.e. when the coated TFBG have the largest amplitude differences between S- and Presonances according to Fig. 3.6.

The last two gratings were used to investigate how much of the cladding guided light was scattered by the coatings under these circumstances. We used a broad band source (BBS), polarization controller, and optical spectrum analyzer (OSA) to achieve spectra with precise Sand P-polarized input light, and then imaged the IR-scattering emitted at right angle from the fiber axis with an infrared camera. The measured spectra and associated IR-scattering images with S- and P-polarized light are shown in Fig. 3.8. By comparing the spectra and the images of the two TFBGs, it is very clear that there is a strong correlation between the scattered light intensity and attenuation of the TFBG resonances (the brightest scattering occurring for Fig. 3.8(c), P-polarization, that also shows the strongest resonance amplitude attenuation). In comparison, uncoated TFBGs with similar grating periods look totally dark at these wavelengths because the evanescent field of cladding modes is non-radiative in the direction away from the fiber surface. 

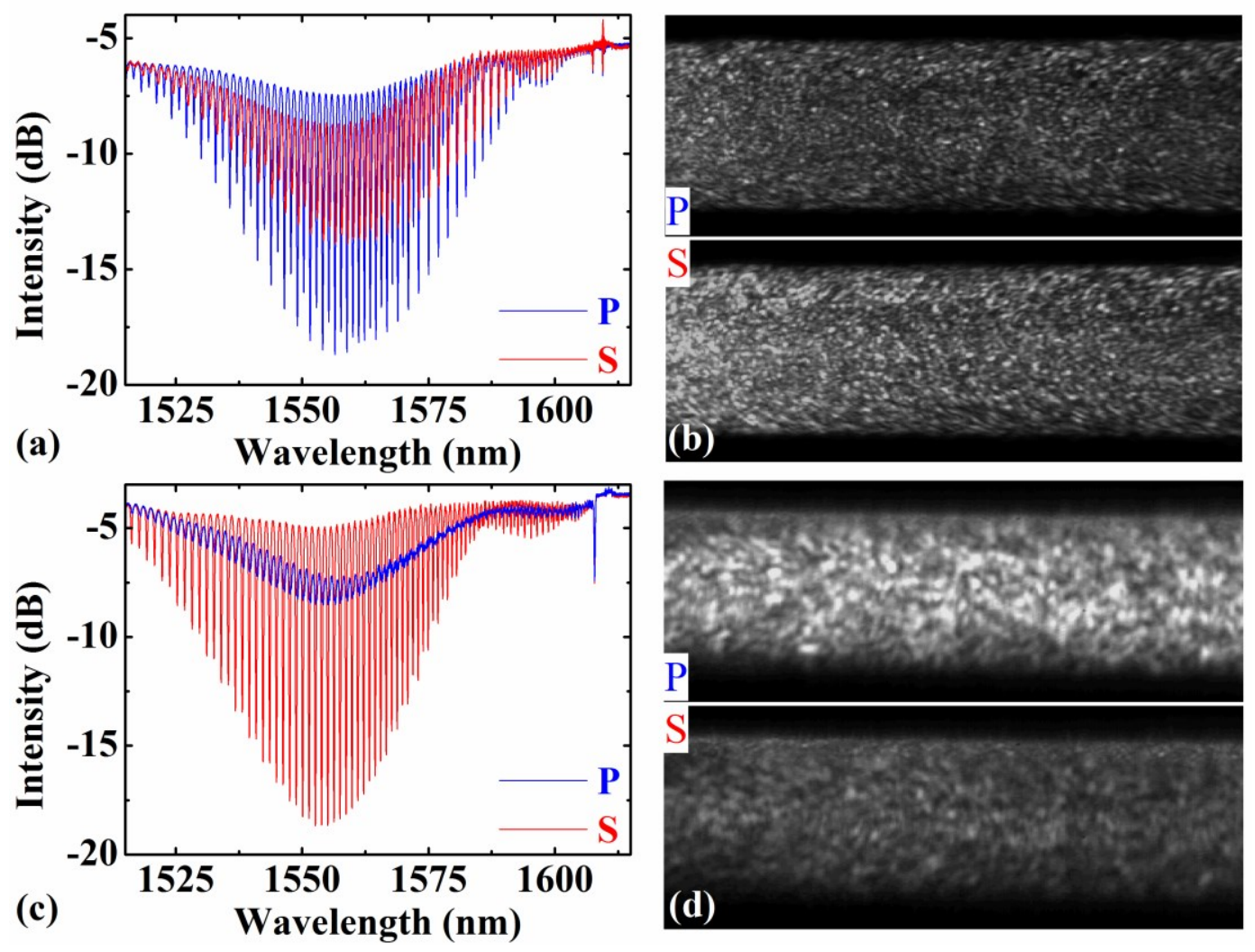

Fig. 3.8. Reflective transmission spectra and IR-scattering images under S- and P-polarized lights of the coated TFBG with spectral response times of $\sim 20 \mathrm{~s}(\mathrm{a}, \mathrm{b})$ and $40 \mathrm{~s}(\mathrm{c}, \mathrm{d})$.

\subsection{Discussions}

It is now clear that the attenuation of the amplitudes is mostly due to scattering by the metal nanoparticles rather than absorption of light by the metal. Furthermore we can extrapolate this finding by claiming that the full recovery of the resonance amplitudes (both S- and P-polarized) arises when the coatings "fill up" and no longer allow the light to leak out (this spectral recovery was also observed for thick electro-plated gratings in [9]. The almost full recovery of the resonance amplitudes also confirm that very little energy is dissipated in the metal itself, otherwise the resonances would remain lossy, losing amplitude and increasing in spectral width as a result. 
The peculiar wavelength shift differences observed between the two polarization states can be explained by the granular nature of the films grown. Based on previous reports about the anomalous permittivity of thin metallic film $[10,17,18]$, the real part of the complex refractive index of a thin film composed of gold nanoparticles increases significantly above the value for bulk gold (a value near 0.55 at these wavelengths) when the particle densities are sparse and the sizes are small. So during the initial growth of the nanoparticles, a thin material layer with a relatively large average refractive index is formed on the cladding, resulting in a red-shift of both kinds of cladding mode resonances. Recalling from elementary electromagnetic theory that tangential fields cannot penetrate high-conductivity metals, S-polarized light (tangential to the cladding surface) can only exit the cladding through the gaps in the coating, while P-polarized light (normal to the interface) can tunnel across the gold particles and also exits from the air gaps. In order to confirm this idea and get a deeper insight into the experimentally observed process, the solution of the full problem of the interaction of the evanescent field of various cladding modes with the films shown in Fig. 3.7 will require exhaustive additional investigations. In the meantime, in order to validate some of the hypotheses brought forth to explain our experimental results, 2D-finite difference time domain (FDTD) numerical simulations of a much simplified configuration were carried out (with software from Lumerical Solution, Inc.). We modeled a linear array of conducting disks with a diameter of $40 \mathrm{~nm}$ and a center-to-center spacing of 50 nm (Fig. 3.9). The disks were surrounded with air and had a complex refractive index ( $n$-ik) of 0.51-i10.8. The simulation domain was meshed with cells on a $0.2 \mathrm{~nm}$ grid. The incident electromagnetic field was a plane wave pulse centered at wavelength of $1.5 \mu \mathrm{m}$ with its electric field vector $\mathbf{E}$ lying in the plane of the simulation domain. Two orthogonal cases were investigated: a) wave incident from the bottom of the image with the vector $\mathbf{E}$ oriented 
horizontally (corresponding to azimuthally polarized modes, from S-polarization coupling); b) wave incident from the left with the vector E oriented vertically (approximating a radially polarized evanescent field, i.e. a mode generated by P-polarized light).
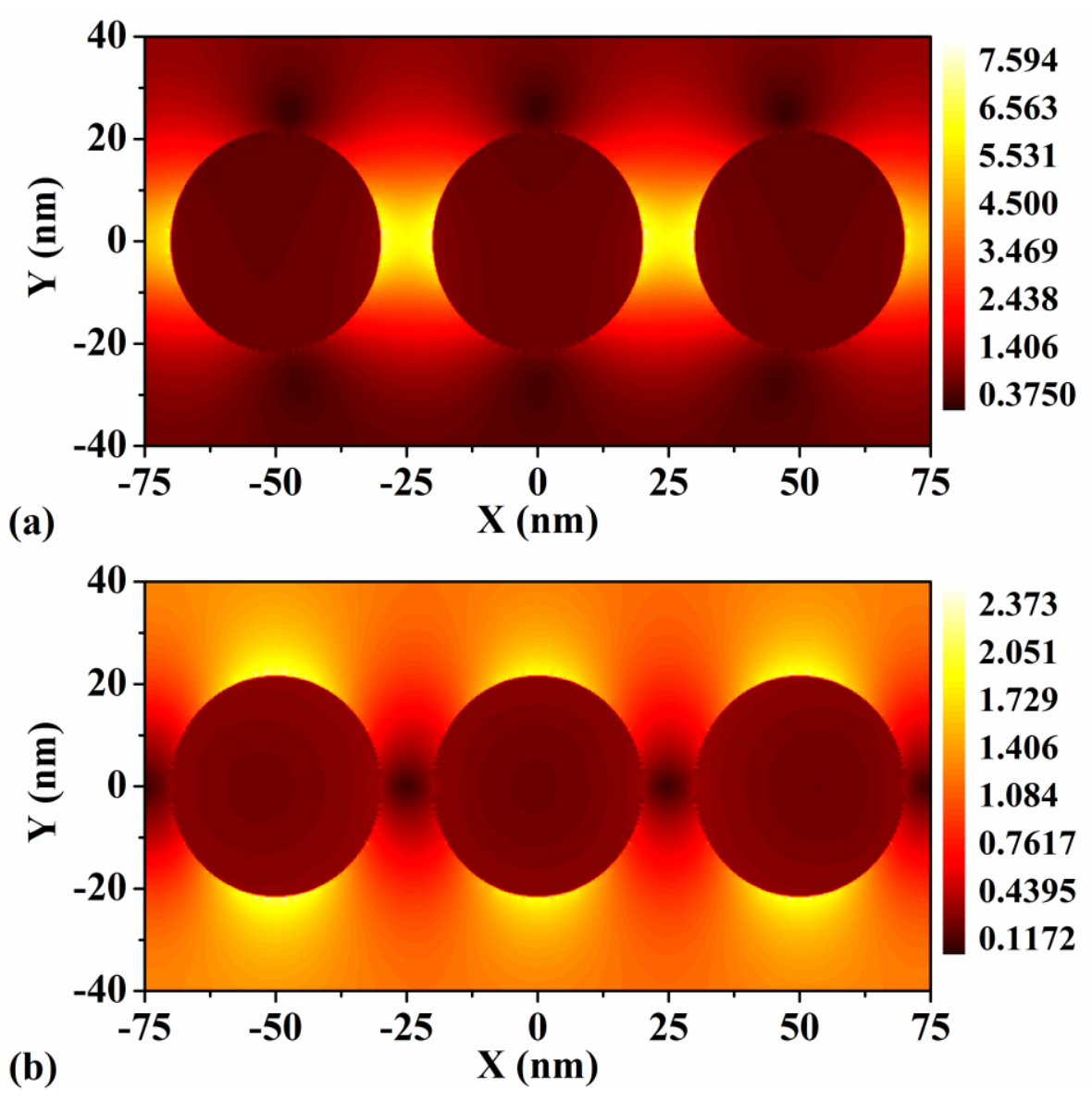

Fig. 3.9. Simulated intensity distributions of electric fields on single layer gold nanoparticles interacted by S- (a) and P-polarized (b) light in free space. The incident light propagates along Y-axis in S case, while along $\mathrm{X}$-axis in $\mathrm{P}$ case.

The optical intensity distributions around the metal disks under these two excitation conditions are shown in Fig. 3.9. It is very clear that the light with the electric field tangential to the nanoparticle layer (S-polarization) is strongly localized between the metal particles but is forbidden to go through the particles themselves. On the other hand, light associated with Ppolarized excitation of the TFBG (case b) has strong electric field maxima on the top and bottom 
of the nanoparticles while being "forbidden" in between because it is polarized tangentially to the metal there (and the gap is too narrow). Given these simulated results, we can infer that as the particles grow and the air gaps decrease, light from S-polarized resonances reaches a point, when most of the gaps between particles close, where it can no longer penetrate the nanoparticle coating and becomes bounded by what appears from the inside of the fiber as a continuous (i.e. bulk) metal film with a low refractive index of 0.5 , hence the large sudden blue shift of the resonances. On the other hand, when the air gaps close the P-resonances continue to probe deeply into the metal and to continue to red shift slowly as long as their evanescent field can tunnel across the whole layer thickness (apart from some irregular behaviour in the transition zone where the gaps become small before closing). The net result is that the presence of the nanoparticles introduces loss for the cladding modes propagating underneath. This loss is reflected in the attenuation of the cladding mode resonances (they become less pronounced, as seen on Figs. 3.5 and 3.6, and especially 3.8(c)). Figure 3.8 clearly shows that most of the attenuation arises because of scattering, by opposition to absorption by the metal. If absorption was involved, the resonances would not recover their full amplitudes (see Fig. 3.6(a)) as the films become continuous and thicker. Finally, for the most strongly attenuated cladding modes (and strongest scattered light), the total net loss of the core guided mode for P-polarized light (as determined by its transmission spectrum) is only about $5 \mathrm{~dB}$ : this means that about $70 \%$ of the light is extracted from the core and transferred to the nanoparticles and their surroundings.

Regardless of the validity of these hypotheses, the main conclusion remains that we have identified, from the TFBG spectral signature, the conditions where the HE (and TE) cladding modes become isolated from the fiber surroundings while allowing EH (and TM) modes to tunnel across and scatter efficiently off the nanoparticles. 


\subsection{Conclusion}

We have demonstrated that there is a significant correlation between the near infrared polarized transmission spectra of $10^{\circ}$ TFBG inscribed in standard single mode fibers and the optical properties gold particle coatings with thicknesses ranging from zero to $\sim 200 \mathrm{~nm}$, and formed using a recently developed chemical vapour deposition process. In particular we identified spectral signatures corresponding to coatings yielding different states of light confinement, scattering, and polarization. For instance, for films with particle sizes near $50 \mathrm{~nm}$ and $100 \mathrm{~nm}$ respectively we can selectively scatter out of the fiber light polarized predominantly azimuthally or radially and thus control the plasmonic enhancement properties due to these disordered nanostructured metal films $[19,20]$. However, the optimum film thickness and uniformity will vary from application to application and the scattering properties further depend on the size of individual nanoparticles and the porosity of the films, parameters that cannot be controlled independently at the present time with this method. What we show here is that the film thickness can be controlled by stopping the deposition before the precursor is exhausted and that several film conditions can be obtained as a result. Further experiments with different process temperatures, residual pressure in the chamber, or additional reactants would provide a richer parameter space but the results obtained so far are sufficient for developing interesting devices. It is to be pointed out that the purpose here was not to control the polarization of the guided light, as can be done very efficiently using un-coated TFBGs with large tilt angles [21], or by using graphene coatings on side polished fibers for instance [22], but rather to excite coatings on fibers with radially or azimuthally polarized light at infrared wavelengths. As a side benefit, we have also confirmed the usefulness of the TFBG as a process monitor for thin film coatings of CVD gold on other substrates in the same process chamber, in situ and in real time. 


\subsection{References}

1. J. J. Mock, R. T. Hill, Y.-J. Tsai, A. Chilkoti and D. R. Smith, "Probing dynamically tunable localized surface plasmon resonances of film-coupled nanoparticles by evanescent wave excitation," Nano Lett. 12, 1757-1764 (2012).

2. L. Tong, V. D. Miljkovic and M. Kall, "Alignment, rotation, and spinning of single plasmon nanoparticles and nanowires using polarization dependent optical forces," Nano Lett. 10, 268-273 (2010).

3. Y.-C. Lu, R. Geng, C. Wang, F. Zhang, C. Liu, T. Ning and S. Jian, "Polarization effects in tilted fiber Bragg grating refractometers,” J. Lightwave Technol. 28, 1677-1684 (2010).

4. J. Albert, L.-Y. Shao and C. Caucheteur, "Tilted fiber Bragg grating sensors," Laser Photonics Rev. 7(1), 83-108 (2013).

5. Y. Shevchenko, C. Chen, M. A. Dakka and J. Albert, "Polarization-selective grating excitation of plasmons in cylindrical optical fiber," Opt. Lett. 35, 637-639 (2010).

6. C. Caucheteur, Y. Shevchenko, L.-Y. Shao, M. Wuilpart and J. Albert, "High resolution interrogation of tilted fiber grating SPR sensors from polarization properties measurement," Opt. Express 19, 16561664 (2011).

7. J. Pollet, F. Delport, K. P. F. Janssen, K. Jans, G. Maes, H. Pfeiffer, M. Wevers and J. Lammertyn, "Fiber optic SPR biosensing of DNA hybridization and DNA-protein interactions," Biosens. Bioelectron. 25, 864-869 (2009).

8. T. Schuster, R. Herschel, N. Neumann and C. G. Schaeffer, "Miniaturized long-period fiber grating assisted surface plasmon resonance sensor," J. Lightwave Technol. 30, 1003-1008 (2012).

9. A. Bialiayeu, C. Caucheteur, N. Ahamad, A. Ianoul and J. Albert, "Self-optimized metal coating for fiber plasmonics by electroless deposition,” Opt. Express 19, 18742-18753 (2011).

10. L.-Y. Shao, J. P. Coyle, S. T. Barry and J. Albert, "Anomalous permittivity and plasmon resonances of copper nanoparticle conformal coating on optical fibres," Opt. Mater. Express 1, 128-137 (2011).

11. T. J. J. Whitehorne, J. P. Coyle, A. Mahmood, W. H. Monillas, G. P. A. Yap and S. T. Barry, "Group 11 amidinates and guanidinates: potential precursors for vapour deposition,” Eur. J. Inorg. Chem. 21, 3240-3247 (2011).

12. C.-F. Chan, C. Chen, A. Jafari. A. Laronche, D. J. Thomson and J. Albert, "Optical fiber refractometer using narrowband cladding-mode resonance shifts," Appl. Opt. 46, 1142-1149 (2007).

13. I. D. Villar, I. R. Matias, F. J. Arregui and M. Achaerandio, "Nanodeposition of materials with complex refractive index in long-period fiber gratings," J. Lightwave Technol. 23, 4192-4199 (2005). 
14. C. Caucheteur, C. Chen, V. Voisin, P. Berini and J. Albert, "A thin metal sheath lifts the EH to HE degeneracy in the cladding mode refractometric sensitivity of optical fiber sensors," Appl. Phys. Lett. 99, 041118 (2011).

15. The cylindrical Finite Difference Fiber Mode Solver included in FIMMWAVE by Photon Design, http://www.photond.com/products/fimmwave/fimmwave_features_25.htm

16. I. Horcas, R. Fernandez, J. M. Gomez-Rodriguez, J. Colchero, J. Gomez-Herrero and A. M. Baro, "WSXM: A software for scanning probe microscopy and a tool for nanotechnology," Rev. Sci. Instrum. 78, 013705 (2007).

17. M. Chen and R. G. Horn, "Refractive index of sparse layers of adsorbed gold nanoparticles," J. Colloid Interf. Sci. 315, 814-817 (2007).

18. W.-J. Lee, J.-E. Kim, H. Y. Park, S. Park, M.-S. Kim, J. T. Kim and J. J. Ju, "Optical constants of evaporated gold films measured by surface plasmon resonance at telecommunication wavelengths," J. Appl. Phys. 103, 073713 (2008).

19. E. Castanié, V. Krachmalnicoff, A. Cazé, R. Pierrat, Y. De Wilde and R. Carminati, "Distance dependence of the local density of states in the near field of a disordered plasmonic film," Opt. Lett. 37, 3006-3008 (2012).

20. S. H. Choi, B. Kwak, B. Han and Y. L. Kim, "Competition between excitation and emission enhancement of quantum dots on disordered plasmonic nanostructures," Opt. Express 20, 1678516793 (2012).

21. T. A. Strasser, P. S. Westbrook and T. Erdogan, "In-line polarimeter using blazed fiber gratings," Photon. Technol. Lett. 12, 1352-1354 (2000).

22. Q. Bao, H. Zhang, B. Wang, Z. Ni, C. H. Y. X. Lim, Y. Wang, D. Y. Tang and K. P. Loh, "Broadband graphene polarizer," Nat. Photon. 5, 411-415 (2011). 


\section{Chapter 4: Effective Permittivity of Ultrathin Chemical}

\section{Vapor Deposited Gold Films on Optical Fibers at Infrared}

\section{Wavelengths ${ }^{2}$}

The geometry and size-dependent effective medium properties of ultrathin gold films deposited on the bare cladding of single mode optical fibers by chemical vapour deposition (CVD) are characterized by measuring the polarized transmission spectra of in-fiber gratings at wavelengths near $1550 \mathrm{~nm}$. The real part of the complex refractive indices of films with average thicknesses ranging from 6 to $65 \mathrm{~nm}$ are about 10 times higher than that of bulk
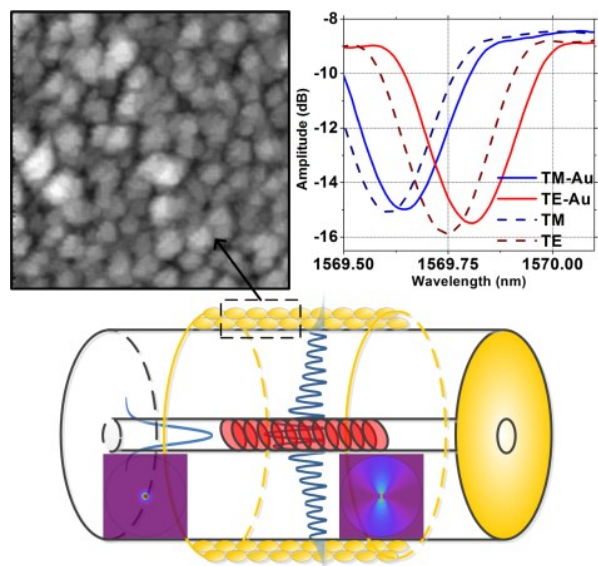
gold at these wavelengths, while the imaginary part values are 2 orders of magnitude lower. The films are essentially isotropic, apart from a small increasing dichroism between the in-plane and out-of-plane component of the imaginary part of the refractive index at thicknesses larger than $25 \mathrm{~nm}$. Unlike gold films prepared by other means, the optical properties of the coatings do not converge rapidly towards bulk values at thicknesses larger than $10 \mathrm{~nm}$ but remain characteristic of gold films prepared by very slow physical deposition processes. The modified Clausius-Mossotti theory for anisotropic structures was used to confirm that the observed properties arise from a persistent granularity of the film at larger thicknesses, with metal filling fractions increasing from $30 \%$ to $68 \%$ and particle aspect ratios from 0.8 to 1.0 (spherical). These conclusions are supported by nanoparticle shape measurements obtained by Atomic Force Microscopy and Scanning Electron Microscope images.

\footnotetext{
${ }^{2}$ Reproduced with Permission from "W. Zhou, D. J. Mandia, M. B.E. Griffiths, S. T. Barry, and J. Albert, 'Effective permittivity of ultrathin chemical vapor deposited gold films on optical fibers at infrared wavelengths,' The Journal of Physical Chemistry C 118(1), 670-678 (Jan. 2014)." Copyright 2014, American Chemical Society.

Author contributions: J.A. conceived the basic idea of a TFBG model for analyzing the optical properties of metal coatings. J.A. planned, supervised and supported the experimental and simulation implementation. W.Z. fabricated the TFBG probes, built the experimental setup, processed the experimental data, and performed the numerical simulations. M.B.E.G. made the gold precursors. D.J.M. operated the gold CVDs, and took the SEM and AFM images. W.Z., D.J.M., and J.A. wrote the manuscript. The project was organized and coordinated by J.A. and S.T.B.
} 


\subsection{Introduction}

The electromagnetic field intensity in the immediate vicinity of various ultrathin metal films and nanostructures can be enhanced by several orders of magnitude [1-5]. This phenomenon has led to a number of applications in plasmonic sensing [6,7], surface-enhanced Raman scattering [8], and thermo-plasmonics [9]. There are many of those applications that can be implemented using coated optical fibers and it is important in such cases that the thin metals films be coated uniformly around the fiber circumference. This is most easily done using liquid phase or gas phase chemical deposition techniques, because the more widely used thin film coating techniques such as evaporation and sputtering cannot reliably produce uniform thicknesses around curved surfaces. Once fabricated, the intensity and wavelength dependence of the plasmonic enhancement are related to the material and geometric parameters of metallic nanostructures: the size $[2,3]$, pattern $[4,5]$, as well as the permittivity of the metal and surrounding material $[1,10]$. While size and geometric patterns can be extracted with modern imaging and surface profilometric tools, the complex permittivity is typically obtained from the reflection and transmission measurements of the metallic film with analysis using Drude model parametrization and Kramers-Krönig relations [11-13]. However, it is now clear that these approaches, which were developed for bulk (i.e. continuous) metals films are less suitable for ultrathin metals [14] or aggregates of metal nanoparticles (NPs) with individual sizes of the order $50 \mathrm{~nm}$ and less [15]. In the latter case, it was suggested to modify the collision frequency of free electrons by an approximate, empirically determined size-dependent parameter in the Drude model [16]. However, it remains difficult to measure the complex permittivity of such nanostructures directly without resorting to various theoretical models for the electromagnetic mixing formulas between the NPs and their surroundings [17]. It is therefore desirable to 
investigate the permittivity or complex refractive index of ultrathin metal film by several different optical methods. Spectroscopic ellipsometry can obtain the complex permittivity of homogeneous thin films, with decreasing accuracy and resolution as the films get thinner $[18,19]$. Furthermore, when the films are not isotropic this method becomes significantly less reliable and requires non trivial computations to extract the five parameters of interest (real and imaginary part of the refractive index for TE and TM light, plus the thickness if it is not determined by other means). For metallic thin films, i.e. for films with a negative real part of the permittivity, the surface plasmon resonance (SPR) technique can also be used to investigate the complex refractive index and thickness of metal films [20,21], but only for TM polarized light and only for a limited range of dielectric refractive indices for the substrate and cover materials of the thin films. In particular, SPR cannot be excited at an ultrathin metallic film-air interface at infrared wavelengths because the anomalous size-dependent permittivity of the metal becomes positive [22]. In a more recent advance, interferometric picometrology was developed to measure the inplane component of the complex refractive index of ultrathin gold films with a single wavelength, linearly polarized light beam at normal incidence [23]. In this new method, the complex index can be calculated from the amplitude change and phase shift of the complex reflection coefficient.

A common issue with all thin film measurement techniques is that they are not well suited for curved surfaces. For the geometry considered here, a conformal coating on the surface of a $125 \mu \mathrm{m}$ diameter optical fiber, the curvature represents a challenging measurement problem. Finally, for very thin films that perturb electromagnetic waves very little, the best way to increase the measurement accuracy is to use frequency-domain techniques, such as measuring the frequency shifts of resonator structures that have high quality (Q) factors [24-26]. It is this type of approach that is presented here with a resonating waveguide structure that is especially 
easy to fabricate and to measure [27]. The objective of the present study is two-fold: the development of a technique to measure the permittivity of thin metal films on optical fibers, and the characterization of such coatings made by a relatively high temperature chemical vapor deposition (CVD) process.

The polarized evanescent electric field of resonantly excited optical fiber modes is used to probe ultrathin gold films deposited on the surface of the fiber in order to investigate the effective medium properties (refractive index and absorption) of the films at infrared wavelengths. Conformal gold films with different thicknesses were deposited on the cladding of optical fibers by CVD, as previously reported for other metals or gas precursors [28-30]. The excitation of the probing optical modes was carried out with a tilted fiber Bragg grating (TFBG) inscribed in the core of the optical fiber over lengths of several millimeters. The grating couples up to $90 \%$ of the light launched in the core to individual cladding guided modes and thus provides high signal to noise ratio resonances with Q-factors (resonance wavelength/resonance width) of $\sim 10,000$ [27]. Changes in the spectral properties of these cladding mode resonances were used to measure the complex permittivity of the coatings averaged over the full length of the gratings ( $5 \mathrm{~mm}$ in these experiments). The method further allows the separate measurement of film properties for TE (in-plane electric field) and TM light (out of plane) and as a result can detect the presence of birefringence or dichroism. These polarization-resolved measurements were obtained by exciting separately cladding-guided modes with azimuthally or radially polarized electric field at the cladding-coating boundary, which correspond respectively to TE and TM light polarizations for the electric field penetrating into the deposited coatings [31]. In parallel, scanning electron microscope (SEM) and atomic force microscope (AFM) images of the films that were deposited were used to provide shape, roughness, and average thickness data, 
thus completing the characterization of the films. An effective medium model for the deposited thin films was used to determine their complex permittivity in terms of the measured parameters. Finally, we extracted the metal volume filling fraction and aspect ratio of the NPs in the films by modelling the measured effective medium permittivity of the films composed of ellipsoidal gold NPs under TE and TM polarizations with a modified Clausius-Mossotti equation.

\subsection{Experimental section}

TFBG probe fabrication. TFBGs were written in hydrogen-loaded CORNING SMF-28 fiber with a pulsed $\mathrm{KrF}$ excimer laser using the phase-mask method [27]. This fiber is a standard telecommunication grade fiber that guides only one core mode at wavelengths between 1300 and $1620 \mathrm{~nm}$. The grating period $(555.2 \mathrm{~nm})$ is chosen to reflect the core guided light at the "Bragg" wavelength around $1610 \mathrm{~nm}$. At shorter wavelengths between 1520 and 1600 the core guided light is coupled to the $125 \mu \mathrm{m}$ diameter cladding of the fiber, which acts as a highly multitude waveguide. Figure 4.1 shows the reflected transmission spectrum of a TFBG in air prior to coating. Each of the resonances corresponds to a wavelength at which core guided light is coupled to a specific group of polarized cladding modes. The length of the TFBG was chosen to be only $5 \mathrm{~mm}$ long in order to minimize the impact of an eventual thickness gradient on the measured quantities if the deposition was not uniform. A tilt angle of $10^{\circ}$ was chosen in order to excite a large number of strong, high-order cladding mode resonances across the measurement range of our instrumentation (centered around $1550 \mathrm{~nm}$ ). While TFBGs are normally measured in transmission, we implemented a reflective sensing configuration in order to have a single entry port in the deposition chamber and to avoid bending the fiber (Figure 4.2). This configuration was implemented by cleaving the fiber $1.5 \mathrm{~cm}$ downstream from the TFBG and coating the flat end with a sputtered gold mirror. While the outside face of the end mirror also 
gets coated during CVD, this has no influence on the measured properties as the mirror is thick enough to be impervious to additional coatings. A consequence of the reflective configuration is that the core guided light goes through the grating twice and therefore that the grating power transfer function is squared.

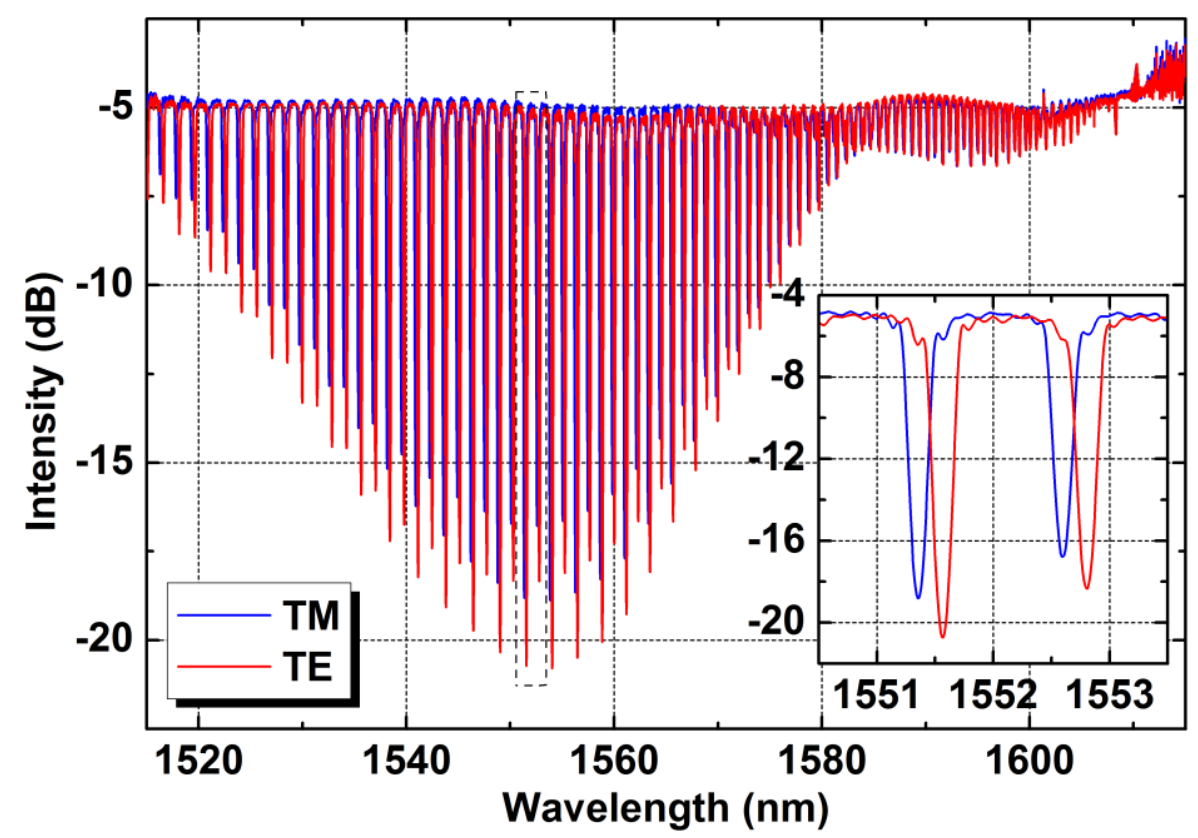

Fig. 4.1. Typically reflected transmission spectrum of bare TFBG under TM and TE polarizations.

Sample preparation. Figure 4.2(a) illustrates the experimental setup of the gold CVD system. The fiber was located in a stainless steel deposition chamber, which itself was mounted within a furnace. An evaporator containing a vial loaded (under $\mathrm{N}_{2}$ ) with a gold(I) iminopyrrolidinate $\left(\left[\mathrm{Au}\left(\mathrm{Me}_{2}-{ }^{\mathrm{t}} \mathrm{Bu}-\mathrm{ip}\right)\right]_{2}\right) \mathrm{CVD}$ precursor was situated at the end of the chamber and surrounded by heating tape for external resistive heating by a Variac. At the beginning of the experiment the chamber was evacuated to a base pressure of $5 \mathrm{mTorr}$ and then the furnace temperature (substrate temperature) was set to $350{ }^{\circ} \mathrm{C}$. After the substrate achieved constant temperature, the evaporator was heated to a temperature of $\sim 160{ }^{\circ} \mathrm{C}$ to initiate volatilization of the precursor. Onset of deposition occurs when the evaporator temperature reaches $\sim 130{ }^{\circ} \mathrm{C}$, at which point the 
vaporous precursor saturates the chamber, effectively depositing on all exposed surfaces including the cladding of the TFBG. The deposited precursor then thermally decomposes into pure gold by a reaction that is still under investigation but that was confirmed to occur at temperatures above $300{ }^{\circ} \mathrm{C}$ [32]. High-resolution X-ray photoelectron spectroscopy (XPS) analysis performed on witness slides coated with this gold material showed no significant or detectable impurities. Since the gold precursor is thermally stable between $150{ }^{\circ} \mathrm{C}$ and $300{ }^{\circ} \mathrm{C}$, the thickness of the gold film can be controlled by adjusting the amount of precursor, the temperature of the deposition chamber, and the location of the samples to be coated relative to the inlet of the evaporator into the deposition chamber. A small piece of bare optical fiber and witness slides were coated simultaneously for ex situ microscopic imaging of the surface morphology of the gold films. These additional samples were located 3-5 $\mathrm{mm}$ away from the TFBG to ensure a similar coating environment.

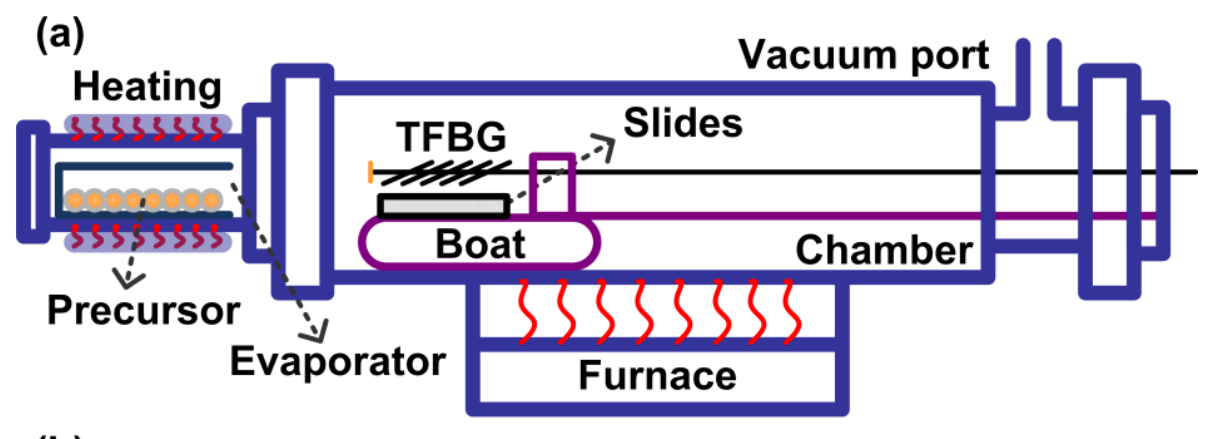

(b)

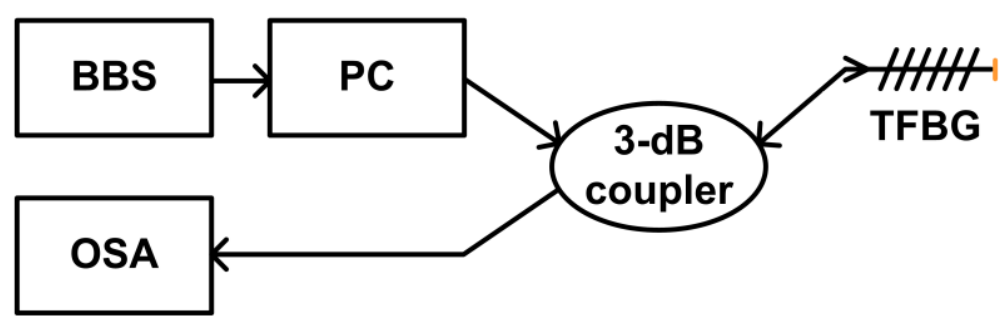

Fig. 4.2. Schematic diagram of experimental process: (a) Gold CVD setup for optical fiber; (b) TFBG spectral measurement system. A source (BBS) followed by a polarization controller generates a broad spectrum of light that is linearly polarized either parallel (TM) or perpendicular (TE) to the tilt plane of 
the grating. The core-guided light going through the grating is reflected by a gold mirror, goes through the grating a second time and is detected by an optical spectrum analyzer (OSA).

Optical measurements. The measurement system is shown in Figure 4.2(b). The fiber-coupled broadband source (JDSU BBS1560) launches light from 1520 to $1620 \mathrm{~nm}$ in the same type of single mode telecommunication fiber is used for the TFBG. Even though the fibers are not of the polarization maintaining type, a polarization controller (JDSU PR2000) can be used to generate a state of polarization that will evolve to a linear state either parallel (p-polarized, or TM) or perpendicular (s-polarized or TE) at the grating. As long as the fiber paths are not moved during the experiments, polarization remains stable. As indicated earlier, when the polarization state of incident light at the TFBG contains only TE- or TM-polarized light, the electrical field of the excited high-order cladding modes are predominantly azimuthal or radial at the cladding boundary respectively and the associated TFBG resonances occur at different wavelengths [27,31]. The light transmitted through the grating twice returns towards the light source in the same fiber and a 50:50 fiber coupler picks off part of the reflected light to be measured by an ANDO AQ6317B optical spectrum analyzer with a nominal resolution of $0.01 \mathrm{~nm}$.

By using the TFBG spectral measurement system shown in Figure 4.2(b), the polarization-dependent permittivity of the thin gold film can be interrogated by in-plane (TE) and out-of-plane (TM) light and measured very easily by the polarization-dependent spectra of the TFBG. The system is completely fiber-coupled and therefore no optical alignment is required. While TFBGs can usually be used to monitor coatings formation (and in situ process temperature) during deposition [28,29], it was not possible here. The thermal decomposition temperatures exceed the range over which FBGs are stable and therefore induce irreversible spectral changes upon return to room temperature. Therefore, all results shown here compare a coated and bare TFBG, with the bare spectrum measured after removing the coating (instead of 
prior to deposition), in order to remove the effect of the thermally induced spectral changes. Separate TFBGs were used for each coating thickness, and different thicknesses were obtained by changing the amount of gold precursor in the evaporator. Coatings were removed by etching in Aqua Regia (a fresh mix of nitric acid and hydrochloric acid with volume ratio of 1:3). This methodology yields accurate comparative spectral information about the wavelength shift and amplitude attenuation of the TFBG resonances induced by the gold coatings.

Microscopy. Scanning electron microscopy (SEM) and atomic force microscopy (AFM) were performed on Tescan and Agilent (Molecular Imaging) PicoSPM II systems, respectively. Imaging of fiber samples and coated witness slides was carried out by SEM for grain size analysis and AFM for surface morphology and thickness. In view of the roughness of the deposited coatings, the thickness data was calculated from the average step height at the boundary of films interrupted by abrasion of a small section.

\subsection{Results}

Surface characterization of gold CVD films. Figure 4.3 shows AFM images (a-d) and effective height profiles (e) of the gold films with various effective thicknesses. The effective height profiles were obtained by adding the average step height from scratched samples to the middle of the AFM surface height distributions of undisturbed coatings. As evidenced on the AFM images, the gold films are all composed of roughly spherical NPs regardless of average thickness. The thinner film $(6 \mathrm{~nm})$ appears to be made up of individual NPs (because the height distribution extends over $\pm 6 \mathrm{~nm}$ around the mean), while the AFM profiles of thicker films do not penetrate down to the substrates. In fact, the size distribution is nearly identical $( \pm 15 \mathrm{~nm})$ for the 40 and the $65 \mathrm{~nm}$ thick films. Furthermore, the lateral size of the NPs on the AFM images does not differ much (apart from the thinnest film). In order to eliminate possible measurement artefact 
due to the convolution of the AFM tip size with the profiles of the NPs, SEM analysis was made on the flat witness slides for each thickness, as shown in Figure 4.4. It is important to note that the apparently very high curvature of the fiber surface $(1 /(62500 \mathrm{~nm}))$ should have no effect on the deposition rate for the effective thicknesses used here $(6-200 \mathrm{~nm})$. ImageJ software was used to measure the average lateral sizes of representative samples of the gold NPs at each thickness. The average lateral sizes (approximate diameters) are tabulated in Table 1 along with the thickness parameters extracted from the AFM measurements. The major size differences occur for the thinnest and thickest films measured (6 and $200 \mathrm{~nm}$ respectively), with somewhat more modest changes (lateral sizes between 42.6 and $61.5 \mathrm{~nm}$ ) for thicknesses between 13 and $65 \mathrm{~nm}$. It is very clear that thin gold films deposited by this kind of CVD precursor grow by NP nucleation and do not become more uniform, even for the $200 \mathrm{~nm}$ thick gold film shown in Figure 4.4(f).
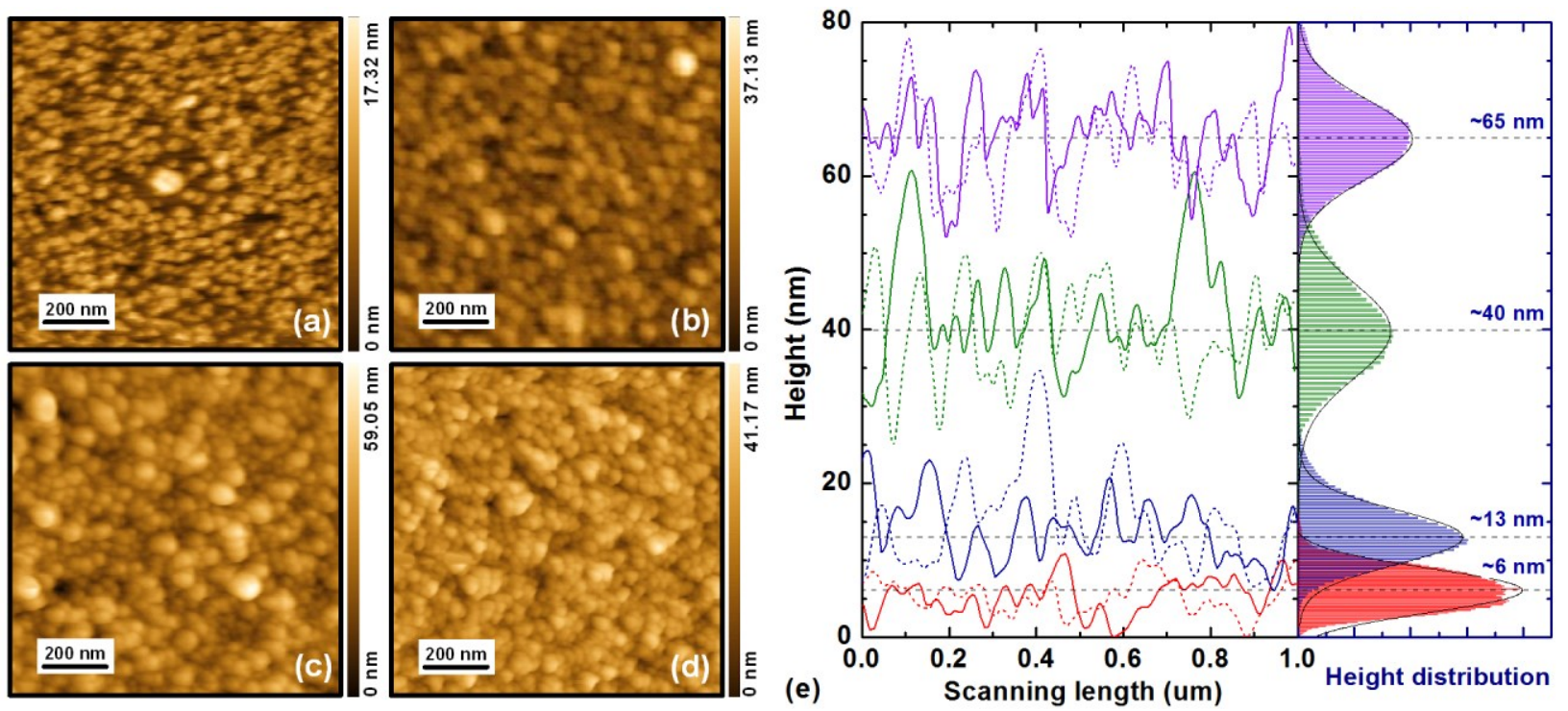

Fig. 4.3. (a-d) AFM images of gold CVD films deposited on planar slides with effective thickness of 6, 13, 40, and $65 \mathrm{~nm}$. (e) Two sample height profiles as well as height histograms of the AFM images shown in (a-d). The centers of the effective height distributions are displaced by the average step height of the film for each sample. 


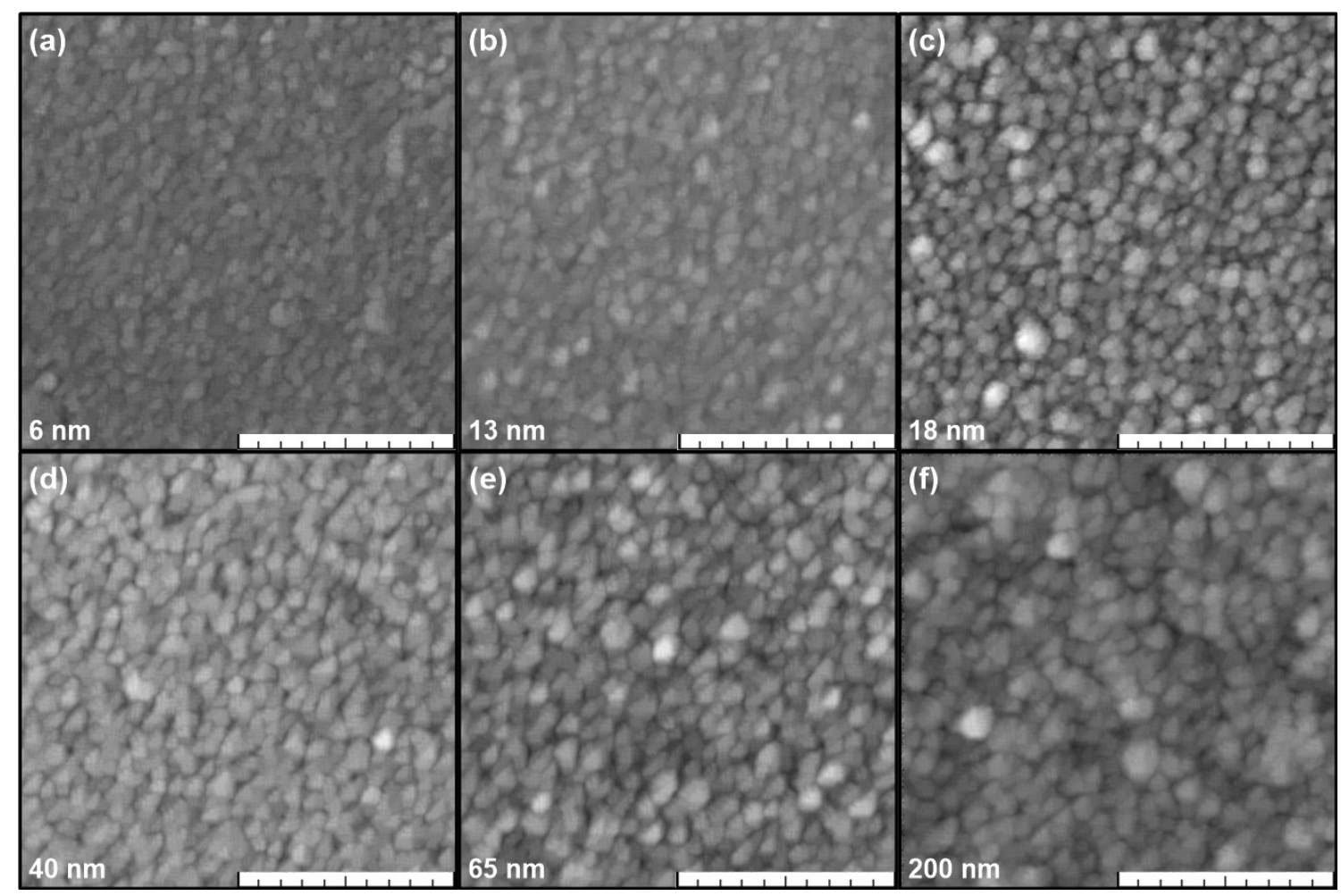

Fig. 4.4. (a-f) SEM images of gold CVD films coated on optical fibers at various effective thicknesses (indicated at bottom-left of each image). The scale bar is $500 \mathrm{~nm}$.

Table 4.1. Average thickness, thickness distribution (standard deviation and full width at half maximum (FWHM) of the Gaussian fitting curve of the height histograms), and average lateral size of gold NPs ( \pm 5 nm) extracted from AFM and SEM pictures of CVD gold samples.

\begin{tabular}{l|llllllll}
\hline Average thickness/nm & 6 & 10 & 13 & 18 & 25 & 40 & 65 & 200 \\
& & & & & & & & \\
\hline Standard deviation/nm & 2.8 & 2.2 & 3.5 & 5.1 & 3.6 & 6.2 & 5.1 & 5.5 \\
& & & & & & & & \\
\hline FWHM/nm & 6.7 & 5.3 & 8.2 & 11.9 & 8.4 & 14.5 & 12.0 & 13.0 \\
& & & & & & & & \\
\hline Lateral size/nm & 36.2 & 40.1 & 42.6 & 54.7 & n.a. & 50.6 & 61.5 & 77.6 \\
& & & & & & & & \\
\hline
\end{tabular}

TFBG spectral responses. Figure 4.5 shows the experimental wavelength shifts and amplitude attenuations of a pair of cladding modes (one TE and one TM) with effective indices near 1.378. 
For each resonance, the peak-to-peak amplitude $\left(A_{p-p}\right)$ is defined as the difference between the minimum transmission of a resonance and the nearest maximum in the spectrum, while the wavelength is determined as the mid-point of the spectral dip. These measured values are used to calculate changes in the effective index of the mode through equations 1 and 2 . The effective index is a measure of the mode phase velocity along the axis of the fiber, and it depends on the wavelength, the material indices, and the waveguide geometry. A mode effective index of 1.378, combined with the refractive index of the fiber cladding (1.4440) corresponds to light from this mode striking the cladding-coating boundary at an angle of incidence of 72 degrees. The addition of a coating on the fiber leads to a change in the real and imaginary parts of the mode effective indices and as a result to a shift of the transmission spectrum resonances (in wavelength and amplitudes). Part (a) of Figure 4.5 shows clear spectral shifts, different for TE and TM modes, even for films thicknesses as small as $6 \mathrm{~nm}$. The TE mode resonance shifts by $1.5 \mathrm{~nm}$ as the film thickness increases to $65 \mathrm{~nm}$, while the TM resonance shifts by only $0.4 \mathrm{~nm}$. This information is not sufficient however because the gold coating also introduces optical loss. In order to measure the imaginary part of the complex permittivity of the film, the increase in mode propagation loss must be measured. This is done by measuring the resonance amplitude, which depends on the grating length, coupling coefficient, and mode loss. The first two parameters do not change when a thin coating is applied (the coupling coefficient could change slightly with loss, but this is a second order effect, negligible for the shifts observed here), so the amplitude shifts can be directly linked to the addition of the lossy coatings. Since different gratings were used for each thickness, the starting values of the TFBG resonance corresponding to the desired cladding mode are different (histograms in the Figure 4.5(b)). As a result, the amplitude changes of the resonances appear irregular (line-symbol curves in Figure 4.5(b)). Fortunately, this is not a 
problem as the algorithm to extract mode loss from the amplitude change is independent of the initial values of the resonance amplitude [33].

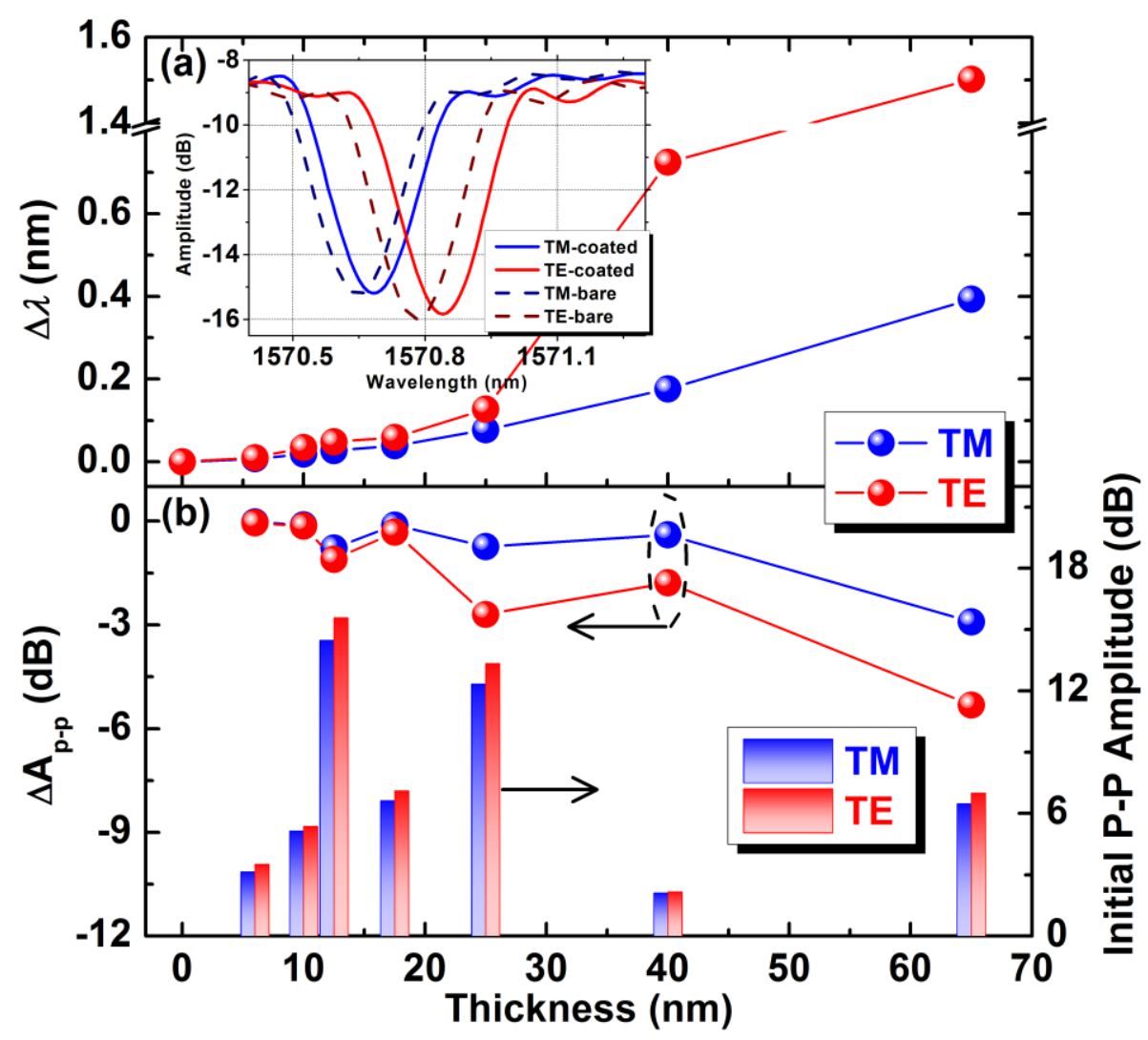

Fig. 4.5. Relative wavelength shift (a) and peak-to-peak amplitude attenuation (b) of TE- and TMpolarized cladding mode resonances versus the effective thickness of gold film. The initial peak-to-peak amplitudes of the bare gratings are shown as a histogram in Figure 4.5(b). The inset shows the measured spectral changes of the pair of cladding modes used for the $18 \mathrm{~nm}$ thick film.

Theoretical model for complex refractive index of gold CVD films. In order to calculate the complex refractive index $\left(n_{f}-i k_{f}\right)$ of the gold NP films from the TFBG data, an effective medium approach is used, whereas an equivalent uniform film is used for the NP coating (note that we use the convention of a positive $k_{f}$ for optical loss in choosing the sign of the imaginary part in the expression for the complex refractive index). The thickness of the equivalent film is chosen to be equal to the average step height of the corresponding actual coatings. Figure 4.6 
shows this correspondence schematically. Once this is done, the real and imaginary parts of the propagation constant of the optical modes guided by the structure of Figure 4.6(b) can be calculated and compared to the experimental data. The mode propagation constant is equal to $2 \pi N_{\text {eff }} / \lambda$, where $\lambda$ is the wavelength of the light and $N_{\text {eff }}$ the mode "effective index" $\left(N_{e f f}=N_{e f f}^{r}-i N_{e f f}^{i}\right)$, i.e. the quantity that is monitored here. The aim of the simulation is to find the complex refractive index of the gold NPs film $\left(n_{f}-i k_{f}\right)$ that reproduces the spectral shifts shown in Figure 4.5, for each film thickness and for each mode polarization. Separate calculations for TE and TM modes allow the determination of any anisotropy (birefringence in $n_{f}$ or dichroism in $k_{f}$ ) in the deposited films.

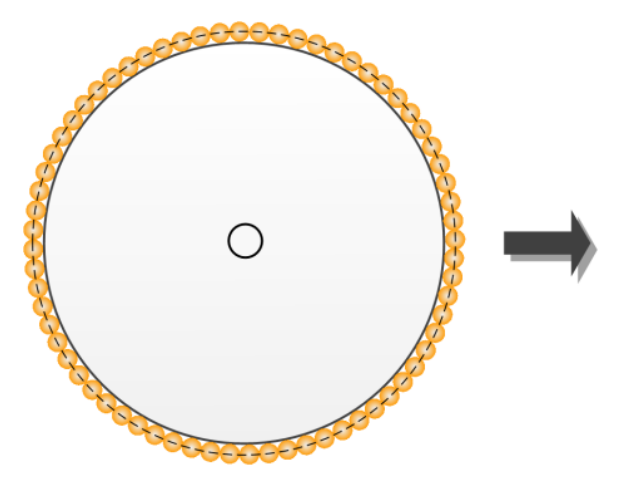

(a)

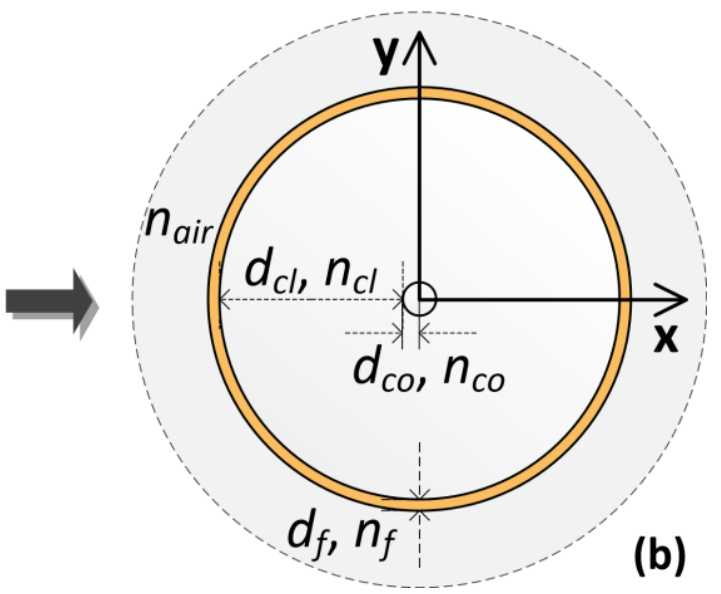

(b)

Fig. 4.6. Cross section of multi-layer model of optical fiber with gold NP film (dash line indicates the average thickness of the gold film) (a) and uniform gold film with equivalent thickness (b).

The data extraction algorithm proceeds as follows:

1 - Find the effective indices (real in this lossless case) of the pair of modes of the bare fiber that were used in the experiments, using the following formula for the real part of the effective index $N_{\text {eff }}^{r}$ [27],

$$
N_{e f f}^{r}=\lambda_{c l} \cos \theta / \Lambda-n_{c o}
$$


where $\lambda_{c l}$ is the wavelength of the cladding mode resonance, $\theta$ is the tilt angle of the grating planes, $\Lambda$ is the grating period, and $n_{c o}$ is the effective index of the core mode.

2 - Using the grating length (using $L=10 \mathrm{~mm}$ because the light goes through the $5 \mathrm{~mm}$ long grating twice in this reflective configuration) as a fixed parameter, find the coupling constant $\kappa$ of the mode from the initial peak-to-peak resonance transmission amplitude $T$ (corrected from the $\mathrm{dB}$ scale to linear units) using the following formula,

$$
\kappa=\tanh ^{-1}(\sqrt{1-T}) / L
$$

3 - Use a "mode solver" to calculate the modes of the structure shown in Figure 4.6(b) for the $d_{f}=0$ case and find the pair of modes with the value of $N_{e f f}^{r}$ found in part 1. A commercial complex mode solver based on vectorial finite differences was used for this purpose (FIMMWAVE, by Photon Design). The fiber parameters, that remain fixed throughout the process, are: the thickness (radius) of core, $d_{c o}=4.15 \mu \mathrm{m}$; the thickness of cladding, $d_{c l}=58.35$ $\mu \mathrm{m}$; the thickness of surrounding air layer is set as $17.5 \mu \mathrm{m}$; and the corresponding refractive indices of every layer at the wavelength of $1570 \mathrm{~nm}$ are $n_{c o}=1.45033, n_{c l}=1.44402$, and $n_{\text {air }}=$ 1.

4 - For each thickness and wavelength shift value reported in Figure 4.5(a), find the measured shift in $N_{\text {eff }}^{r}$ by equation 1.

5 - Similarly, from the change in $T$, determine the imaginary part of the effective index of the mode of the coated fiber at each thickness. The formula relating $N_{\text {eff }}^{i}$ to $\mathrm{T}$ is a little more complicated and we use a commercial grating solver for this purpose (Optigrating, by Optiwave).

6 - The calculated complex effective indices of the modes at each thickness are reported on Figure 4.7. The final step is run the mode solver again, with the added equivalent layer, and 
to look for values of $n_{f}$ and $k_{f}$ that reproduce the shifts in the complex effective index of the modes found in steps 4 and 5. The final results are plotted in Figure 4.8.

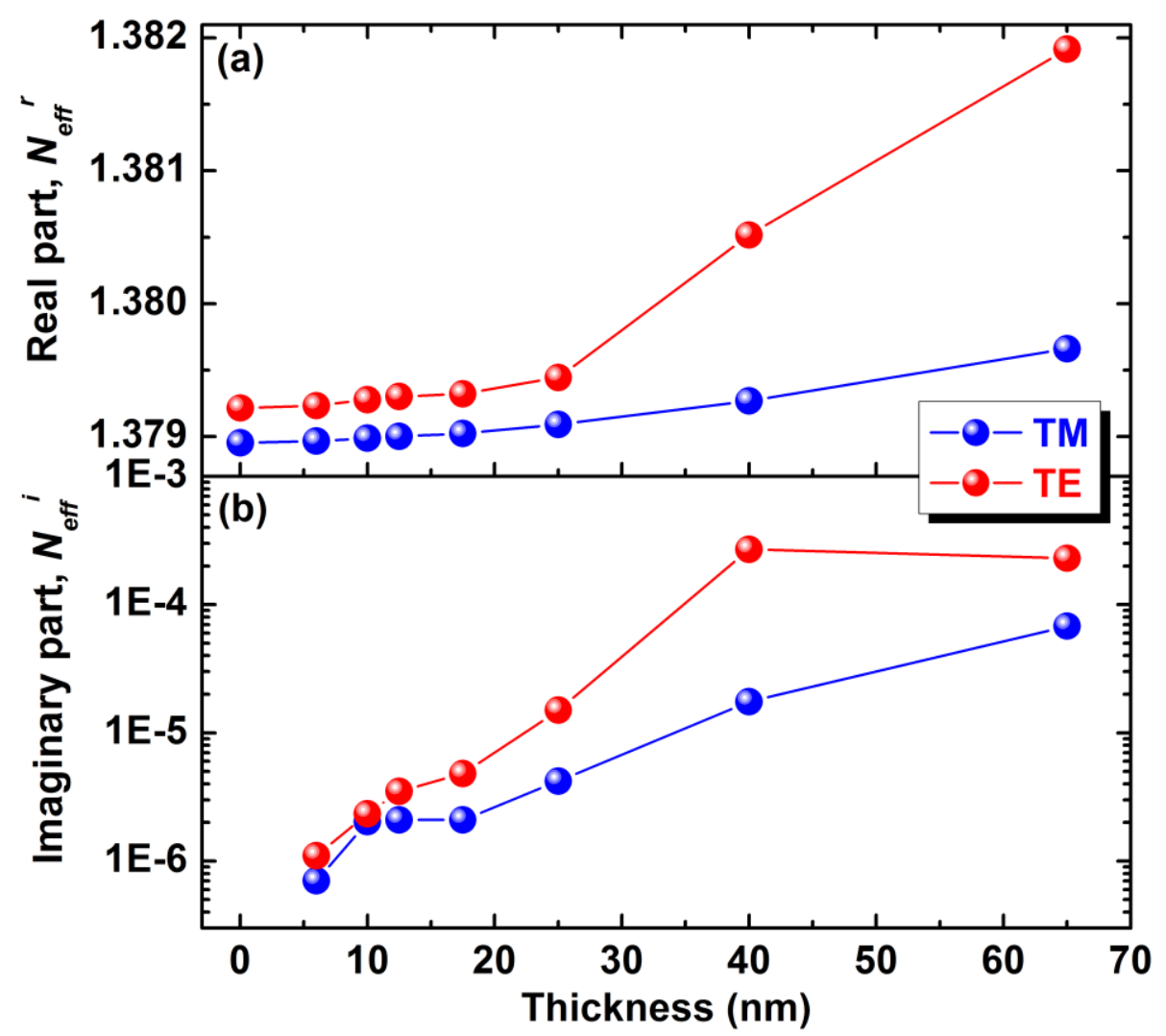

Fig. 4.7. Real (a) and imaginary (b) parts of the mode effective indices for the TE- and TM-polarized cladding modes measured in Figure 4.5, versus the thickness of the gold film. 


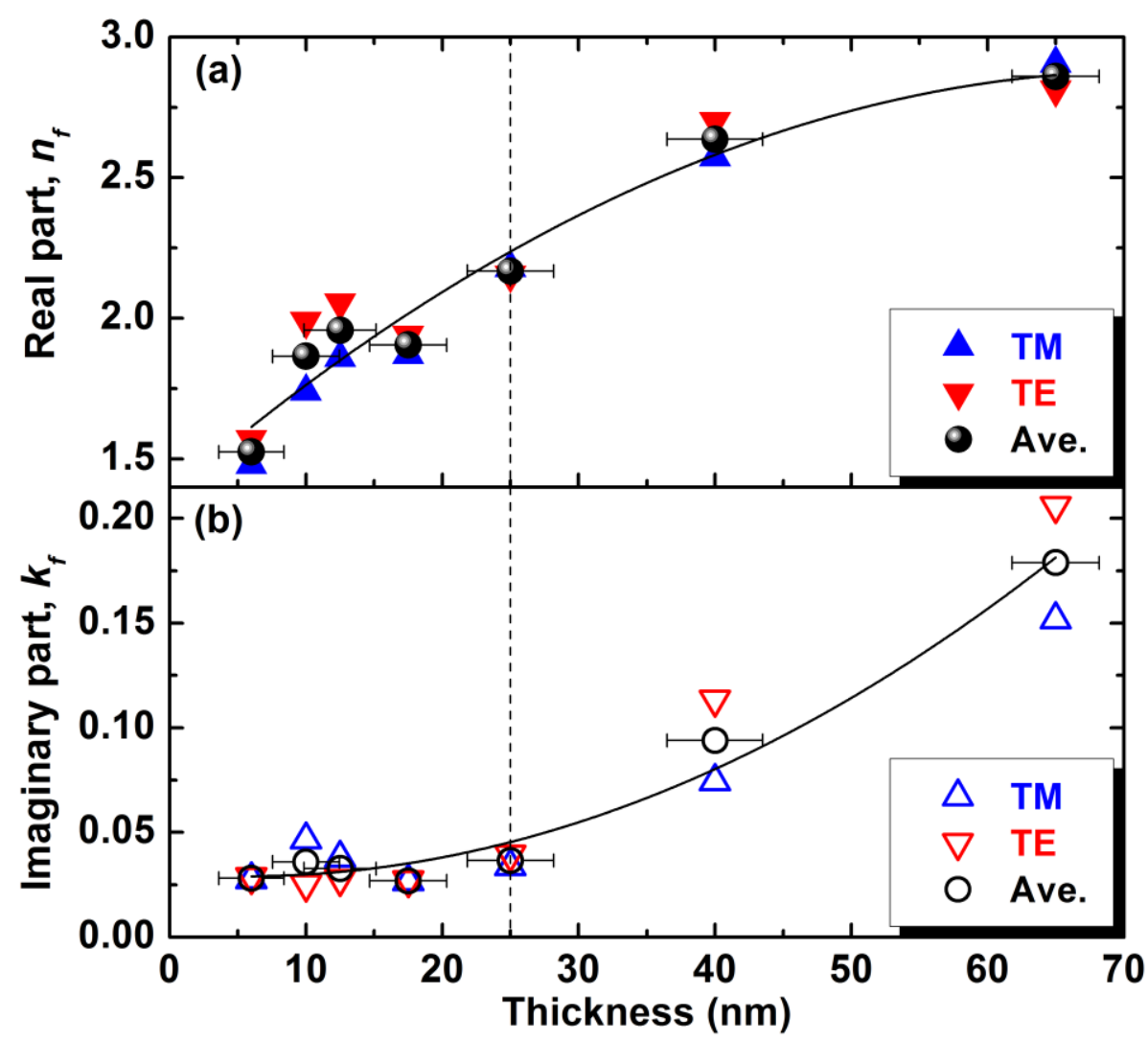

Fig. 4.8. Calculated real (a) and imaginary (b) parts of complex refractive indices of gold NP film in TEand TM-polarizations, and corresponding average values versus effective thickness. The dashed line at 25 $\mathrm{nm}$ represents the mean free path of electrons in gold. The horizontal error bar for each thickness is obtained from the standard deviation of the Gaussian fitting curve of the height histograms shown in Table 4.1.

\subsection{Discussions}

It is clear from Figure 4.7 that both the TE- and TM-polarized resonances shift to longer wavelengths for these thin gold films, but with a different shift magnitude, a difference that increases with thickness over the range shown here. If the coating refractive index was that of bulk gold (0.5-i10.9 according to [11] at these wavelengths), the wavelength shift should have been to shorter wavelengths because of the decrease in the refractive index at the cladding boundary. The overall mode loss also increases, which is expected from the addition of a lossy 
coating, by different values depending on polarization. However these measurements can be somewhat misleading as they not only depend on the complex refractive index of the added film but also on its thickness, as well as on the polarization dependence of the fiber waveguiding properties. Figure 4.8 reveals instead that the complex refractive index values for the film as probed by the TE and TM modes are essentially identical and therefore that the films are optically isotropic, apart from a weak increasing dichroism for thicknesses above $25 \mathrm{~nm}$. The line in Figure 4.8 follows the average complex refractive index calculated from the TE and TM mode probe results. Based on the statistically determined measurement uncertainty of $1 \mathrm{pm}$ in the central wavelength and of $5 \mathrm{e}-3 \mathrm{~dB}$ in the peak to peak amplitude of TFBG cladding mode resonances, the worst case error in the complex refractive index calculation was $\pm(0.03-10.005)$, obtained for the case of the $6 \mathrm{~nm}$ thick gold film. This final uncertainty is smaller than the size of the plotted points on Figure 4.8. The most striking feature of Figure 4.8 is that the complex refractive index measured here differs markedly from the bulk values for gold at these wavelengths. In particular, and this is a feature that has important practical consequences, the high value of the real part of the film index relative to the imaginary part means that the real part of the film's permittivity $\left(\varepsilon_{f}^{r}=n_{f}^{2}-k_{f}^{2}\right)$ is positive and therefore that these films cannot support the propagation of surface plasmon polaritons (SPP) (unlike gold films deposited by other methods including evaporation, sputtering, and electroless plating) [22]. Also, the films deposited by CVD are 100 times less lossy than bulk gold for thicknesses below $20 \mathrm{~nm}$, meaning that very little power is transferred from the fiber to the coatings.

These results also differ from those obtained for gold films of similar thicknesses deposited by other means. In particular, it has been shown that evaporated gold films have optical properties at $1550 \mathrm{~nm}$ wavelengths that were essentially identical to those of bulk gold, 
down to $20 \mathrm{~nm}$ thicknesses [21]. For thinner films down to $15 \mathrm{~nm}$ the imaginary part of the index decreases rapidly and the real part increases from 0.5 to 1.0 (but the real part of the permittivity remains negative). The deposition rate for these evaporated films was $3 \mathrm{~nm} / \mathrm{min}$. Another recent paper studied even thinner evaporated films (deposited at room temperature with a deposition rate of $12 \mathrm{~nm} / \mathrm{min}$ ) and showed that the transition from discontinuous (low loss, high real index) to continuous (bulk) behavior occurred for thicknesses between 2 and $10 \mathrm{~nm}$ (results obtained at visible wavelengths however) [23]. There is a large body of literature, dating back to at least 1950, which indicates that these differences can be attributed to the morphology of the gold films deposited by the various methods $[23,34]$ relative to that of the CVD gold films shown in Figure 4.4. It is clear that the CVD films do not have a tendency to morph from isolated NPs into continuous clusters of increasing size as the deposition progresses, as observed generally. The slow deposition rate (estimated to be near $1 \mathrm{~nm} / \mathrm{min}$ ) and high substrate temperature of $350{ }^{\circ} \mathrm{C}$ in this gold CVD process [35] yield a lower optical loss and larger (real) refractive index, due to the highly fragmented nature of the films which continue to grow via nucleation instead of aggregation. The behavior shown here was observed previously only for films deposited at very slow rates [36] or with high substrate temperatures $[37,38]$. It is interesting to note also the effective complex refractive index of films made up of very sparsely distributed gold NPs $(10 \%$ volume fractions for $10 \mathrm{~nm}$ diameter particles distributed randomly on a flat surface) was measured to lie near $(1.7-\mathrm{i} 0.1)$ at visible wavelengths [19], almost identical to what was obtained here for the much denser $11 \mathrm{~nm}$ thick films, albeit at infrared wavelengths. Finally, Figure 4.8 shows the beginning of a saturation of the growth in the real index, an increase in loss and the beginning of dichroism at a thickness of $25 \mathrm{~nm}$ (indicated by the vertical dashed line in the figure), which is corresponds to generally accepted mean free path of electrons in gold [23]. 
It thus appears that when the nanoparticulate films reach this thickness some paths form for electrons to transit between NPs, but very few.

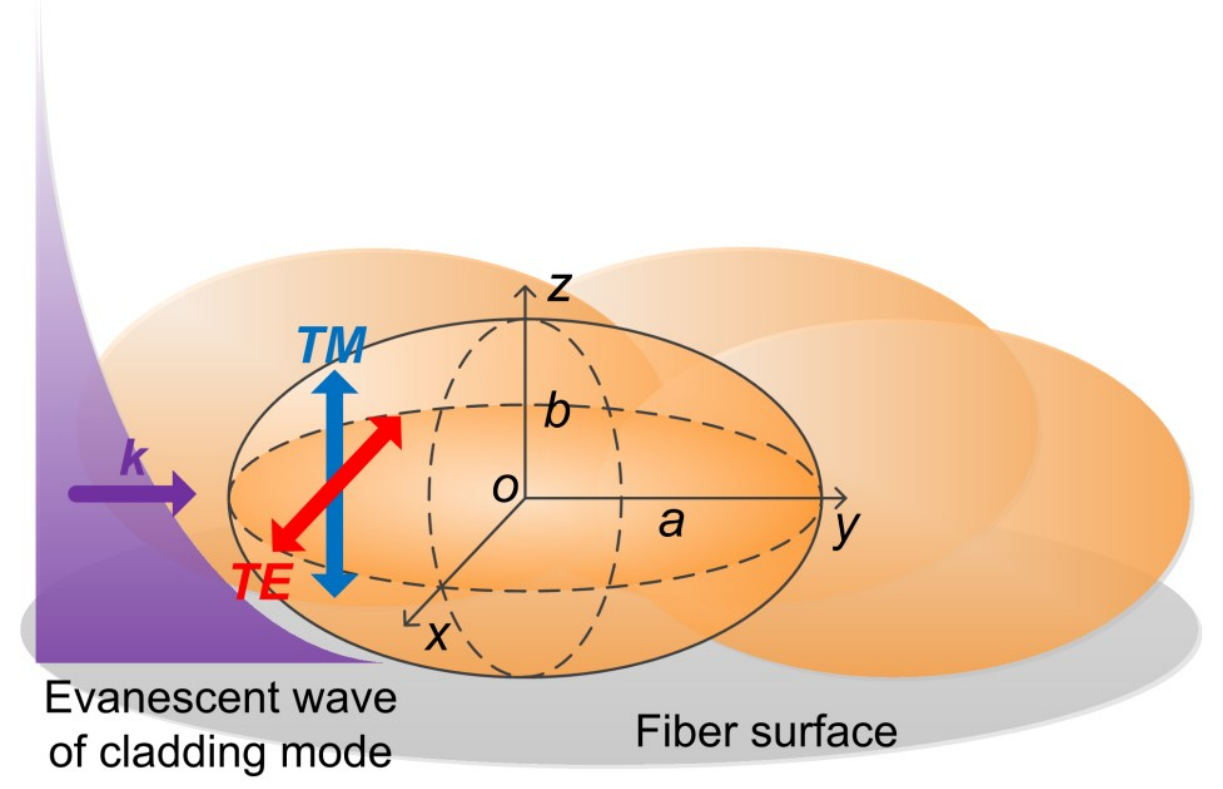

Fig. 4.9. Schematic diagram of spheroidal gold nanoparticles deposited on fiber surface (assuming that the fiber surface of small area $\left(\mu \mathrm{m}^{2}\right)$ is located in $x y$ plane), interacting with evanescent field of polarized cladding mode. The gold spheroids are oblate that the major axis $a$ and minor axis (polar axis) $b$ are parallel and perpendicular to $x y$ plane, respectively.

In order to further investigate the electromagnetic properties of the films produced, we now proceed with an evaluation of the inherent optical properties of the NPs by modelling the films using the Clausius-Mossotti model. For the most general case of anisotropic complex permittivity, it is assumed that the CVD gold film is composed of gold NPs with an oblate spheroid shape (according to the information provided in Table 4.1). Such NPs have different polarizabilities in the major and minor axes. Figure 4.9 illustrates how the evanescent field of TE and TM cladding modes propagates through these spheroids on the surface of the fiber. The major $(a)$ and minor $(b)$ axes of the oblate gold spheroids are aligned with the TE and TM 
electric fields, respectively. The generalized Clausius-Mossotti equation for a layer of metal spheroids embedded in an insulator material is [39],

$$
\frac{\varepsilon_{e f f}-\varepsilon_{i}}{L_{m}^{T M, T E} \varepsilon_{e f f}+\left(1-L_{m}^{T M, T E}\right) \varepsilon_{i}}=\phi \frac{\varepsilon_{m}-\varepsilon_{i}}{L_{m}^{T M, T E} \varepsilon_{m}+\left(1-L_{m}^{T M, T E}\right) \varepsilon_{i}}
$$

where $\varepsilon_{\text {eff }}$ is the effective permittivity of the mixture, $\varepsilon_{m}$ and $\varepsilon_{i}$ are the permittivities of metal and insulator, respectively, $L_{m}$ is the depolarization factor for TE or TM polarizations, and, $\phi$ is the volume fraction of metal material. The depolarization factors in the major $L_{m}^{T E}$ (TE-polarization) and minor $L_{m}^{T M}$ (TM-polarization) axes can be described as a function of the aspect ratio of the oblate metal spheroid [40],

$$
\begin{aligned}
& L_{m}^{T M}=\left(1+1.6 x+0.4 x^{2}\right)^{-1} \\
& L_{m}^{T E}=\left(1-L_{m}^{T M}\right) / 2
\end{aligned}
$$

where $x$ is the aspect ratio (b/a) of the oblate spheroid. Equations 3 and 4 can be used to relate the measured effective permittivity (refractive index) of the gold films for each effective thickness to the properties of the metal inclusions by substituting suitable parameters for the aspect ratio and volume fraction of the gold NPs. For the permittivity of the NPs, the bulk values for gold at this wavelength were used. Figure 4.10 shows the real and imaginary parts of the refractive index for the two polarization states calculated by fitting equation 3 to the real part of the index only (i.e. Figure 4.8a) with the best values for the metal fraction and aspect ratio. The Clausius-Mossotti model obviously fits very well, without having to use a modification of the bulk permittivity to account for the particles with diameters under $25 \mathrm{~nm}$ [16]. The metal volume fractions and aspect ratio used in the fit range from $30.5 \%$ to $68.3 \%$, and from 0.8 to 1.0 , respectively. The aspect ratios are consistent with those extracted from Table 4.1, while the 
volume fractions confirm that substantial air gaps remain at all thicknesses. Note that the volume fraction for closely packed spheres of any diameter is $74 \%$ [41].

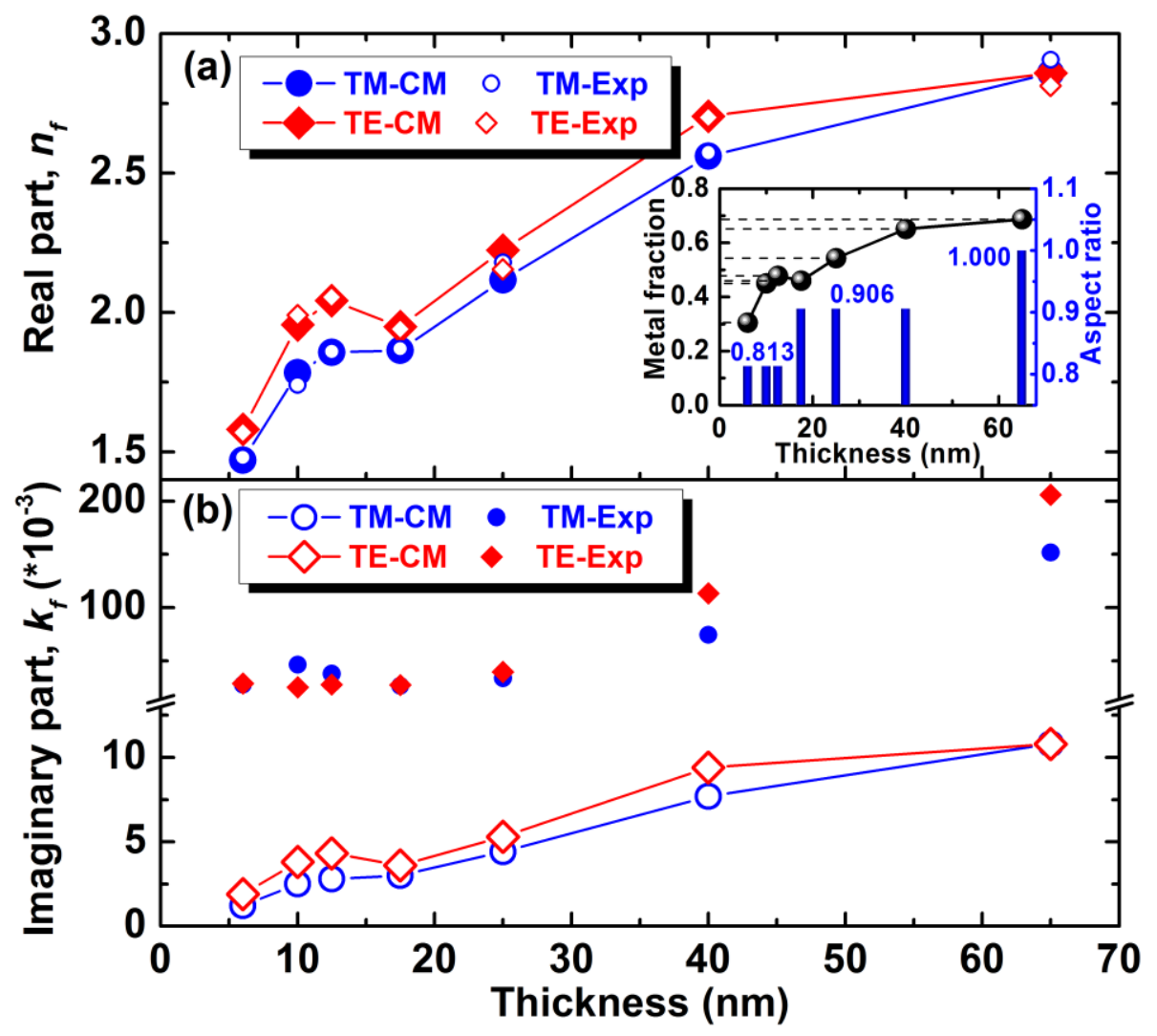

Fig. 4.10. Real (a) and imaginary (b) parts of gold nanoparticle films calculated by the modified ClausiusMossotti (CM) equation versus the measured effective thickness. The inset indicates the metal fraction and aspect ratio used in the CM model. The smaller hollow and solid scattered points (TM/TE-Exp) reproduce (from Figure 4.8) the real and imaginary part of the film index extracted from the measurements.

However, fitting the model to the real part of the permittivity results in a one order of magnitude underestimate of the imaginary part, and fails to reproduce the observed increased dichroism at thicknesses larger than $25 \mathrm{~nm}$. This is due to the fact that the imaginary part of the effective permittivity in the Clausius-Mossotti model comes solely from the imaginary part of the bulk permittivity of the metal inclusions, while the measurement of mode loss, from which 
the experimental effective medium properties are derived, include a strong scattering component and is therefore larger than modelled [29]. Since the fit indicates that the inclusions are nearly spherical, the imaginary part of the model fit is not expected to be anisotropic. Scattering however, from NP aggregates that are very thin in the out-of-plane direction but almost continuously connected in the plane, especially at increasing thicknesses, should be stronger (more lossy) for TE light than for TM light. This is what is observed. Another point of comparison is a more recent theoretical model for thin ( $7 \mathrm{~nm}$ and less) but continuous standalone gold films based on density functional theory (DFT) [42,43]. This DFT model predicts a sign change of the real part of the permittivity (from negative to positive) as well as a significant anisotropy between in-plane and out-of-plane components, when the interband band transitions of the gold electrons are added to the intraband transitions. However, the DFT model also does not reproduce our results quantitatively as it does not apply to NP aggregates in its theoretical framework.

\subsection{Conclusion}

In conclusion, the cladding modes of an optical fiber were used to investigate the complex refractive index of CVD gold coatings on the fiber cladding surface. The films were prepared from precursors with a high decomposition temperature above $300{ }^{\circ} \mathrm{C}$ and slow deposition rates near $1 \mathrm{~nm} / \mathrm{min}$. The AFM and SEM images of the gold CVD films with different thicknesses indicate that the films remain composed of nanoparticle aggregates with lateral sizes not exceeding $72 \mathrm{~nm}$, even for average film thicknesses up to $200 \mathrm{~nm}$. This kind of growth is of the Volmer-Weber type, i.e. island growth without coalescence into uniform layers [44]. The real and imaginary parts of the refractive index (and permittivity) of the gold nanoparticle films differ markedly from the bulk values at infrared wavelengths, as was often observed for films with 
thicknesses below $10 \mathrm{~nm}$. However in the case reported here, the anomalous values remain for thicknesses up to $65 \mathrm{~nm}$ and beyond, as in the case of evaporated gold films formed at very slow rates. The in-plane and out-of-plane components of the film permittivity were measured separately and shown to be essentially equal, apart from a small increasing dichroism at thicknesses larger than $25 \mathrm{~nm}$. The polarization-dependent refractive index of the metal-insulator mixture calculated by Clausius-Mosotti theory indicates that the gold CVD film consists of ellipsoidal particles with aspects ratios from 0.8 to 1.0 and air gaps corresponding to metal filling fractions increasing from $30 \%$ to $68 \%$ over the average thickness range from 6 to $65 \mathrm{~nm}$. A notable consequence of these results is that this CVD gold process cannot be used to coat optical fibers for the generation of surface plasmon polaritons $[45,46]$ because the films produced have a positive real permittivity. This is unfortunate because CVD produces inherently uniform and controllable film thicknesses around the fiber circumference, which helps to realize narrow and strong surface plasmon resonance devices. It remains to be determined whether post-processing of the films could increase the connectivity of the gold NPs to bring the permittivity of the resulting film closer to bulk values [36]. Finally it was demonstrated that the TFBG device can be used for the characterization at near infrared wavelengths of ultrathin metal films deposited by gas-phase processes, down to thicknesses below $10 \mathrm{~nm}$. The small TFBG probe can be inserted easily into deposition chambers to ensure that the film deposited on the probe is identical to that on adjacent substrates. It should be noted that for process temperatures below $200{ }^{\circ} \mathrm{C}$ the TFBG can be interrogated in real time during deposition and therefore provide film measurements as they grow [29]. 


\subsection{References}

1. Ciracì, C.; Hill, R. T.; Mock, J. J.; Urzhumov, Y.; Fernández-Domínguez, A. I.; Maier, S. A.; Pendry, J. B.; Chilkoti, A.; Smith, D. R. Probing the Ultimate Limits of Plasmonic Enhancement. Science 2012, 337, 1072-1074.

2. Chen, T.; Pourmand, M.; Feizpour, A.; Cushman, B.; Reinhard, B. M. Tailoring Plasmon Coupling in Self-Assembled One-Dimensional Au Nanoparticle Chains through Simultaneous Control of Size and Gap Separation. J. Phys. Chem. Lett. 2013, 4, 2147-2152.

3. Deeb, C.; Zhou, X.; Plain, J.; Wiederrecht, G. P.; Bachelot, R.; Russell, M.; Jain, P. K. Size Dependence of the Plasmonic Near-Field Measured via Single-Nanoparticle Photoimaging. J. Phys. Chem. C 2013, 117, 10669-10676.

4. Jägeler-Hoheisel, T.; Cordeiro, J.; Lecarme, O.; Cuche, A.; Girard, C.; Dujardin, E.; Peyrade, D.; Arbouet, A. Plasmonic Shaping in Gold Nanoparticle Three-Dimensional Assemblies. J. Phys. Chem. C 2013, 117, 23126-23132.

5. Yan, B.; Boriskina, S. V.; Reinhard, B. M. Optimizing Gold Nanoparticle Cluster Configurations (n $\leq$ 7) for Array Applications. J. Phys. Chem. C 2011, 115, 4578-4583.

6. Anker, J. N.; Hall, W. P.; Lyandres, O.; Shah, N. C.; Zhao, J.; Van Duyne, R. P. Biosensing with Plasmonic Nanosensors. Nat. Mater. 2008, 7, 442-453.

7. Mock, J.J.; Hill, R. T.; Tsai, Y.-J.; Chilkoti, A.; Smith, D. R. Probing Dynamically Tunable Localized Surface Plasmon Resonances of Film-Coupled Nanoparticles by Evanescent Wave Excitation. Nano Lett. 2012, 12, 1757-1764.

8. Sarkar, S.; Pradhan, M.; Sinha, A. K.; Basu, M.; Pal, T. Chelate Effect in Surface Enhanced Raman Scattering with Transition Metal Nanoparticles. J. Phys. Chem. Lett. 2010, 1, 439-444.

9. Baffou, G.; Quidant, R. Thermo-Plasmonics: Using Metallic Nanostructures as Nano-Sources of Heat. Laser Photon. Rev. 2013, 7, 171-187.

10. Cross, G. H. Fundamental Limit to the Use of Effective Medium Theories in Optics. Opt. Lett. 2013, 38, 3057-3060.

11. Johnson, P. B.; Christy, R. W. Optical Constants of the Noble Metals. Phys. Rev. B 1972, 6, 43704379.

12. Ordal, M. A.; Long, L. L.; Bell, R. J.; Bell, S. E.; Bell, R. R.; Alexander, Jr., R. W.; Ward, C. A. Optical Properties of the Metals Al, Co, Cu, Au, Fe, Pb, Ni, Pd, Pt, Ag, Ti, and $\mathrm{W}$ in the Infrared and Far Infrared. Appl. Opt. 1983, 22, 1099-1119.

13. Grosse, P.; Offermann, V. Analysis of Reflectance Data Using the Kramers-Kronig Relations. Appl. Phys. A 1991, 52, 138-144. 
14. Tu, J. J.; Homes, C. C.; Strongin, M. Optical Properties of Ultrathin Films: Evidence for a Dielectric Anomaly at The Insulator-to-Metal Transition. Phys. Rev. Lett. 2003, 90, 017402.

15. Kreibig, U.; Vollmer, M. Optical Properties of Metal Clusters; Springer: Berlin, Germany, 1995.

16. Kreibig, U.; Fragstein, C. V. The Limitation of Electron Mean Free Path in Small Silver Particles. $Z$. Physik 1969, 244, 307-323.

17. Sihvola, A. Electromagnetic Mixing Formulas and Applications; The Institution of Electrical Engineers: London, U.K., 1999.

18. Yamamoto, M.; Namioka, T. In situ Ellipsometric Study of Optical Properties of Ultrathin Films. Appl. Opt. 1992, 31, 1612-1621.

19. Chen, M.; Horn, R. G. Refractive Index of Sparse Layers of Adsorbed Gold Nanoparticles. J. Colloid Interface Sci. 2007, 315, 814-817.

20. Inagaki, T.; Goudonnet, J. P.; Royer, P.; Arakawa, E. T. Optical Properties of Silver Island Films in the Attenuated-Total-Reflection Geometry. Appl. Opt. 1986, 25, 3635-3639.

21. Lee, W.-J.; Kim, J.-E.; Park, H.-Y.; Park, S.; Kim, M.-S. Optical Constants of Evaporated Gold Films Measured by Surface Plasmon Resonance at Telecommunication Wavelengths. J. Appl. Phys. 2008, 103, 073713.

22. Homola, J. Electromagnetic Theory of Surface Plasmons. Springer Ser. Chem. Sens. Biosens. 2006, 4, $3-44$.

23. Wang, X.; Chen, K.-P.; Zhao, M.; Nolte, D. D. Refractive Index and Dielectric Constant Evolution of Ultra-Thin Gold From Clusters to Films. Opt. Express 2010, 18, 24859-24867.

24. White, I. M.; Fan, X. On the Performance Quantification of Resonant Refractive Index Sensors. Opt. Express 2008, 16, 1020-1028.

25. Zhu, H.; White, I. M.; Suter, J. D.; Zourob, M.; Fan, X. Integrated Refractive Index Optical Ring Resonator Detector for Capillary Electrophoresis. Anal. Chem. 2007, 79, 930-937.

26. Albert, J; Lepinay, S.; Caucheteur, C.; DeRosa, M. C. High Resolution Grating-Assisted Surface Plasmon Resonance Fiber Optic Aptasensor. Methods 2013, 63, 239-254.

27. Albert, J.; Shao, L.-Y.; Caucheteur, C. Tilted Fiber Bragg Grating Sensors. Laser Photon. Rev. 2013, 7, 83-108.

28. Shao, L.-Y.; Coyle, J. P.; Barry, S. T.; Albert, J. Anomalous Permittivity and Plasmon Resonances of Copper Nanoparticle Conformal Coatings on Optical Fibers. Opt. Mater. Express 2011, 1, 128-137.

29. Zhou, W.; Mandia, D. J.; Griffiths, M. B. E.; Bialiayeu, A.; Zhang, Y.; Gordon, P. G.; Barry, S. T.; Albert, J. Polarization-Dependent Properties of the Cladding Modes of a Single Mode Fiber Covered with Gold Nanoparticles. Opt. Express 2013, 21, 245-255. 
30. Mandia, D. J.; Griffiths, M. B. E.; Zhou, W.; Gordon, P. G.; Albert, J.; Barry, S. T. In Situ Deposition Monitoring by a Tilted Fiber Bragg Grating Optical Probe: Probing Nucleation in Chemical Vapour Deposition of Gold. Phys. Procedia 2013, 46, 12-20.

31. Alam, M. Z.; Albert, J. Selective Excitation of Radially and Azimuthally Polarized Optical Fiber Cladding Modes. J. Lightwave Technol. 2013, 31, 3167-3175.

32. Barry, S. T. Amidinates, Guanidinates and Iminopyrrolidinates: Understanding Precursor Thermolysis to Design a Better Ligand. Coord. Chem. Rev. 2013, 257, 3192-3201.

33. Othonos, A.; Kalli, K. Fiber Bragg Grating: Fundamentals and Applications in Telecommunications and Sensing; Artech House: London, U.K., 1999.

34. Ilanit, D.-M.; Zahava, B.; Neta, F.-G.; Alexander, V.; Israel, R. Ultrathin Gold Island Films on Silanized Glass. Morphology and Optical Properties. Chem. Mater. 2004, 16, 3476-3483.

35. Coyle, J. P.; Gordon, P. G.; Wells, A. P.; Mandia, D. J.; Sirianni, E. R.; Yap, G. P. A.; Barry, S. T. Thermally Robust Gold and Silver Iminopyrrolidinates for Chemical Vapour Deposition of Metal Films. Chem. Mater. 2013, 25, 4566-4573.

36. Sennett, R. S.; Scott, G. D. The Structure of Evaporated Metal and Their Optical Properties. J. Opt. Soc. Am. 1950, 40, 203-211.

37. Truong, V. V.; Scott, G. D. Optical Constants of Aggregated Gold Films. J. Opt. Soc. Am. 1976, 66, 124-131.

38. Truong, V. V.; Scott, G. D. Optical Properties of Aggregated Noble Metal Films. J. Opt. Soc. Am. 1977, 67, 502-510.

39. Cohen, R. W.; Cody, G. D.; Coutts, M. D.; Abeles, B. Optical Properties of Granular Silver and Gold Films. Phys. Rev. B 1973, 8, 3689-3701.

40. Jones, S. B.; Friedman, S. P. Particle Shape Effects on the Effective Permittivity of Anisotropic or Isotropic Media Consisting of Aligned or Randomly Oriented Ellipsoidal Particles. Water Resour. Res. 2000, 36, 2821-2833.

41. Ung, T.; Liz-Marzán, L. M.; Mulvaney, P. Optical Properties of Thin Films of Au@ $\mathrm{SiO}_{2}$ Particles. J. Phys. Chem. B 2001, 105, 3441-3452.

42. Laref, S.; Cao, J.; Asaduzzaman, A.; Runge, K.; Deymier, P.; Ziolkowski, R. W.; Miyawaki, M.; Muralidharan, K. Size-Dependent Permittivity and Intrinsic Optical Anisotropy of Nanometric Gold Thin Films: A Density Functional Theory Study. Opt. Express 2013, 21, 11827-11838.

43. Campbell, S. D.; Ziolkowski, R. W.; Cao, J.; Laref, S.; Muralidharan, K.; Deymier, P. Anisotropic Permittivity of Ultra-Thin Crystalline Au Films: Impacts on the Plasmonic Response of Metasurfaces. Appl. Phys. Lett. 2013, 103, 091106. 
44. Kolasinski, K. W. Surface Science: Foundations of Catalysis and Nanoscience; John Wiley \& Sons, Inc.: West Chester, PA, 2008.

45. Shevchenko, Y.; Albert, J. Plasmon Resonances in Gold-Coated Titled Fiber Bragg Gratings. Opt. Lett. 2007, 32, 211-213.

46. Shevchenko, Y.; Chen, C.; Dakka, M. A.; Albert, J. Polarization-Selective Grating Excitation of Plasmons in Cylindrical Optical Fibers. Opt. Lett. 2010, 35, 637-639. 


\section{Chapter 5: Anisotropic effective permittivity of an ultrathin}

\section{gold coating on optical fiber in air, water and saline}

\section{solutions $^{3}$}

The optical properties of an ultrathin discontinuous gold film in different dielectric surroundings are investigated experimentally by measuring the polarization-dependent wavelength shifts and amplitudes of the cladding mode resonances of a tilted fiber Bragg grating. The gold film was prepared by electron-beam evaporation and had an average thickness of $5.5 \mathrm{~nm}( \pm 1 \mathrm{~nm})$. Scanning electron imaging was used to determine that the film is actually

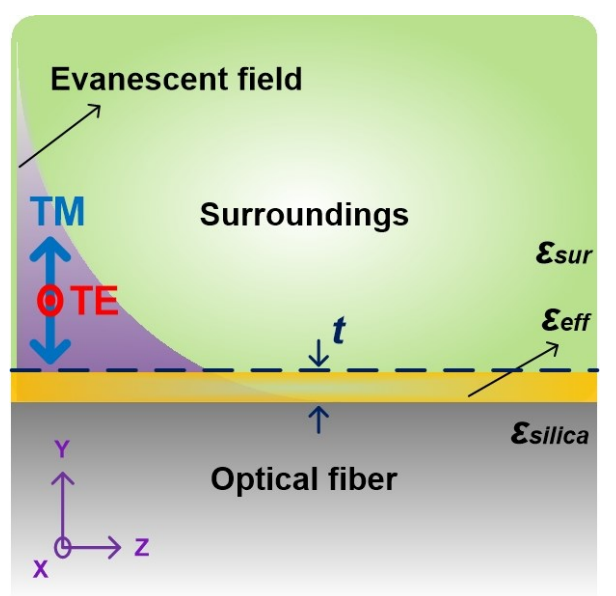
formed of individual particles with average lateral dimensions of $28 \mathrm{~nm}( \pm 8 \mathrm{~nm})$. The complex refractive indices of the equivalent uniform film in air at a wavelength of $1570 \mathrm{~nm}$ were calculated from the measurements to be $4.84-\mathrm{i} 0.74$ and 3.97-i0.85 for TM and TE polarizations respectively (compared to the value for bulk gold: 0.54-i10.9). Additionally, changes in the birefringence and dichroism of the films were measured as a function of the surrounding medium, in air, water and a saturated $\mathrm{NaCl}$ (salt) solution. These results show that the film has stronger dielectric behavior for TM light than for TE, a trend that increases with increasing surrounding index. Finally, the experimental results are compared to predictions from two widely used effective medium approximations, the generalized Maxwell-Garnett and Bruggeman theories for gold particles in a surrounding matrix. It is found that both of these methods fail to predict the observed behavior for the film considered.

\footnotetext{
${ }^{3}$ Reproduced with Permission from "W. Zhou, D. J. Mandia, S. T. Barry, and J. Albert, 'Anisotropic effective permittivity of an ultrathin gold coating on optical fiber in air, water, and saline solutions,' Optics Express 22(26), 31665-31676 (Dec. 2014)." Copyright 2014, Optical Society of America.

Author contributions: J.A. and W.Z. conceived the basic idea of the SRI-dependent effective permittivity of ultrathin gold coating on optical fiber. J.A. planned, supervised and supported the experimental implementation. W.Z. fabricated the TFBG probes, built the experimental setup, processed the experimental data, and performed the numerical simulations. D.J.M. performed the AFM imaging. W.Z., D.J.M., and J.A. wrote the manuscript. The project was organized and coordinated by J.A. and S.T.B.
} 


\subsection{Introduction}

Ultrathin gold films with thicknesses under $10 \mathrm{~nm}$ show size- and geometry-dependent features in their optical and plasmonic properties that are quite different from those of bulk material [1]. With decreasing thickness the thin gold films evolve morphologically from continuous to granular, during which a metal-to-insulator transition occurs [2,3]. The ultrathin gold aggregates can be represented by a single layer of oblate nanoparticles (NPs), and such layers have important applications as selective light absorbers [4,5] and many kinds of plasmonic devices $[6,7]$. Using the effective medium approximation (EMA) theories of the early 1900s [8], the complex effective permittivity of the aggregated gold films embedded in a homogeneous matrix (surrounding material) can be simulated. In the EMA models, the gold aggregates are represented by point dipoles embedded in the homogenous background, and the resulting effective permittivity of the mixture is dependent on the complex permittivities of the two constituents (gold and surrounding material), and on the volume fraction of gold. For experimental studies of thin gold films, spectroscopic ellipsometry is one of the most common measurement tools as it is highly sensitive to the optical properties of thin films [3], [9-11]. However, most of the previous work in this area was focused on measuring the thicknessdependent complex permittivity calculated from the ellipsometric angles to investigate the effect of various thin film growth mechanisms, including the impact of the shape and distribution of the NPs making up the ultrathin films. Comparatively few studies exist on the impact of the surrounding medium permittivity of these films $[12,13]$, and in such cases the gold NPs films are always embedded in solid dielectric material, making it impossible to study the same film under different surroundings. Moreover, the anisotropic optical properties of the ultrathin metal films caused by the nonspherical shape of their NP constituents limit the measurement accuracy of the 
conventional measurement methods, such as spectral reflectometry [3,14], ellipsometry $[9,10]$, and interferometric picometrology [15]. This is one of the reasons for which previous works often neglected the optical anisotropy in measurements of gold films with thicknesses spanning the insulator-to-metal transition. The purpose of the present paper is to investigate the impact of the surrounding medium on the anisotropic optical properties of highly disordered, morphologically complex gold NP aggregates with an average thickness of $5.5 \mathrm{~nm}$. This experimental study is carried out by probing the ultrathin film, deposited on the surface of the cladding of an optical fiber by two-step evaporation, using the polarized evanescent fields of the fiber cladding modes that are resonantly excited by a tilted fiber Bragg grating (TFBG) $[16,17]$. The anisotropic properties of the thin film are inferred from the changes in the amplitudes and wavelengths of the various cladding modes as the film coated fiber is exposed to various surroundings. Those results are then compared with EMA calculations based on measured NP shapes and bulk values of their permittivities, and the discrepancies observed are discussed in relation with the assumptions made by both the Maxwell-Garnett (M-G) and Bruggeman EMA models.

In more detail, due to the inclination of the grating planes of a TFBG along a specific direction, two families of polarized cladding modes with radial or azimuthal electric fields at the fiber cladding boundary can be selectively excited by probing with linearly polarized input coreguided light (as shown in Fig. 5.1) [16,17]. Figs. 5.1(b), 5.1(c), 5.1(d), and 5.1(d) illustrate the simulated electric fields of four typical polarized cladding modes with the same radial order (number of zeros in field amplitude as a function of radial coordinate) in standard single mode fiber for telecommunications. $\mathrm{TM}_{0 \mathrm{~m}}$ and $\mathrm{EH}_{\mathrm{nm}}$ modes are radially polarized and result from $\mathrm{P}$ polarized core mode input while the $\mathrm{TE}_{0 \mathrm{~m}}$ and $\mathrm{HE}_{\mathrm{nm}}$ modes are azimuthally polarized and result 
from S-polarized input. P- and S-polarizations refer to the orientation of the input electric field in the plane of incidence and out of the plane of incidence on the tilted grating fringes, respectively. In the following these mode families will be referred to as TM and TE to shorten the text. Due to the orthogonal electric fields at the interface of fiber cladding and surroundings, the TM- and TE-polarized cladding modes show distinct responses to surrounding refractive index (SRI) changes, but more strongly so when metal coatings or particles are present [18]-[20]. Therefore, the polarization-dependent properties of the cladding modes excited by TFBG can be used for surface plasmon resonance sensors [21-23] and measuring the anisotropic optical properties of thin metal coatings $[24,25]$. In this chapter, the SRI-dependent spectral responses of the two kinds of polarized cladding modes are used to investigate the effect of the surrounding permittivity on the anisotropic effective optical properties of an ultrathin gold NP film with a mean thickness of $5.5 \mathrm{~nm}$. Since the coated TFBG is both the substrate for the coating and the probe of its properties, successive measurements of a given coating in many surrounding environments can be very accurate as they are inherently independent of changes in either the film or the sensing probes, which remain unchanged. The complex effective permittivities of the coating are measured and compared with theoretical predictions based on both the generalized M-G and Bruggeman EMAs, wherein the input parameters are particle sizes and filling factors, extracted from scanning electron microscope (SEM) and atomic force microscope (AFM) image data, as well as literature values for the permittivity of bulk gold and of the surrounding media tested. 


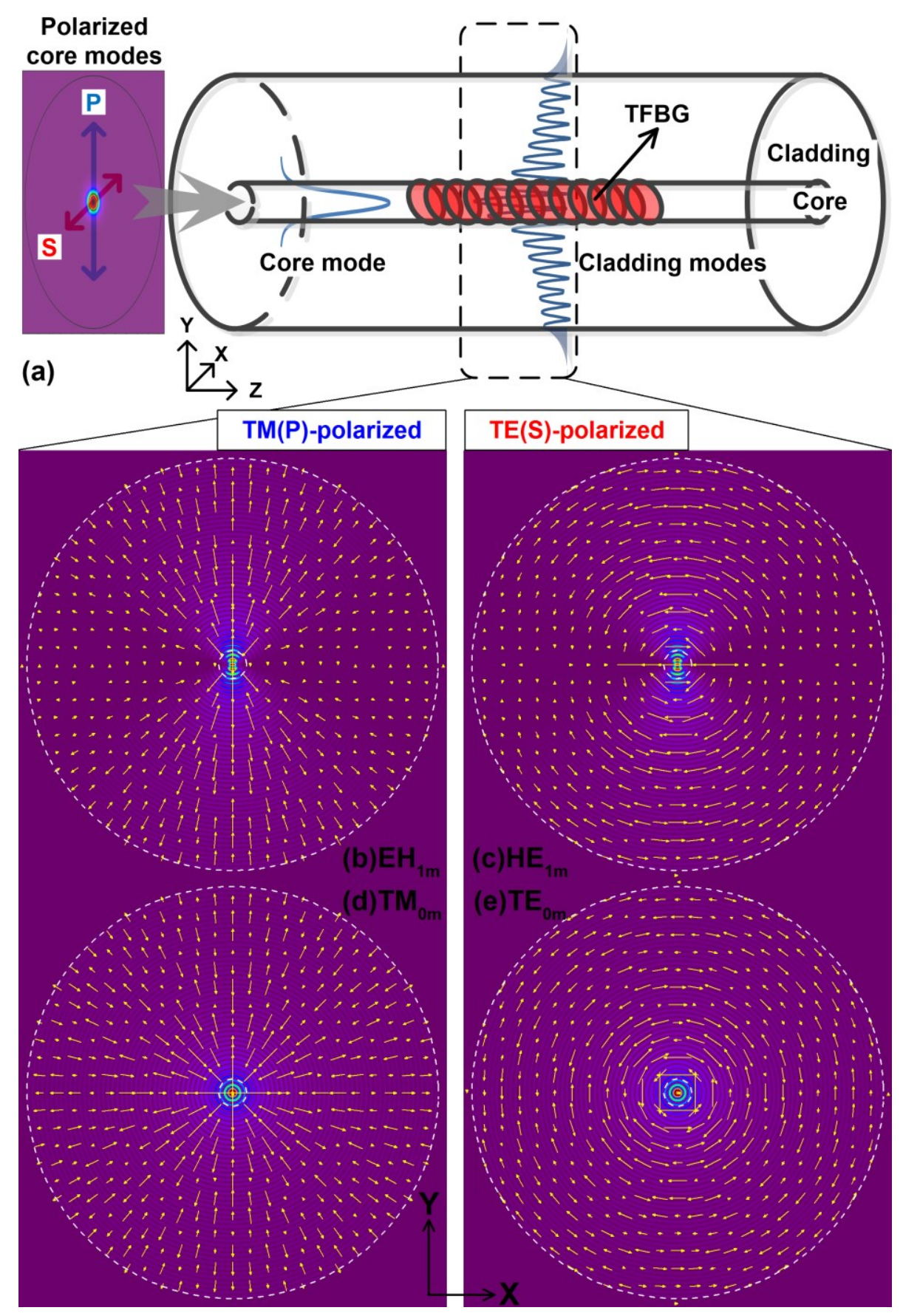

Fig. 5.1. (a) Schematic diagram of TFBG under $X$-(S-) and $Y$-(P-) polarized core modes input. Electric field distributions of four typically vectorial cladding modes ((b) $\mathrm{EH}_{1 \mathrm{~m}}$, (c) $\mathrm{HE}_{1 \mathrm{~m}}$, (d) $\mathrm{TM}_{0 \mathrm{~m}}$, and (e) $\mathrm{TE}_{0 \mathrm{~m}}$ ) coupled from the $X$-(S-) and $Y$-(P-) polarized core modes by TFBG, respectively. (Note that the grating planes are parallel to $X$ axis and tilted away from the $Y$ axis). The white broken curves indicate the core and cladding edges of optical fiber. 


\subsection{Sample fabrication and geometry}

The TFBG used in this work was written in hydrogen-loaded CORNING SMF-28 fiber with a pulsed $\mathrm{KrF}$ excimer laser using the phase-mask method [16]. The hydrogenation process of the optical fibers is as follows: a pressure of $15.2 \mathrm{MPa}$, a temperature of $20^{\circ} \mathrm{C}$, and a duration of 14 days. The length and the tilt angle were chosen at $10 \mathrm{~mm}$ and $10^{\circ}$ to excite a large number of high-order cladding modes with strong evanescent fields for thin coating measurements. The Bragg wavelength is around $1613 \mathrm{~nm}$, resulting from a phase mask period of $1114.8 \mathrm{~nm}$. After cleaning the TFBG cladding surface with piranha solution (a mixture of sulfuric acid and hydrogen peroxide), it was placed in an electron-beam physical vapor deposition system at room temperature and under vacuum ( $10^{-6}$ Torr). In order to have a relatively uniform gold coating around the fiber circumference, two gold deposition steps were conducted consecutively with the fiber being rotated by exactly $180^{\circ}$ between the two deposition runs using a custom designed fiber holding fixture. A relatively uniform gold film with a thickness of $\sim 5 \mathrm{~nm}$ was obtained under a deposition rate of $\sim 6 \mathrm{~nm} / \mathrm{min}$. The estimated thickness was obtained by measuring a witness sample (a 2-inch silicon wafer with a $30 \mathrm{~nm} \mathrm{SiO}_{2}$ thermal oxide buffer layer) that was placed next to the TFBG for one deposition run. We now proceed to describe physical measurements of the actual coating morphology (thickness and NP shapes and filling factor) on the witness sample.

Figure 5.2 shows SEM (a) and AFM (b) images which show that the $\mathrm{SiO}_{2}$ surface is completely covered by an irregular distribution of gold NPs. The evidence of small-scale coalescence of the aggregated gold NPs is already appearing for this thickness, resulting from the touching and merging of adjacent NPs. The quantitative analysis of the gold NP sizes and heights was carried out on the SEM and AFM images by using image processing software tools ImageJ 
and WSxM [26], respectively. The distributions of the lateral size (diameter) and height of the gold NPs film are shown in Figs. 5.2 (c) and 5.2(d), respectively. Based on the Gaussian fitting curves, the mean lateral size $d_{N P}$ and height $h_{N P}$ of the gold NPs are $28 \mathrm{~nm}$ with a standard deviation of $8 \mathrm{~nm}$ and $5.5 \mathrm{~nm}$ with a standard deviation of $1 \mathrm{~nm}$, respectively. The relatively large standard deviations in lateral size and height reflect the randomness of the cluster formation at these thicknesses, as can be clearly observed in Figs. 5.2(a) and 5.2(b). It is very clear that this $5.5 \mathrm{~nm}$ thick gold evaporated film can be regarded as a mono-layer of oblate gold nanospheroids. Also, the volume factor $f$ of the gold NPs is $68 \%( \pm 5 \%)$, as obtained from the SEM image using the ImageJ software.
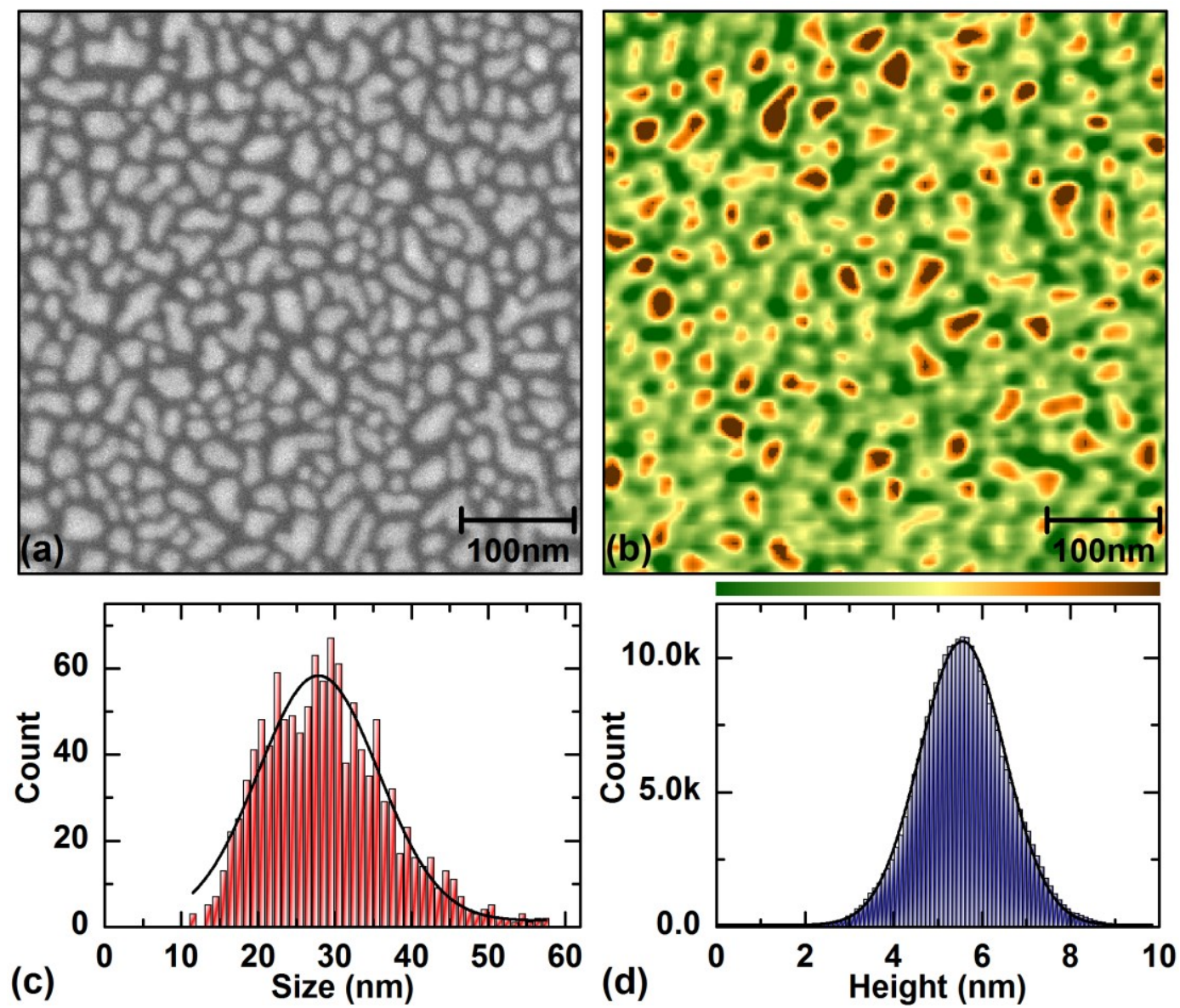

Fig. 5.2. SEM (a) and AFM (b) images of the gold NPs film over an area of $0.5 \times 0.5 \mu \mathrm{m}^{2}$ (with scale bar of $100 \mathrm{~nm}$ ). (c) Histogram of gold NP lateral sizes based on 1172 particles distributed over an area of 
approximately $1.3 \times 1.0 \mu \mathrm{m}^{2}$. (d) Height histogram of gold NPs film based on $\sim 2.62 \times 10^{5}$ extracted points from $1 \times 1 \mu \mathrm{m}^{2}$ AFM image.

\subsection{Experimental results}

Based on the experimental setup shown in Fig. 5.3(a) that includes a broadband source (BBS) (JDSU BBS1560), a polarization controller (PC) (JDSU PR2000), and an optical spectrum analyzer (OSA) (ANDO AQ6317B), the polarized spectra of TFBG under various SRIs can be measured [24]. Even though the nominal measurement resolution of this OSA is only $10 \mathrm{pm}$, the resonance lineshapes were fitted with an inverted Gaussian function over several measurement points and the statistically significant uncertainty with which an individual resonance can be measured is $\pm 2 \mathrm{pm}$ for the wavelength and $\pm 0.05 \mathrm{~dB}$ for the amplitude [16]. In this experiment, three different SRIs of $1,1.315-\mathrm{i} 1.21 \times 10^{-4}$ [27], and $1.360-\mathrm{i} 3.24 \times 10^{-5}$ [28] (at $1570 \mathrm{~nm}$ ) provided by air, deionized water, and a saturated $\mathrm{NaCl}$-water solution were applied on the TFBG sample. In order to investigate the coating with the same cladding guided mode (resonance) in the three different SRIs, a resonance near $1566.5 \mathrm{~nm}$ (measured in air) was chosen, as the cut-off wavelength for cladding mode guidance in the largest SRI is around $1565 \mathrm{~nm}$. The normalized spectra of the polarized cladding modes of the gold NPs-coated TFBG under the three surroundings is shown in Fig. 5.3(b). The spectra were adjusted to compensate for any temperature dependence by shifting them so that the core mode resonances for all the measurements (located near $1613 \mathrm{~nm}$ ) overlap perfectly [16]. All the spectra shown in Fig. 5.3 were obtained with the same TFBG, to eliminate any possible perturbation of the data by different underlying grating properties (it is not possible to fabricate exactly identical TFBGs by photosensitive processes). For comparison with the gold coated results, corresponding spectra of the same TFBG sample after etching of the gold coating are shown in Fig. 5.3(c). In Figs. 5.3(d) 
and 5.3(e), the floating columns indicate the extracted wavelength separations $\left(\lambda_{T E}-\lambda_{T M}\right)$ and peak-to-peak (P-P) amplitude differences $\left(\Delta A_{T E}-\Delta A_{T M}\right)$ between the TE- and TM-polarized cladding mode resonances under the three SRIs for both bare and coated TFBG samples.
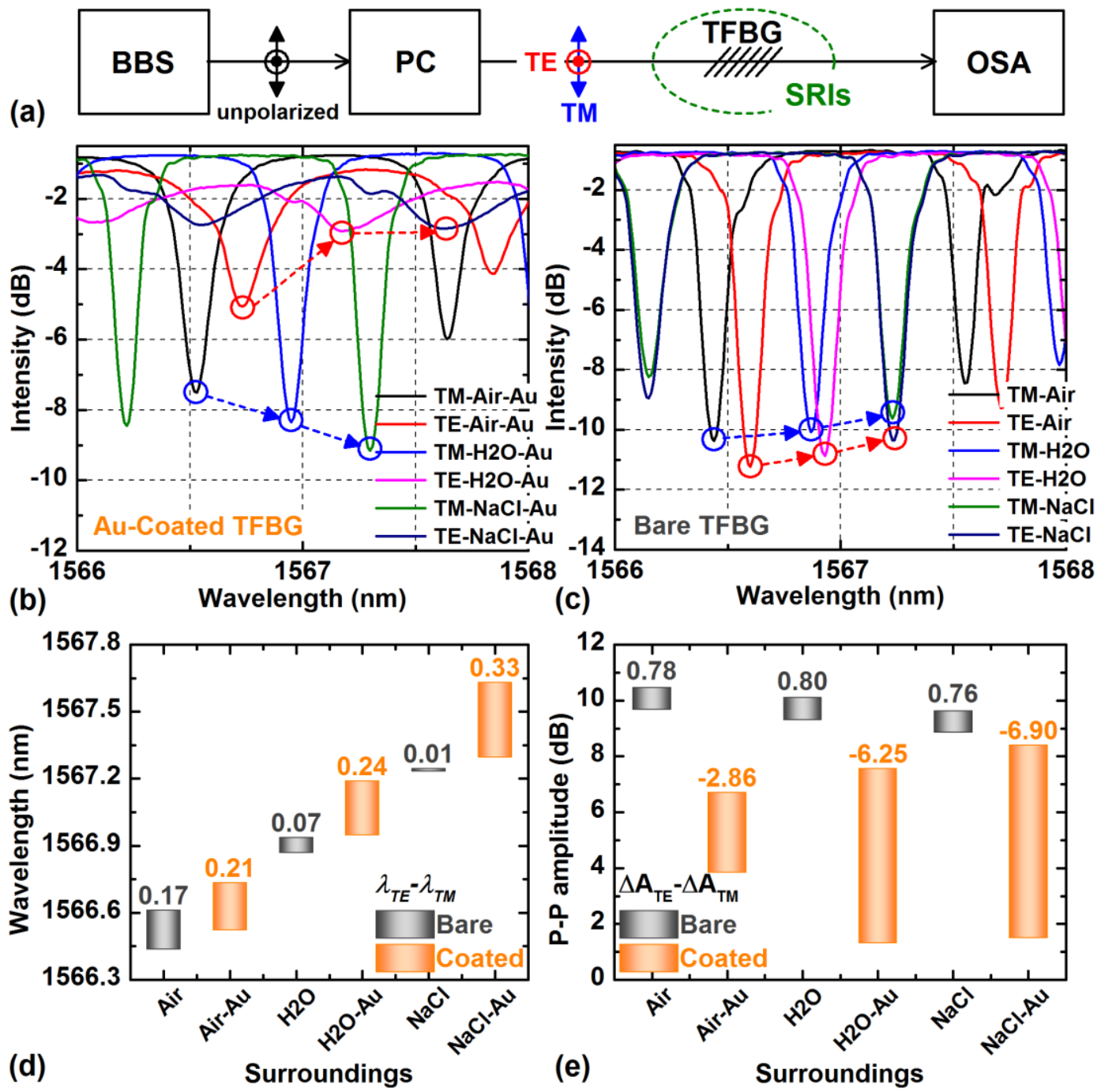

Fig. 5.3. (a) Schematic diagram of experimental setup. Temperature-calibrated spectra of the gold NPscoated (b) and the gold NPs-etched (bare) (c) TFBGs for TE- and TM-polarizations in air, DI water $\left(\mathrm{H}_{2} \mathrm{O}\right)$, and saturated $\mathrm{NaCl}$-water solution $(\mathrm{NaCl})$ surroundings. (The red and blue circles are marked at the positions of the TE- and TM-polarized cladding mode resonances, respectively.) Wavelength separations (d) and peak-to-peak amplitude differences (e) between the pair of polarized cladding modes for the both 
of coated and bare TFBGs under the three SRIs. Note that the signs of the P-P amplitude differences under the cases of bare and coated TFBGs are opposite.

It is obvious that the TE and TM cladding mode resonances both shift to longer wavelength under increasing SRI, as expected from standard waveguide theory, and roughly at the same average rate under these conditions (Fig. 5.3(d)). Also as expected, the wavelength difference between TE and TM resonances of the bare grating decreases from $0.173 \mathrm{~nm}$ to 0.013 $\mathrm{nm}$ with increasing SRI, indicating that the modes chosen approach their cut-off at the maximum SRI tested (since weakly guided and radiation modes no longer have polarization splitting [20]). For the gold coated TFBG on the other hand the SRI dependence of the wavelength shifts completely opposite: the wavelength separation for the same pair of resonances is larger in air $(0.210$ vs $0.173 \mathrm{~nm})$ and it further increases to $0.335 \mathrm{~nm}$ under the same changes of SRI as for the bare grating. So instead of reducing the polarization splitting, increasing the SRI makes the splitting larger when the TFBG is coated with the thin gold NP film.

With regards to the amplitudes of the resonances as the SRI rises, they decrease slowly for the bare TFBG as the modes approach cut off, and the amplitudes of the TM modes are slightly lower, due to the weaker confinement of those modes relative to the TE ones. Again the results for the grating with the gold coating are strikingly different. For the coated grating in air, the P-P amplitudes of all the modes are smaller than those of the bare grating, reflecting higher mode loss due to either absorption or scattering by the gold NPs. Upon increasing the SRI however, the amplitudes of the TM modes recover (they become larger, indicating lower mode loss) while those of TE modes continue to decrease. In fact, the overall spectral responses of the coated TE and TM modes indicate clearly that for such gold coatings, TE modes (with their electric fields polarized parallel to the film) are much more perturbed and are also more sensitive to further SRI changes. 


\subsection{Discussions}

In order to investigate the anisotropic optical properties of the gold NPs coating under the various SRIs, the effective indices of the polarized cladding modes have to be extracted from the spectral data. Then, those mode effective indices (along with the average coating thickness obtained by AFM) will be used to calculate the effective medium properties of the equivalent homogeneous layer that is added to the fiber waveguiding structure by the coating process. Fig. 5.4 illustrates the evanescent fields of the cladding modes with TE- or TM-polarization along the gold NPs-coated TFBG surface (a) and the corresponding waveguide (b) where the NPs have been replaced by an effective medium layer with homogeneous properties. Since the added layer has a complex permittivity, its effect on the effective indices of the TE and TM cladding modes is widely different [18,21-24].

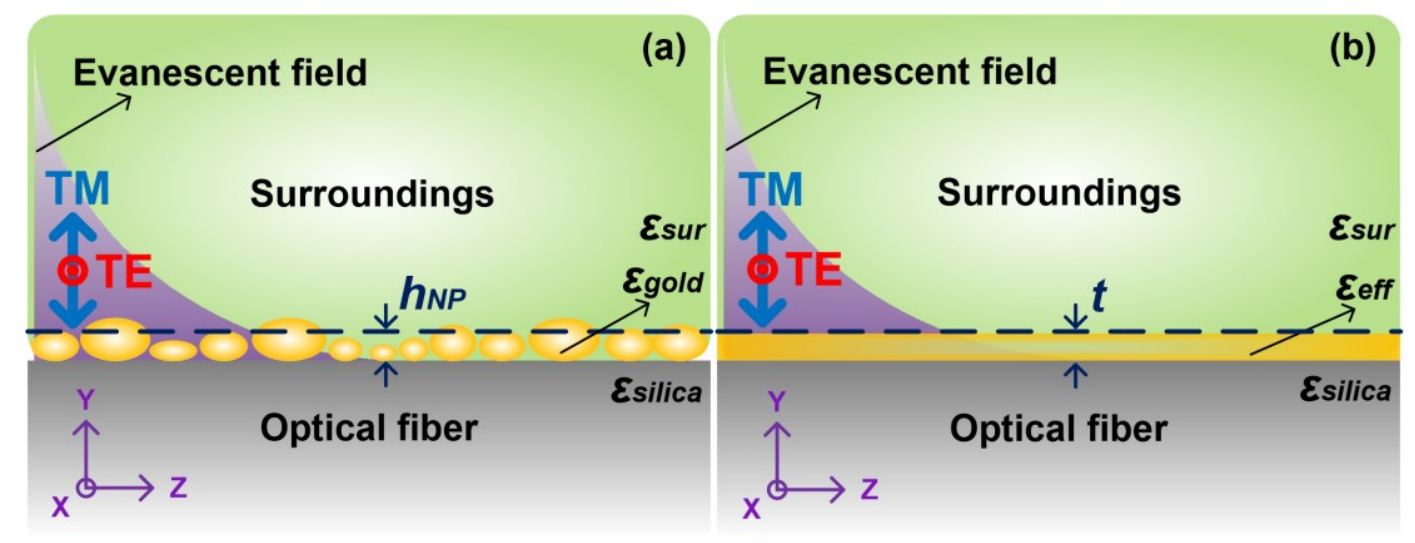

Fig. 5.4. 2D schematic diagrams of a mono-layer of oblate gold NPs (a) and effective medium layer that consists of gold and surrounding materials (b) coated on optical fiber surface under evanescent fields of TE- and TM-polarized cladding modes propagating along the $z$-axis. The thickness $t$ of the effective medium layer is equal to the average height $h_{N P}$ of the gold NP film measured from its AFM image. Note that the relative profiles of evanescent field and gold film shown above are not to scale, since the penetration depth of the cladding mode with effective index of $\sim 1.366$ in air is about $130 \mathrm{~nm}$. 
The real part of the effective index of a cladding mode can be obtained from the phase matching condition of the TFBG,

$$
N_{e f f}^{\text {clad }}=\lambda_{\text {clad }} \cos \theta / \Lambda-N_{e f f}^{\text {core }}
$$

where $\lambda_{\text {clad }}$ is the wavelength of the cladding mode resonance, $\theta$ is the tilt angle of the grating planes, $\Lambda$ is the grating period, and $N_{\text {eff }}^{\text {core }}$ is the effective index of the core mode $(\sim 1.447)$. The formula relating the imaginary part $K_{\text {eff }}^{\text {clad }}$ (the mode extinction coefficient) to the amplitude of the cladding mode attenuation in the transmission spectrum requires the computation of the coupling coefficient $\kappa$ between the incident core mode and the cladding mode of interest, given the known grating length and period, and the base fiber properties. The fundamental relationship between the coupling coefficient $\kappa$ and the $\mathrm{P}$-P transmission amplitude $\Delta A$ (converted from $\mathrm{dB}$ to linear scale) of the cladding mode can be written as,

$$
\kappa=\frac{\tanh ^{-1} \sqrt{1-\Delta A}}{L}
$$

where $L$ is the grating length $(10 \mathrm{~mm}$ ). This relationship is correct for the lossless case (no gold film). Once loss is introduced (the film), the coupling coefficient is unchanged to first order and the observed decrease in the P-P amplitude is due to the appearance of an imaginary part (i.e. absorption) in the cladding mode propagation constant. This imaginary part is found by matching experimental results to simulations of TFBGs with lossy modes, carried out with a numerical tool (Optigrating, by Optiwave) [24,25]. Figs. 5.5(a) and 5.5(b) show the real and imaginary parts of complex effective indices for TE and TM modes of the gold coated fiber extracted from the experimental mode resonances when the coated fiber was immersed in the three SRIs (Fig. 5.3(b)). The real part of the effective indices increases with SRI and the difference (TE-TM) increases from 0.00038 to 0.0006 . On the other hand, the calculation of the imaginary part of the 
effective index reveals that the extinction coefficient of the TE mode is enhanced from $1.51 \times 10^{-4}$ to $5.06 \times 10^{-4}$ in going from air to water, but decreases to $4.12 \times 10^{-4}$ in the salt-water solution. For the TM mode, extinction coefficient drops from $4.43 \times 10^{-5}$ in air to $7 \times 10^{-6}$ in the salt-water solution. It is important to note that the extinction coefficient for both TE and TM modes of the bare TFBG in the salt water solution is about $1 \times 10^{-6}$, which indicates absorption in the surrounding medium (due to its bulk extinction coefficient of $3.24 \times 10^{-5}$ ) contributes little to the extinction coefficient of the modes of the coated fiber.

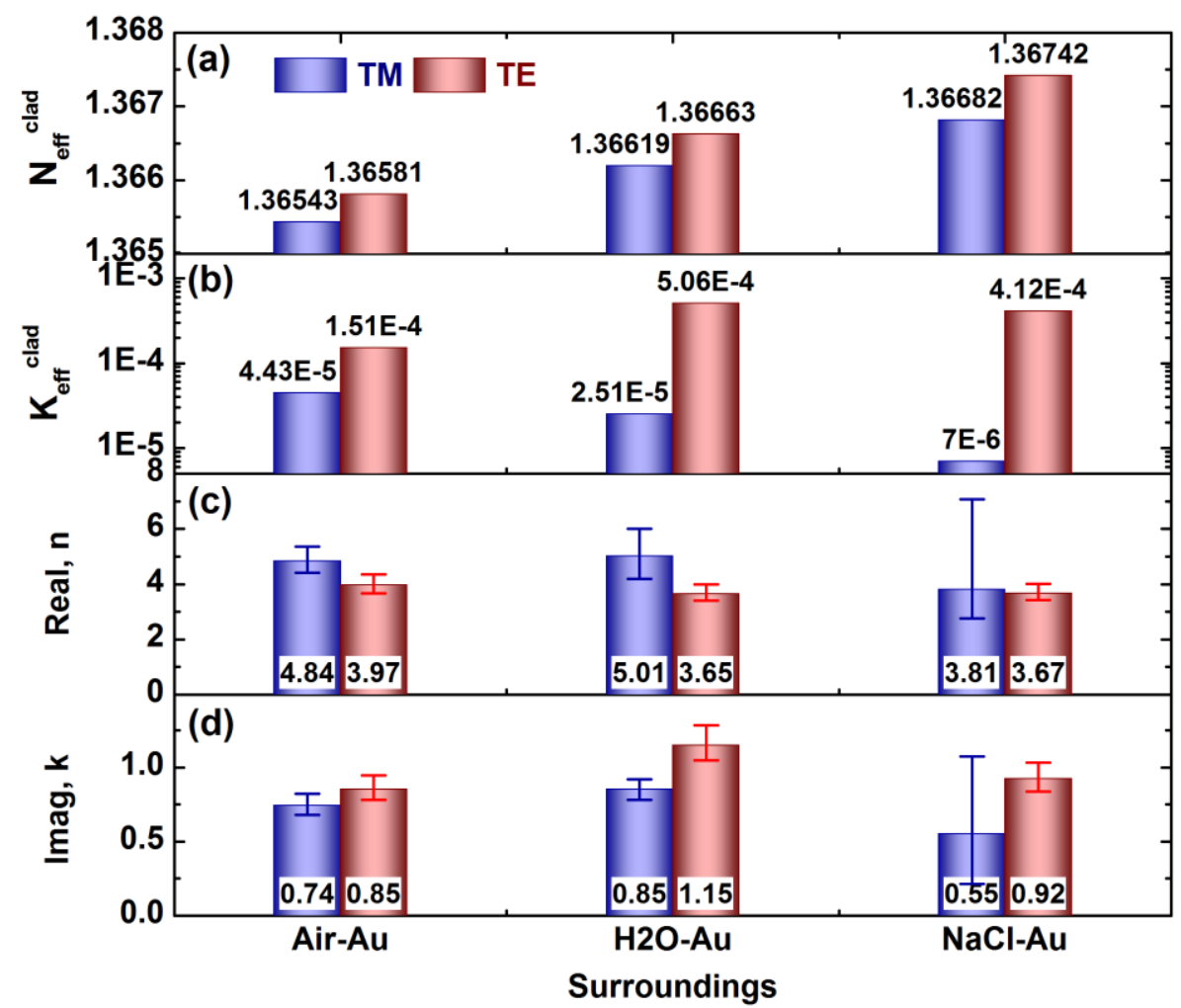

Fig. 5.5. Real (a) and imaginary (b) parts of the complex effective indices of the TE and TM cladding modes $\left(N_{\text {eff }}^{\text {clad }}-i K_{\text {eff }}^{\text {clad }}\right)$ under the three SRIs; Real (c) and imaginary (d) parts of complex refractive indices $(n-\mathrm{i} k)$ of the $5.5 \mathrm{~nm}$ thick effective homogeneous gold NPs film for TE and TM modes. The positive and negative errors of the complex refractive indices are evaluated from the thickness uncertainty of $\pm 1 \mathrm{~nm}$ in FIMMWAVE simulations. 
Now using the same optical fiber parameters (core and cladding) as those used in our previous paper [24], a thickness of $5.5 \mathrm{~nm}$ for the effective medium layer and the different SRIs as the outer layer, a four-layer optical fiber model solved with a vectorial finite differences method (FDM) complex mode solver (FIMMWAVE, by Photon Design) can be used to find the complex refractive index of the gold NP film $(n-\mathrm{i} k)$ that reproduces the measured mode effective indices $[24,25]$. While TE and TM modes always have different effective indices even for homogeneous, isotropic media [29], in the present case it was not possible to find a unique value of the complex film refractive index to yield the measured TE and TM modes: a film anisotropy had to be introduced to account for in-plane (TE) and out-of-plane (TM) electric field polarizations. The calculated effective medium complex refractive indices of the gold NPs film under the three SRIs at the cladding mode wavelength around $1570 \mathrm{~nm}$ are shown in Figs. 5.5(c) and 5.5(d). The different real and imaginary parts of the refractive indices for the TE and TM polarizations under the various surroundings indicate that the $5.5 \mathrm{~nm}$ gold NP film is optically anisotropic at this near infrared wavelength. Furthermore, the SRI dependence of complex refractive indices demonstrate that the gold NPs coating on TFBG surface can be regarded as a composite material layer consisting of gold NPs and a given surrounding medium. Compared with the complex refractive index of bulk gold (0.54-i10.9) [30], the calculated complex refractive indices of the gold NP layer has a 10 times higher real part and a 10 times lower imaginary part. In other words, the layer behaves much less as a metal than as a dielectric. This is consistent with the size-dependent optical properties of thin gold films investigated in other papers $[9-11,15]$, where the metallic character of gold is replaced with dielectric behavior with decreasing thickness, due to the confinement of free electrons caused by the interruption of percolation paths inside ultrathin metal films. Due to the electron mean free path of $25 \mathrm{~nm}$ [15], 
electrons are always confined in the gold NPs with an average thickness of $5.5 \mathrm{~nm}$ in the out-ofplane direction, suggesting dielectric-like optical properties for the TM-polarization. But the small-scale coalescence of the aggregated gold NPs with average lateral size of $28 \mathrm{~nm}$ weakens the localization effect of electrons in the in-plane direction, which results in the more metallic complex refractive index of the gold NPs film for the TE-polarization (lower real part and higher imaginary part). For quantitative comparisons, the measured real parts of the complex permittivities $\left(n^{2}-k^{2}\right)$ of the $5.3 \mathrm{~nm}$ and $6.2 \mathrm{~nm}$ thick gold evaporated films were around 25 and 10 at $1570 \mathrm{~nm}(0.79 \mathrm{eV})$ in air [3], respectively. From Figs. 5.5(c) and 5.5(d), the corresponding real parts of the anisotropic complex permittivities of the $5.5 \mathrm{~nm}$ gold film in air are 22.8 and 15 for the TM and TE polarizations, respectively, which are included within the above reported values. Moreover, the estimated complex refractive indices of the gold evaporated films with "nominal" thicknesses of $4 \mathrm{~nm}$ and $5 \mathrm{~nm}$ were found to be about 5-i1.5 and 4.5-i2.5 at $1570 \mathrm{~nm}$ in air [10], i.e. a similar real part as the one obtained here, but a much larger imaginary part. The origin of this discrepancy is that the gold films reported in [10] had larger-scale coalescence of the aggregated gold NPs than ours, which allowed more absorptive loss by Joule heating. The more connected gold films with lower thicknesses in [10] are probably caused by the difference between the mass-equivalent thickness and the effective thickness of the discontinuous gold films, which are correlated with each other by the filling factor (i.e. the mass-equivalent thickness of our gold NP film could be $\sim 3.7 \mathrm{~nm}(5.5 \times 68 \%))$ [3,31]. Moreover, previous work on thin gold films $(4-8 \mathrm{~nm})$ used a quasi-homogeneous isotropic effective medium material for investigating their optical properties in visible and infrared wavelength regions [10,15]. Here we provide further observations of the anisotropy of these films and demonstrate that directional 
depolarization effect in oblate gold NPs $[11,13,24]$ and the resulting anisotropic permittivity of ultrathin gold material [32,33], yields a measurable optical birefringence and dichroism.

For further investigating the anisotropic optical properties of the gold NPs coating under various SRIs, we now compare our measured results with theoretical calculations of the complex permittivity of an effective medium layer composed of gold NPs (using the bulk complex permittivity of gold at these wavelengths) and surrounding media. Two effective medium approximations (EMA) will be used, one based on the generalized M-G formula [34] and the other on the generalized Bruggeman formula [35]. From the SEM and AFM images shown in Fig. 5.2, the gold NPs can be represented as oblate gold spheroids embedded in the surrounding medium, where the minor axes of all oblate spheroids are perpendicular to the optical fiber surface. Thus, the generalized M-G and Bruggeman formulas can be respectively expressed as $[34,35]$,

$$
\begin{aligned}
& \frac{\varepsilon_{e f f}-\varepsilon_{e}}{\varepsilon_{e}+N_{T M, T E}\left(\varepsilon_{e f f}-\varepsilon_{e}\right)}=f \frac{\varepsilon_{i}-\varepsilon_{e}}{\varepsilon_{e}+N_{T M, T E}\left(\varepsilon_{i}-\varepsilon_{e}\right)} \\
& (1-f) \frac{\varepsilon_{e f f}-\varepsilon_{e}}{\varepsilon_{e f f}+N_{T M, T E}\left(\varepsilon_{e}-\varepsilon_{e f f}\right)}=f \frac{\varepsilon_{i}-\varepsilon_{e f f}}{\varepsilon_{e f f}+N_{T M, T E}\left(\varepsilon_{i}-\varepsilon_{e f f}\right)}
\end{aligned}
$$

where $\varepsilon_{\text {eff }}$ is the effective permittivity of the effective medium layer, $\varepsilon_{i}$ and $\varepsilon_{e}$ are the permittivities of inclusion (gold) and surroundings, respectively, $N_{T M, T E}$ is the depolarization factor for TM or TE-polarization, and $f$ is the volume factor of the gold material. For the oblate gold spheroids, the depolarization factors of the TM- and TE-polarizations can be defined as a function of the aspect ratio $x$ of the oblate spheroid [36],

$$
\begin{aligned}
& N_{T M}=\left(1+1.6 x+0.4 x^{2}\right)^{-1} \\
& N_{T E}=\left(1-N_{T M}\right) / 2
\end{aligned}
$$


where $N_{T M}$ and $N_{T E}$ are 0.752 and 0.124 for our structure, based on the following expression for $x=h_{N P} / d_{N P}$. With Eqs. (3) and (4), the calculated complex refractive indices of the effective medium coating under the three SRIs for the out-of-plane (TM) and in-plane (TE) directions are shown in Fig. 5.6, where the corresponding results obtained from the experimental data are also indicated.
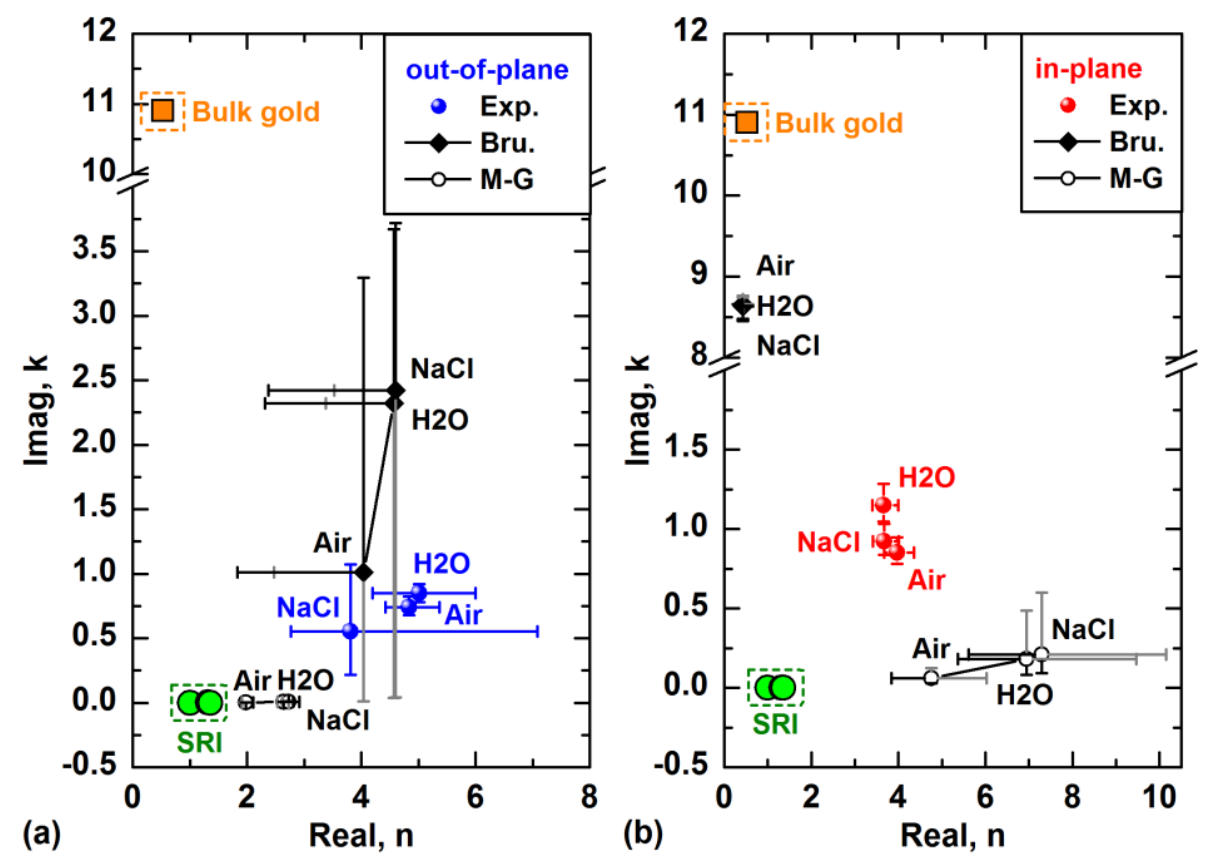

Fig. 5.6. Complex refractive indices of the effective medium coating of the three surroundings for (a) outof-plane (TM) and (b) in-plane (TE) directions calculated by M-G and Bruggeman EMAs, compared with the experimentally obtained results and the bulk permittivities of the medium constituents (gold and SRI). The gray and black error bars indicate the deviations of complex refractive indices caused by the minimum aspect ratio $x$ of $4.5 / 36$ and the maximum $x$ of $6.5 / 20$ in EMA models, respectively, based on the average height of $5.5 \mathrm{~nm}( \pm 1 \mathrm{~nm})$ and the average lateral dimensions of $28 \mathrm{~nm}( \pm 8 \mathrm{~nm})$ of the gold NPs. Note that the both of gray and black error bars show in negative direction for the real parts of Bruggeman EMA in Fig. 5.6(a).

Based on these comparisons of the three groups of complex refractive indices, some general trends can be observed. As expected from the EMA of metal particles in dielectric media, 
the large increase in the real part of the index of the film relative to that of its constituents is relatively well modeled, with the notable exception of the Bruggeman model for the in-plane (TE) polarization. In this latter case the model predicts a complex index that is very close to that of bulk gold (regardless of SRI), while the experimental data retains a strong dielectric behavior. It is as if the EMA layer was acting as a continuous metal barrier for light in the cladding and shielding it completely from the surroundings, which is not the case according to the experimental data but which does occur for thicker films [19]. The other observation is that the M-G EMA systematically underestimates the coating loss $(k)$, as expected in this case [37], while the Bruggeman model does the opposite (overestimates). An underestimated EMA value for loss is expected because the experimental values for loss come from two contributions in our experiments: absorption by gold NPs and scattering of the evanescent fields of cladding guided modes. Since the EMA approach does not include the effect of scattering, it was expected that those models would underestimate $k$ relative to the experiments. It is clearly not the case for the Bruggeman EMA which again reflects its tendency to overestimate the loss due to absorption (i.e. the metallic character of the ultrathin coating with such aspect ratio of its constituent NPs). In fact, the applicability of the various EMAs to the different steps of growing gold NPs film deposited on glass that was described in [38] indicates that the $M-G$ formula is quite suitable for sparsely distributed gold NP films while the Bruggeman formula is applicable when there is large-scale coalescence in the gold film growth. It looks from the results presented here that for intermediate levels of coalescence of ultrathin gold NP films, both EMA formulas fail in reproducing the experimental data. Some similar failures of the Bruggeman EMA were also observed in fitting the measured complex conductivities (directly related to complex permittivity) and optical transmittance at far-infrared frequency for gold films around the insulator-to-metal 
transition $[39,40]$. Our results for the anisotropic complex refractive index show that this gold NP film at a thickness of $5.5 \mathrm{~nm}$ is thicker than the critical value for the maximum dielectric constant, in agreement with measured critical thicknesses between $5.3 \mathrm{~nm}$ and $6.2 \mathrm{~nm}$ for light at a wavelength of $1570 \mathrm{~nm}(0.79 \mathrm{eV})$ [3]. The slightly weaker insulating properties of the gold NPs film for the in-plane polarization (TE) are understandable since the dipole coupling between the gold NPs should occur first for in-plane polarization and help the gold film tend toward metallic material as the film grows [11]. However, the significant differences in the birefringence and absolute values of the measured effective medium results as a function of SRI are unexpected and may reveal new and important effects that occur near the insulator-to-metal transition of gold at these thicknesses and wavelengths.

\subsection{Conclusion}

In conclusion, the SRI-dependent effective refractive indices of the anisotropic $5.5 \mathrm{~nm}$ gold NPs film deposited by gold evaporation method were investigated with the polarized cladding modes excited by a TFBG. The complex refractive indices for modes polarized in the in-plane (TE) and out-of-plane (TM) directions, and the corresponding effective thin coating parameters were extracted from the measured polarization-dependent wavelength shifts and amplitude changes of the cladding modes around $1570 \mathrm{~nm}$ with help from simulations carried out with an accurate four-layer complex mode solver. For the extracted complex refractive indices of the gold NPs film embedded in different media, the results show that the real parts are 10 times larger than for bulk gold while the imaginary parts of the film are 10 times smaller. Such ultrathin gold films with only small scale coalescence have therefore a strong dielectric nature. It is worth noting that for the gold NP sizes obtained here, the localized surface plasmon resonance (LSPR) wavelength is near $750 \mathrm{~nm}$, i.e. well outside of our measurement spectral range. While some of the NPs have 
larger sizes and hence longer LSPR wavelengths, the randomness of the sizes would smear out any significant impact. Therefore no plasmonic effect is expected to come into play. The slightly weaker insulating optical properties of the gold NPs film for the TE-polarization than those for TM-polarization suggest the proximity of an insulator-to-metal transition and a critical thickness lower than $5.5 \mathrm{~nm}$ at $1570 \mathrm{~nm}$, for the deposition conditions used. Moreover, comparing the experimental results with predictions from the generalized M-G and Bruggeman EMAs, it was revealed that these effective medium models fail to correctly account for the properties of the NP films at such thickness for near infrared wavelengths. The EMA models also do not correctly account for the effect of augmenting the surrounding medium permittivity on the birefringence and dichroism of the gold NPs film. In view of the increasing importance of thin metal coatings in plasmon-assisted sensing and in photovoltaic devices, the polarization-resolved, high sensitivity measurements reported here show that ultrathin, partially connected metal films have anisotropic effective complex permittivities that are not well modelled by currently accepted EMA theories that are based on bulk metal properties with shape and density factors alone. It was also demonstrated clearly that the evolution of the equivalent film properties with SRI presents some anomalous features that are not consistent with our understanding of the effect of thin perturbations on guided modes, especially in the important case of saline solutions.

\subsection{References}

1. U. Kreibig, and M. Vollmer, Optical Properties of Metal Clusters (Springer, 1995), Chap. 4.1.2.

2. J. J. Tu, C. C. Homes, and M. Strongin, "Optical properties of ultrathin films: evidence for a dielectric anomaly at the insulator-to-metal transition," Phys. Rev. Lett. 90(1), 017402 (2003).

3. M. Hövel, B. Gompf, and M. Dressel, "Dielectric properties of ultrathin metal films around the percolation threshold," Phys. Rev. B 81(3), 035402 (2010).

4. M. Svedendahl, P. Johansson, and M. Käll, "Complete light annihilation in an ultrathin layer of gold nanoparticles," Nano Lett. 13(7), 3053-3058 (2013). 
5. M. Yan, J. Dai, and M. Qiu, "Lithography-free broadband visible light absorber based on a monolayer of gold nanoparticles," J. Opt. 16(2), 025002 (2014).

6. N. Liu, M. Mesch, T. Weiss, M. Hentschel, and H. Giessen, "Infrared perfect absorber and its application as plasmonic sensor," Nano Lett. 10(7), 2342-2348 (2010).

7. A. Manjavacas, and F. J. García de Abajo, "Tunable plasmons in atomically thin gold nanodisks," Nat. Commun. 5, 3548 (2014).

8. A. Sihvola, Electromagnetic Mixing Formulas and Applications (The Institution of Electrical Engineers, 1999), Chap. 9.

9. H. T. Beyene, J. W. Weber, M. A. Verheijen, M. C. M. van de Sanden, and M. Creatore, "Real time in situ spectroscopic ellipsometry of the growth and plasmonic properties of Au nanoparticles on $\mathrm{SiO}_{2}$," Nano Res. 5(8), 513-520 (2012).

10. X. D. Li, T. P. Chen, Y. Liu, and K. C. Leong, "Influence of localized surface plasmon resonance and free electrons on the optical properties of ultrathin Au films: a study of the aggregation effect," Opt. Express 22(5), 5124-5132 (2014).

11. Y. Battie, A. E. Naciri, W. Chamorro, and D. Horwat, "Generalized effective medium theory to extract the optical properties of two-dimensional nonspherical metallic nanoparticle layers," J. Phys. Chem. C 118(9), 4899-4905 (2014).

12. T. Ung, L. M. Liz-Marzán, and P. Mulvaney, "Optical properties of thin films of $\mathrm{Au@SiO} \mathrm{S}_{2}$ particles," J. Phys. Chem. B. 105(17), 3441-3452 (2001).

13. J. Toudert, L. Simonot, S. Camelio, and D. Babonneau, "Advanced optical effective medium modeling for a single layer of polydisperse ellipsoidal nanoparticles embedded in a homogeneous dielectric medium: surface plasmon resonances,” Phys. Rev. B 86(4), 045415 (2012).

14. W.-J. Lee, J.-E. Kim, H.-Y. Park, S. Park, and M.-S. Kim, "Optical constants of evaporated gold films measured by surface plasmon resonance at telecommunication wavelengths," J. Appl. Phys. 103(7), 073713 (2008).

15. X. Wang, K.-P. Chen, M. Zhao, and D. D. Nolte, "Refractive index and dielectric constant evolution of ultra-thin gold from clusters to films," Opt. Express 18(24), 24859-24867 (2010).

16. J. Albert, L.-Y. Shao, and C. Caucheteur, “Tilted fiber Bragg grating sensors," Laser Photonics Rev. 7(1), 83-108 (2013).

17. M. Z. Alam, and J. Albert, "Selective excitation of radically and azimuthally polarized optical fiber cladding modes," J. Lightw. Technol. 31(19), 3167-3175 (2013).

18. W. Zhou, D. J. Mandia, M. B. E. Griffiths, A. Bialiayeu, Y. Zhang, P. G. Gordon, S. T. Barry, and J. Albert, "Polarization-dependent properties of the cladding modes of a single mode fiber covered with gold nanoparticles,” Opt. Express 21(1), 245-255 (2013). 
19. C. Caucheteur, C. Chen, V. Voisin, P. Berini, and J. Albert, "A thin metal sheath lifts the EH to HE degeneracy in the cladding mode refractometric sensitivity of optical fiber sensors," Appl. Phys. Lett. 99(4), 041118 (2011).

20. Y.-C. Lu, R. Geng, C. Wang, F. Zhang, C. Liu, T. Ning, and S. Jian, "Polarization effects in tilted fiber Bragg grating refractometers,” J. Lightw. Technol. 28(11), 1677-1684 (2010).

21. Y. Shevchenko, and J. Albert, "Plasmon resonances in Gold-coated titled fiber Bragg gratings," Opt. Lett. 32(3), 211-213 (2007).

22. Y. Shevchenko, C. Chen, M. A. Dakka, and J. Albert, "Polarization-selective grating excitation of plasmons in cylindrical optical fibers,” Opt. Lett. 35(5), 637-639 (2010).

23. J.-M. Renoirt, M. Debliquy, J. Albert, A. Ianoul, and C. Caucheteur, "Surface plasmon resonances in oriented silver nanwire coatings on optical fibers," J. Phys. Chem. C 118(20), 11035-11042 (2014).

24. W. Zhou, D. J. Mandia, M. B. E. Griffiths, S. T. Barry, and J. Albert, "Effective permittivity of ultrathin chemical vapor deposited gold films on optical fibers at infrared wavelengths," J. Phys. Chem. C 118(1), 670-678 (2014).

25. L.-Y. Shao, J. P. Coyle, S. T. Barry, and J. Albert, "Anomalous Permittivity and Plasmon Resonances of Copper Nanoparticle Conformal Coatings on Optical Fibers," Opt. Mater. Express 1(2), 128-137 (2011).

26. I. Horcas, R. Fernández, J. M. Gómez-Rodríguez, J. Colchero, J. Gómez-Herrero, and A. M. Baro, "WSXM: A software for scanning probe microscopy and a tool for nanotechnology," Rev. Sci. Instrum. 78(1), 013705 (2007).

27. S. Kedenburg, M. Vieweg, T. Gissibl, and H. Giessen, "Linear refractive index and absorption measurements of nonlinear optical liquids in the visible and near-infrared spectral region," Opt. Mater. Express 2(11), 1588-1611 (2012).

28. J. Lin, and C. W. Brown, "Near-IR spectroscopic determination of $\mathrm{NaCl}$ in aqueous solution," Appl. Spectrosc. 46(12), 1809-1815 (1992).

29. A. Yariv, and P. Yeh, Photonics: Optical Electronics in Modern Communications, 6th ed. (Oxford University Press, 2007), Chap. 3.2.

30. P. B. Johnson, and R. W. Christy, "Optical constants of the noble metals," Phys. Rev. B 6(12), 43704379 (1972).

31. A. Kossoy, V. Merk, D. Simakov, and K. Leosson, "Optical and structural properties of ultra-thin gold films," Adv. Opt. Mater. (to be published), doi: 10.1002/adom.201400345.

32. S. Laref, J. Gao, A. Asaduzzaman, K. Runge, P. Deymier, R. W. Ziolkowski, M. Mitawaki, and K. Muralidharan, "Size-dependent permittivity and intrinsic optical anisotropy of nanometric gold thin films: a density functional theory study,” Opt. Express 21(10), 11827-11838 (2013). 
33. S. D. Campbell, R. W. Ziolkowski, J. Cao, S. Laref, K. Muralidharan, and P. Deymier, "Anisotropic permittivity of ultra-thin crystalline Au films: Impacts on the plasmonic response of metasurfaces," Appl. Phys. Lett. 103(9), 091106 (2013).

34. J. C. Maxwell Garnett, "Colours in metal glasses and in metallic films," Phil. Trans. R. Soc. Lond. A 203, 385-420 (1904).

35. D. A. G. Bruggeman, "Berechnung verschiedener physikalischer Konstanten von heterogenen Substanzen. I. Dielektrizitätskonstanten und Leitfähigkeiten der Mischkörper aus isotropen Substanzen,” Ann. Phys. 416(7), 636-664 (1935).

36. S. B. Jones, and S. P. Friedman, "Particle shape effects on the effective permittivity of anisotropic or isotropic media consisting of aligned or randomly oriented ellipsoidal particles," Water Resour. Res. 36(10), 2821-2833 (2000).

37. R. W. Cohen, G. D. Cody, M. D. Coutts, and B. Abeles, "Optical properties of granular silver and gold films," Phys. Rev. B 8(8), 3689-3701 (1973).

38. U. Kreibig, and M. Vollmer, Optical Properties of Metal Clusters (Springer, 1995), Chap. 4.4.

39. M. Walther, D. G. Cooke, C. Sherstan, M. Hajar, M. R. Freeman, and F. A. Hegmann, "Terahertz conductivity of thin gold films at the metal-insulator percolation transition," Phys. Rev. B 76(12), 125408 (2007).

40. Y. Yagil, P. Gadenne, C. Julien, and G. Deutscher, "Optical properties of thin semicontinuous gold films over a wavelength range of 2.5 to $500 \mu \mathrm{m}$," Phys. Rev. B 46(4), 2503-2511 (1992). 


\section{Chapter 6: Optical Anisotropy of Ultrathin Gold}

\section{Evaporation Coatings on Optical Fibers (Further Work)}

In this chapter, we show preliminary results of investigations in the anisotropy of the optical properties of the ultrathin gold evaporated films (with mass-equivalent thicknesses from 3 to 8 $\mathrm{nm}$ ) during the insulator-to-metal transition process, based on the polarization-dependent spectral responses of the coated TFBGs. Results indicate that the optical properties of the $8 \mathrm{~nm}$ gold film are "metallic-like" in the in-plane direction, while the out-of-plane direction still shows insulating properties. Thus, growing gold films by PVD show a potentially lower percolation threshold for the in-plane direction than that for the out-of-plane direction.

\subsection{Introduction}

As one of the best deposition techniques for curved substrates, the gold chemical vapor deposition (CVD) can deposit uniform film on optical fiber surface. However, the gold CVD films reported in Chapter 4 do not support SPP propagation, due to the positive effective permittivity of the aggregated gold films. So the insulator-to-metal transition around the percolation threshold during the regular gold film growth (such as gold evaporation) does not appear in the gold CVD process, which indicates that the non-metallic properties of the gold CVD films remain up to thicknesses of at least $65 \mathrm{~nm}$. In order to investigate the permittivity evolution of ultrathin gold films including the insulator-to-metal transition process, we have to use a gold deposition method that can yield the continuous film morphology with conductive properties at small thicknesses. Based on the calculated anisotropic effective permittivity of 5.5 nm gold evaporation film reported in Chapter 5, the polarized TFBG cladding modes can be used for investigating the effective optical properties of the ultrathin gold films in both of in-plane and 
out-of-plane directions. And the $5.5 \mathrm{~nm}$ thick gold evaporation film that is close to the regular percolation threshold of the gold evaporation film around $6.5 \mathrm{~nm}$, showed some dipole interactions between the gold aggregates in in-plane direction, which indicate the delocalization effect of free electrons in the aggregated gold film with small-scale coalescence. Therefore, it was decided to investigate this further by using the gold evaporation deposition method for $e x$ situ investigation of the anisotropic insulator-to-metal transition (complex effective permittivity) during ultrathin gold film growth (thickness range from 3 to $8 \mathrm{~nm}$ ) with polarized TFBG cladding modes.

\subsection{Experiments and results (preliminary)}

In order to have a relatively uniform gold coating around the fiber circumference, two gold deposition steps were conducted consecutively with the fiber being rotated by exactly $180^{\circ}$ between the two deposition runs using a custom designed fiber holding fixture. However, due to the highly directional gold vapor during the deposition, it is impossible to get an absolutely uniform gold coating on optical fiber surface. So the orientation of the grating planes in TFBG was determined prior to mounting the TFBG in the deposition chamber by observing the diffraction pattern of visible light (red light) injected into the TFBG. Fig. 6.1 shows a typical diffraction pattern of the red light that propagates through the TFBG, where the two brightest spots in the vertical direction indicate the tilt orientation of the grating planes. Based on the simulated electric field distributions of the TE- and TM-polarized cladding modes shown in Fig. 5.1, the HE and EH modes have the strongest fields on the cladding boundary above and under the grating planes, and little or no intensity in the horizontal (sideways) directions. Thus, all TFBGs were fixed on the fiber holder in the gold deposition chamber with their grating planes 
oriented towards the rising gold vapor. As a result, the gold coatings on cladding surfaces facing the grating planes have the largest thickness.

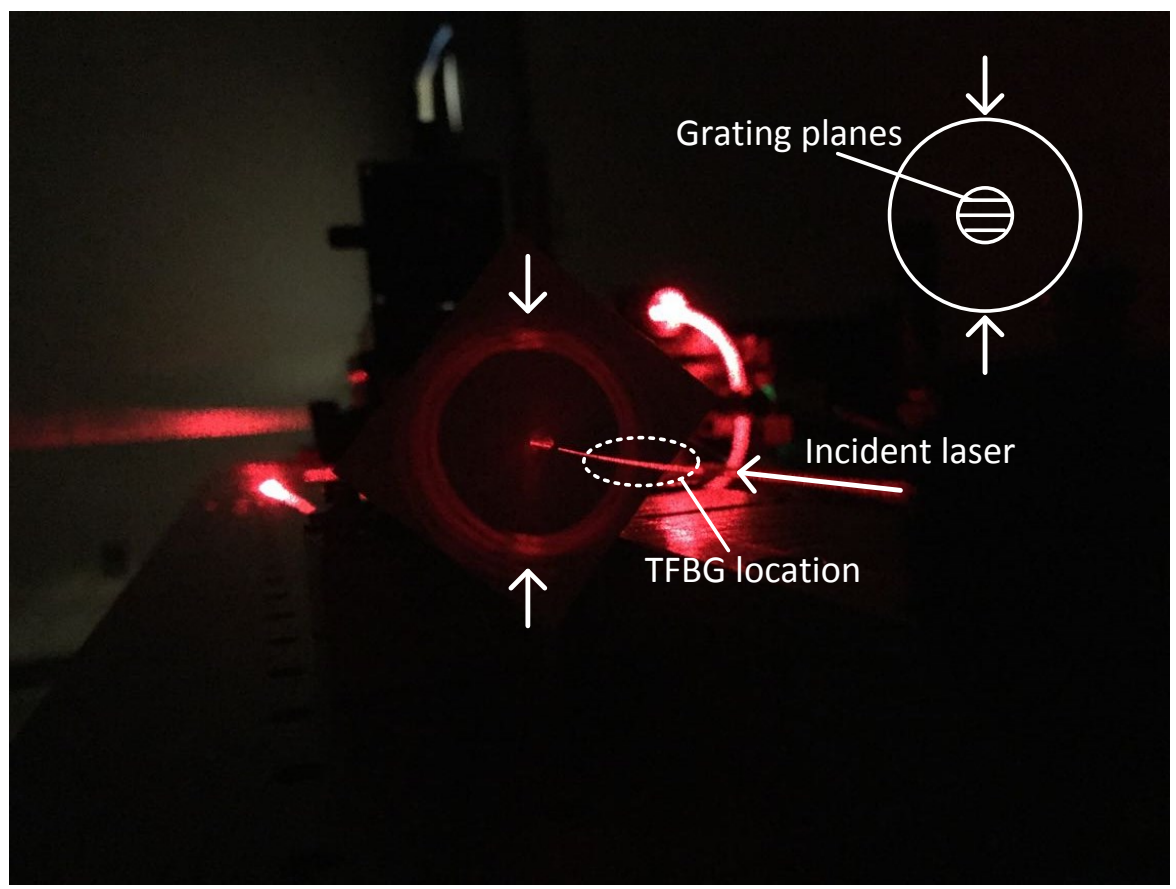

Fig. 6.1. Diffraction pattern of $10^{\circ} \mathrm{TFBG}$ induced by red incident light. The white arrows indicate the brightest areas along the diffraction pattern, where the TFBG planes are tilted away from the arrow direction.

In Fig. 6.2, we show the spectra of two TFBGs coated by the same $4 \mathrm{~nm}$ thick gold films, but with thickest coatings on the cladding surfaces vertical (a) and horizontal (b) to the grating planes. It is obvious that the cladding modes have much larger attenuations for case of thickest gold coatings on the vertical cladding surfaces, while have very weak responses for the case of thickest gold coating on the horizontal cladding surfaces. Therefore, in order to get the equivalent TFBG spectral responses caused by the uniform gold coating, all TFBGs in this work were coated by the gold films with the thickest areas vertical to the grating planes (such as the case shown in Fig. 6.2(a)). 

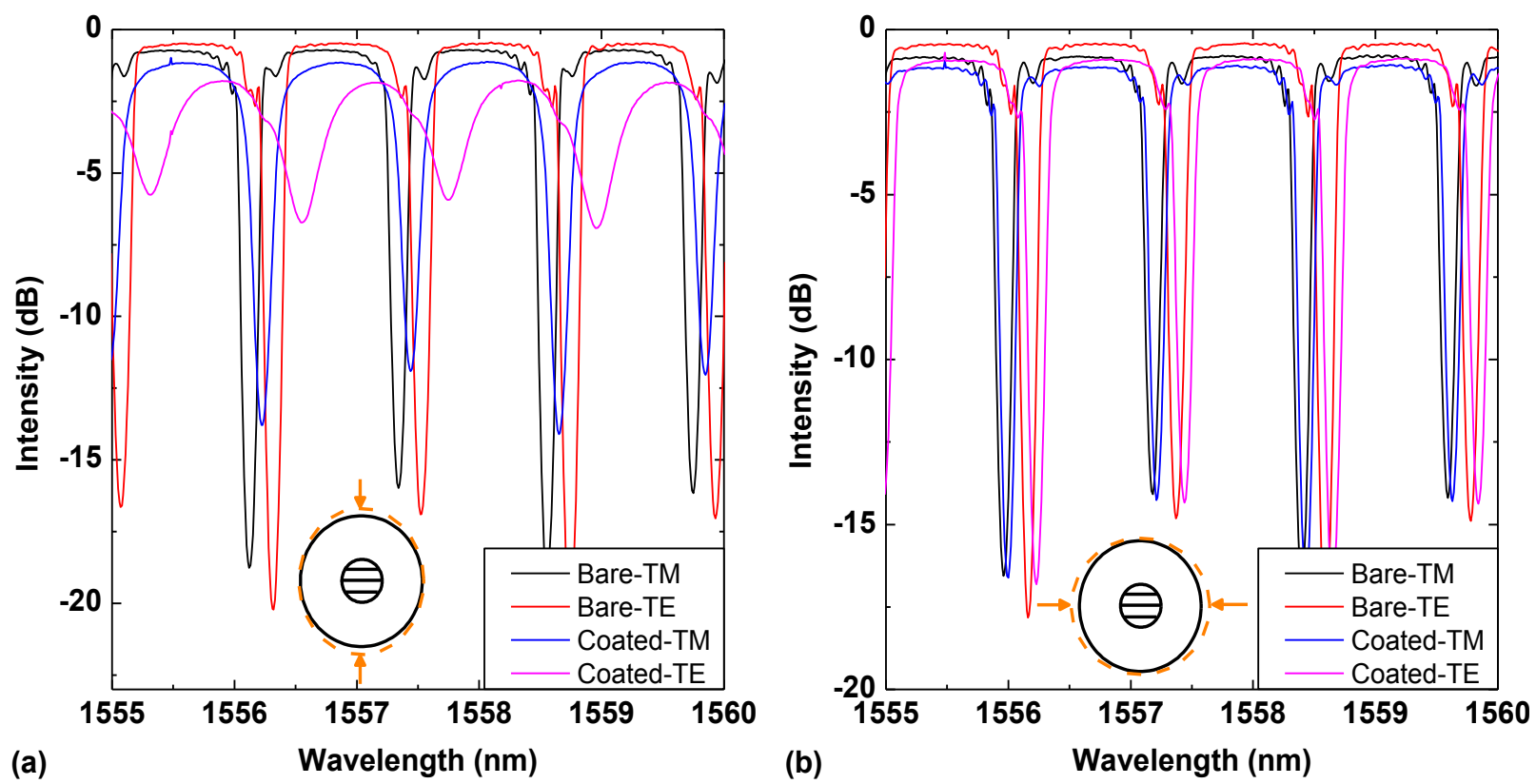

Fig. 6.2. Polarized spectra of cladding mode resonances in bare and $4 \mathrm{~nm}$ gold-coated $10^{\circ} \mathrm{TFBGs}$ with Bragg wavelength around $1610 \mathrm{~nm}$. The cross sections of two coated optical fibers show the two critical cases of the thickness profiles of the gold coatings relative to the TFBGs' orientations. The pair of arrows in each figure indicates the directions of the gold vapor in the double depositions.

For each gold deposition run on optical fiber samples, some witness silicon wafers with $30 \mathrm{~nm}$ silica buffer layers were placed next to the TFBG samples. These witness samples were used for SEM imaging of the coatings' morphology. Fig. 6.3 shows the SEM images of the gold evaporated films with mass-equivalent thicknesses from $3 \mathrm{~nm}$ to $8 \mathrm{~nm}$. It can be seen that both of the $3 \mathrm{~nm}$ and $4 \mathrm{~nm}$ gold films are completely composed of the irregular gold nanoislands, but with the increasing average size and irregularity. The large-scale coalescence of the aggregated gold nanoislands is obviously appearing in the $5 \mathrm{~nm}$ gold film, where some elongated gold structures are also formed by the coalescent gold aggregates. And the gold coalescences continue to connect with the adjacent ones in the $6 \mathrm{~nm}$ and $7 \mathrm{~nm}$ gold films, which result in a semicontinuous gold film. The inner percolating channels for the free electrons are gradually formed in the gold films during the coalescence process. The fraction of air voids is further 
reduced in the $8 \mathrm{~nm}$ gold film, of which the metallic properties are enhanced. Eventually, the air voids will fully be replaced with the gold material in thickness growth, leading to a continuous gold film with bulk properties.

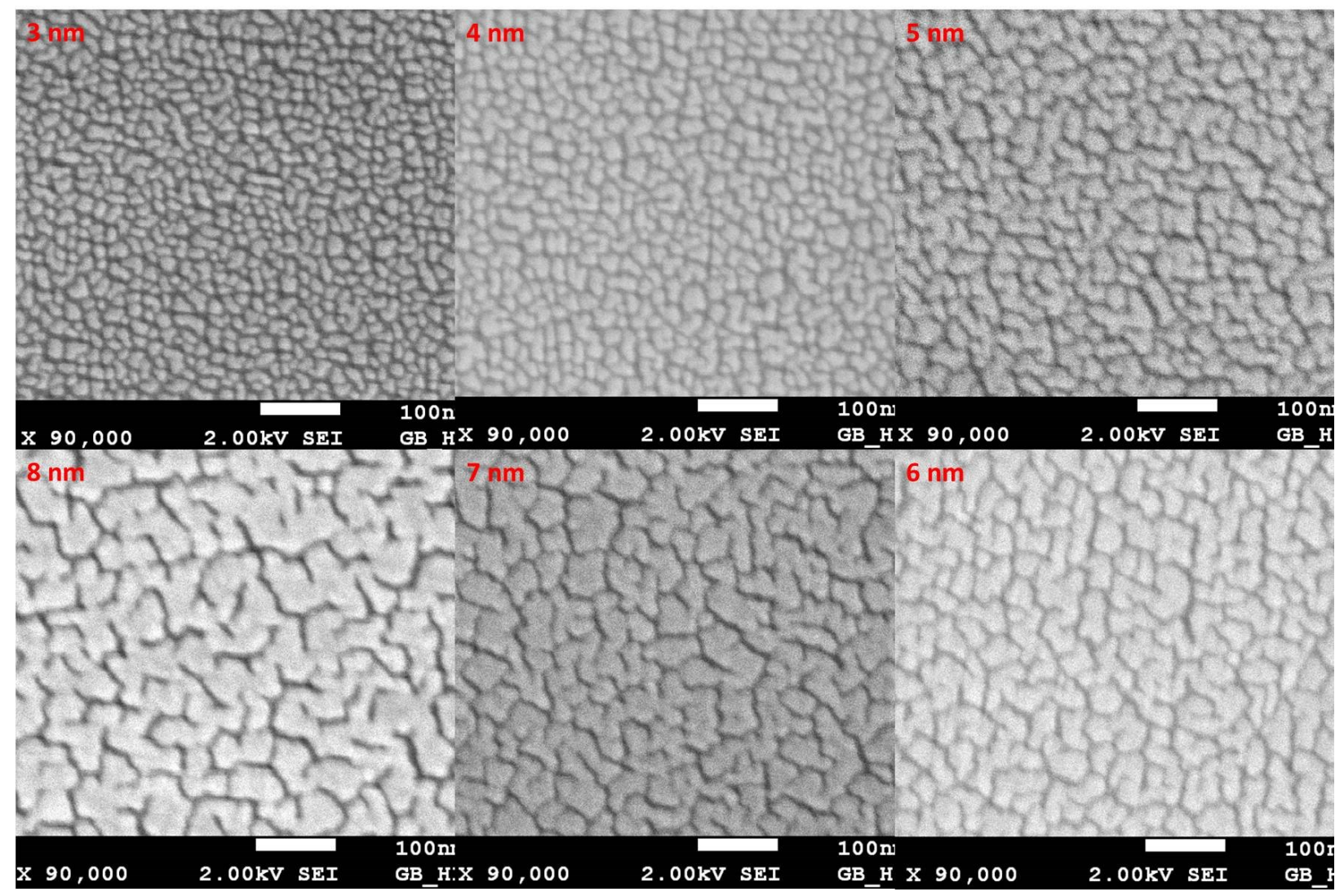

Fig. 6.3. SEM images of gold evaporation films deposited on witness wafers (all images were taken under the identical SEM parameters). Each image is marked with the mass-equivalent thickness. The scale bars indicate $100 \mathrm{~nm}$.

Fig. 6.4 shows the experimental wavelength shifts and amplitude attenuations of a pair of cladding modes with deepest spectral resonances around $1560 \mathrm{~nm}$, extracted from the bare TFBGs and the TFBGs coated by gold films with thickness from 3 to $8 \mathrm{~nm}$. In general, both of the polarized cladding modes show the increased attenuations with the gold coating growth, where the slight attenuation reduction of the TE mode for the thicknesses of 6 and $7 \mathrm{~nm}$ is mainly caused by the smaller initial amplitude of the resonance. It is important to note that 
identifying and following the cladding mode resonances under the particular polarization are difficult, especially for the gold films thicker than $4 \mathrm{~nm}$, due to the non-monotonic wavelength shifts and amplitude changes caused by the growing gold films. In order to confirm the extracted spectral data of the two polarized cladding modes shown in Fig. 6.4, more TFBG samples with gold coatings of other thicknesses between 3 and $8 \mathrm{~nm}$ are required in the future experiments.

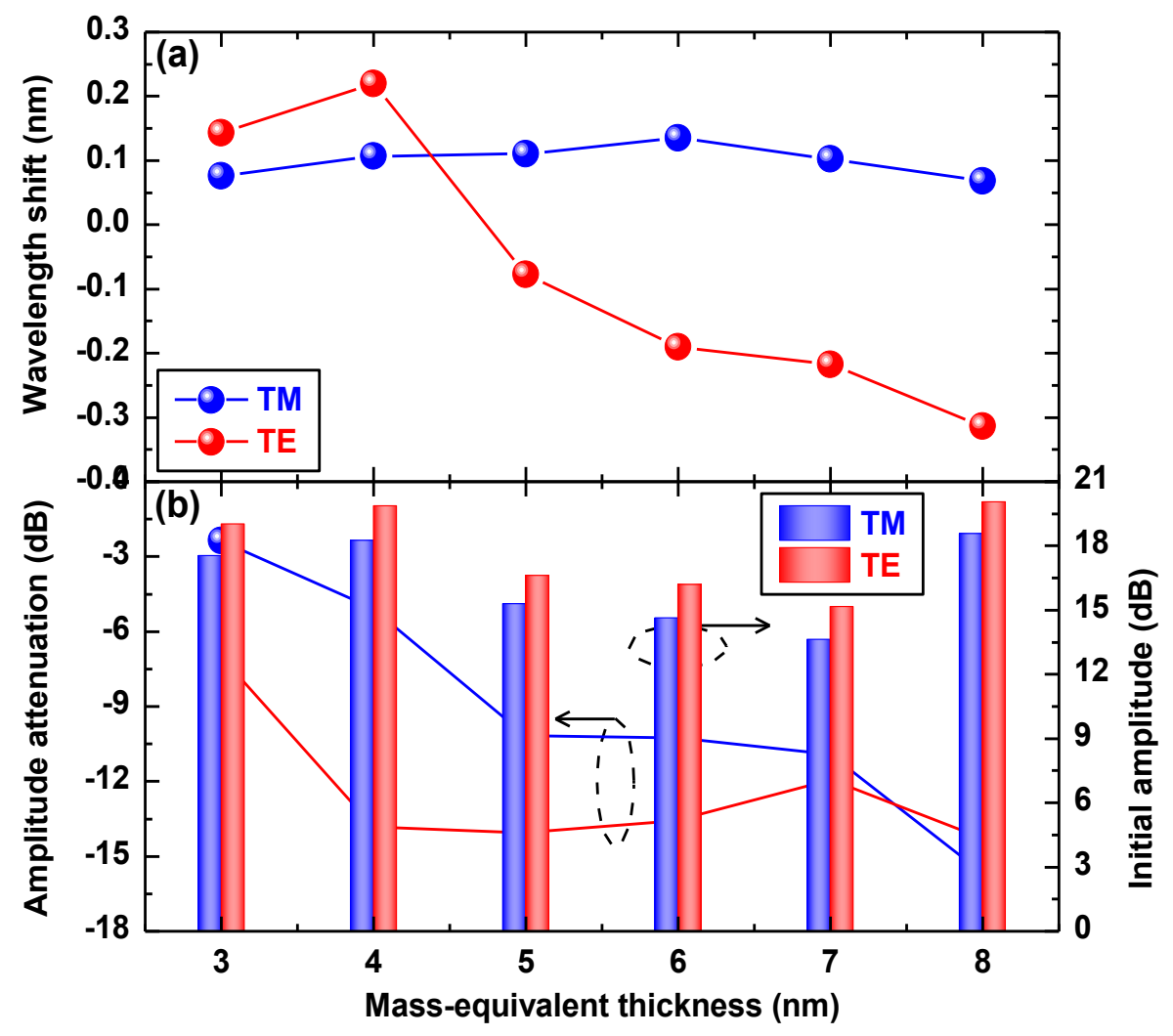

Fig. 6.4. Relative wavelength shifts (a) and amplitude attenuations (b) of TE- and TM-polarized cladding mode resonances versus the mass-equivalent thickness of gold film. The initial amplitudes of the pair of cladding modes in bare TFBGs are shown as a histogram in Fig. 6.4(b). 


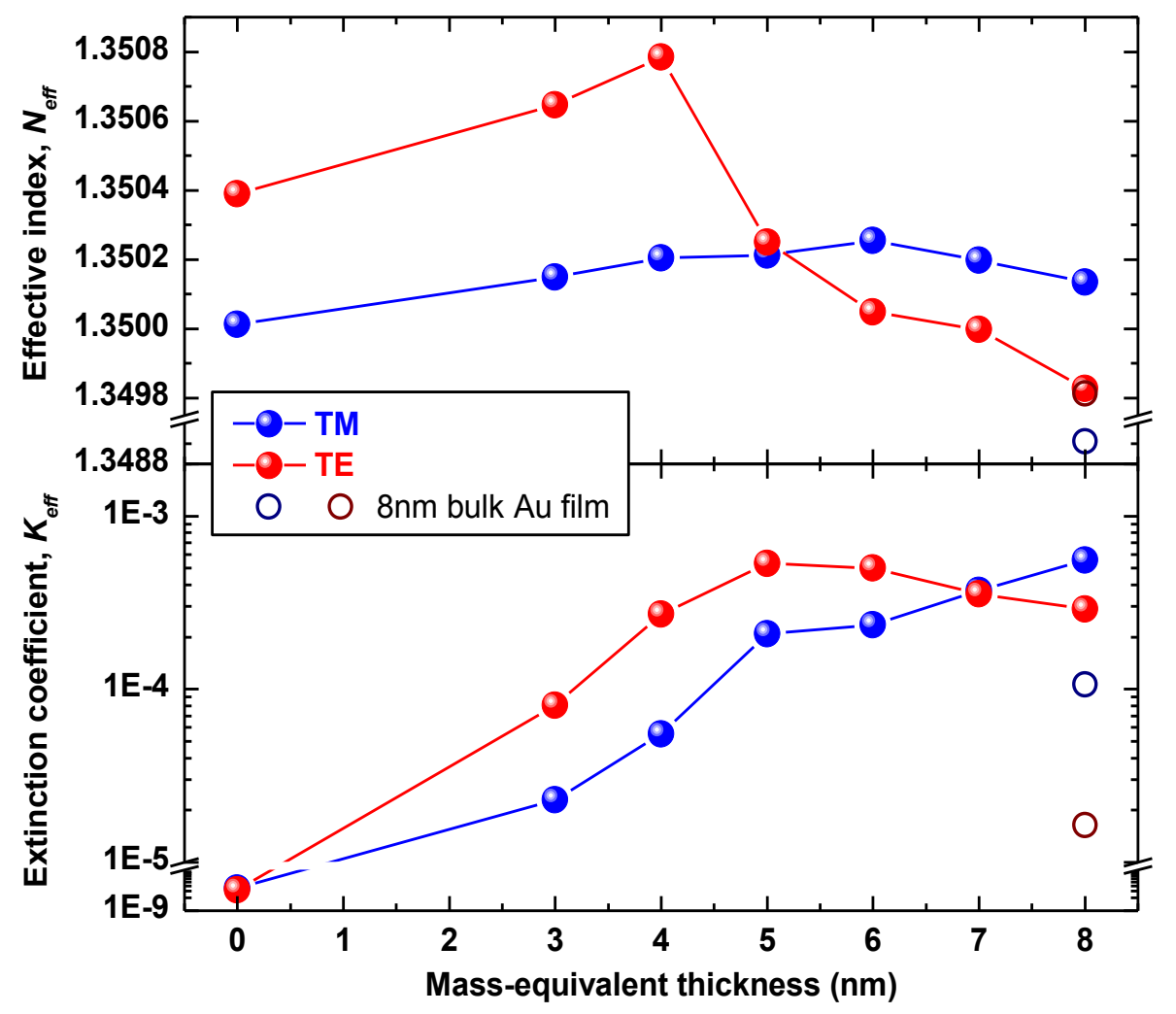

Fig. 6.5. Real (a) and imaginary (b) parts of the mode effective indices for the TE- and TM-polarized cladding modes measured in Fig. 6.4, versus the thickness of the gold film. The simulated complex refractive indices of the two polarized cladding modes for the $8 \mathrm{~nm}$ bulk gold coating are indicated by circles.

By using the data extraction algorithm described in Section 4.3 of Chapter 4 , the complex effective indices of the polarized cladding modes for the gold-coated TFBGs can be obtained. Fig. 6.5 shows the calculated real and imaginary parts of the mode indices under the different thickness of the gold coatings. In order to demonstrate the optical anisotropy of such ultrathin gold films, the effective indices of the corresponding cladding modes are simulated with a model of an optical fiber waveguide coated by $8 \mathrm{~nm}$ isotropic gold film with a bulk effective permittivity. From Fig. 6.5, the simulated real part for the TE-polarized mode is almost matched to the measured value, indicating the similar metallic properties of the $8 \mathrm{~nm}$ gold film in the in- 
plane direction. However, the measured real part of the mode index for the TM mode is much larger than the corresponding simulated value, showing that the optical properties of the $8 \mathrm{~nm}$ gold film are quite different from that of bulk gold for the out-of-plane direction. And it can be even supposed that the $8 \mathrm{~nm}$ gold film still performs as an insulator in the out-of-plane direction, based on the measured real part being bigger than that for bare TFBG (starting point of blue dots in Fig. 6.5(a)). On the other side, the simulated imaginary parts for $8 \mathrm{~nm}$ bulk gold coating fail to reproduce the measurement results, with underestimated magnitudes. This is due to the fact that the simulated imaginary parts of the effective indices are only dependent on the absorption coefficient of the uniform gold coating, while the measurements of mode loss, from which the experimental effective medium properties are derived, include a strong scattering component and is therefore larger than modelled. The NIR scattering effect can be quantitatively estimated by measuring the scattering light intensity from a part of gold coating and calculating the total intensity integrated over the full length and circumference of the TFBG.

\subsection{Summary}

In this chapter, we described preliminary results of the optical anisotropy of the percolating gold coatings with mass-equivalent thickness from $3 \mathrm{~nm}$ to $8 \mathrm{~nm}$, investigated with the polarized cladding modes of TFBG. The gold coatings were deposited on the optical fiber surfaces oriented along the tilted orientation of grating planes by thermal gold evaporation. The wavelength shifts and amplitude attenuations of the TE- and TM-polarized cladding modes caused by the gold coatings were extracted from the measured TFBG spectra. And the corresponding effective mode indices of the polarized cladding modes in the gold-coated TFBGs were calculated for demonstrating the optical anisotropy of the gold coatings around the classical percolation threshold around $6.5 \mathrm{~nm}$. In the future, we will focus on the two main issues about 
this work: 1. Orientation-dependent TFBG spectral responses to the non-uniform gold coatings with thickness profiles relative to the direction of titled grating planes. Are the spectral changes of the uniform gold film-coated TFBG close to the one coated by the double gold depositions that have thickest gold coatings on the cladding surfaces above and below the grating planes? 2 . How to distinguish the effects of absorption and scattering of the gold coatings on the attenuations of the polarized cladding modes. Eventually, it is hoped that the full the effective optical properties of the gold evaporated films around the percolation threshold can be investigated by using the TFBG-based anisotropic model. 


\section{Chapter 7: Conclusion}

A TFBG is a periodic structure inscribed in an optical fiber core by UV laser irradiation, which can couple the core mode into the SRI-dependent cladding modes. Due to the breakup of the circular symmetry caused by the tilted grating planes, the polarization state of the incident core mode controls the orientation and polarization of the excited cladding modes at the cladding boundary. In this PhD thesis, the TFBG probe is used as a bulk witness sensor for monitoring the thin gold film growth on fiber substrate and an "ellipsometry" tool for measuring the effective optical properties of the gold coatings on optical fiber surface.

Firstly, we have demonstrated that there is a significant correlation between the near infrared polarized transmission spectra of $10^{\circ}$ TFBG inscribed in standard single mode fibers and the optical properties gold particle coatings with thicknesses ranging from zero to $\sim 200 \mathrm{~nm}$, and formed using a recently developed CVD process. In particular we identified spectral signatures corresponding to coatings yielding different states of light confinement, scattering, and polarization. These effects are discussed in terms of the evolving metallic boundary conditions perceived by the cladding modes as the nanoparticles grow. This allows the preparation of gold nanoparticle layers that strongly discriminate between radially and azimuthally polarized cladding mode evanescent fields, with important consequences in the plasmonic properties of these layers. As a side benefit, we have also confirmed the usefulness of the TFBG as a process monitor for thin film coatings of CVD gold on other substrates in the same process chamber, in situ and in real time.

Secondly, the cladding modes of an optical fiber were used to investigate the complex refractive index of CVD gold coatings on the fiber cladding surface. The real and imaginary parts of the refractive index (and permittivity) of the gold nanoparticle films differ markedly 
from the bulk values at infrared wavelengths, as was often observed for films with thicknesses below $10 \mathrm{~nm}$. However in the case reported here, the anomalous values remain for thicknesses up to $65 \mathrm{~nm}$ and beyond, as in the case of evaporated gold films formed at very slow rates. It is expected that the real part of the complex refractive index reaches a saturated value at $65 \mathrm{~nm}$ then remains constant for higher thickneses, while the imaginary part would continue increasing due to the enhanced scattering and absorption effects during the thickness growth of gold CVD coating. The in-plane and out-of-plane components of the film permittivity were measured separately and shown to be essentially equal, apart from a small increasing dichroism at thicknesses larger than $25 \mathrm{~nm}$. The polarization-dependent refractive index of the metal-insulator mixture calculated by generalized Maxwell-Garnett EMA theory indicates that the gold CVD film consists of ellipsoidal particles with aspects ratios from 0.8 to 1.0 and air gaps corresponding to metal filling fractions increasing from $30 \%$ to $68 \%$ over the average thickness range from 6 to $65 \mathrm{~nm}$. Thus, it was demonstrated that the TFBG device can be used for the characterization at near infrared wavelengths of ultrathin metal films deposited by gas-phase processes, down to thicknesses below $10 \mathrm{~nm}$. The small TFBG probe can be inserted easily into deposition chambers to ensure that the film deposited on the probe is identical to that on adjacent substrates.

Thirdly, the SRI-dependent effective refractive indices of the anisotropic $5.5 \mathrm{~nm}$ gold NPs film deposited by gold evaporation method were investigated with the polarized cladding modes excited by a TFBG. Such an $5.5 \mathrm{~nm}$ anisotropic gold film enhances the surrounding refractive index sensitivity from $1.76 \mathrm{~nm} / \mathrm{RIU}$ to $2.49 \mathrm{~nm} / \mathrm{RIU}$ for the TE-polarized cladding mode, while slightly decreasing the sensitivity from $2.20 \mathrm{~nm} / \mathrm{RIU}$ to $2.14 \mathrm{~nm} / \mathrm{RIU}$ for the TMpolarized mode. The complex refractive indices for modes polarized in the in-plane (TE) and 
out-of-plane (TM) directions, and the corresponding effective thin coating parameters were extracted from the measured polarization-dependent wavelength shifts and amplitude changes of the cladding modes around $1570 \mathrm{~nm}$ with help from simulations carried out with an accurate four-layer complex mode solver. The slightly weaker insulating optical properties of the gold NPs film for the TE-polarization than those for TM-polarization suggest the proximity of an insulator-to-metal transition and a critical thickness lower than $5.5 \mathrm{~nm}$ at $1570 \mathrm{~nm}$, for the deposition conditions used. Moreover, comparing the experimental results with predictions from the generalized M-G and Bruggeman EMAs, it was revealed that these effective medium models fail to correctly account for the properties of the NP films at such thickness for near infrared wavelengths. It is interesting to note that for an ultrathin (less than $10 \mathrm{~nm}$ thick) silver films with significant non-uniformity, i.e. "islandized" into nanoclusters, it was shown experimentally that long range surface waves could be excited at visible wavelengths [1]. This indicates that further investigations of our ultrathin gold films (CVD and evaporated) would be useful provided that TFBGs operating at visible wavelengths could be fabricated.

Fourthly, we show the preliminary results about the investigations of the anisotropy of the optical properties of the ultrathin gold evaporation films (with mass-equivalent thickness from 3 to $8 \mathrm{~nm}$ ) during the insulator-to-metal transition process, based on the polarizationdependent spectral responses of the coated TFBGs. It indicates that the optical properties of the 8 $\mathrm{nm}$ gold film perform metallic in the in-plane direction, while the out-of-plane direction still shows insulating properties. Thus, for the growing gold evaporated films, it shows a potential lower percolation threshold for the in-plane direction than that for the out-of-plane direction.

Finally, due to the anomalous optical properties (poor conductivity) of the gold CVD films and ultrathin gold evaporation films, a TFBG-based SPR sensor for surrounding refractive 
index detection could not be obtained. However, the LSPR can be potentially excited in the gold nanoparticles and nanoislands on TFBG surface at visible wavelengths, which gives us some ideas for TFBG-based LSPR sensors. Furthemore, while the work carried out here dealt exclusively with complex permittivity materials (metals), the TFBG thin film probe that was developed can be used for isotropic and anisotropic dielectric coatings, a "simpler" problem in the sense that there are fewer unknowns. In that sense, it is hoped that at some point the TFBG probing technique will be able to provide the same kind of information as an ellipsometer, with the advantage that it can be inserted into deposition chambers for in situ, real time monitoring of thin films.

\section{References}

1. Fuzi Yang, G. W. Bradberry, and J. R. Sambles, "Long-range surface mode supported by very thin silver films," Physical Review Letters 66(15), 2030-2032 (1991). 


\section{Author's Research Output during PhD (2012.02-2015.02)}

\section{Publications in peer-reviewed journals:}

1. W. Zhou, D. J. Mandia, S. T. Barry, and J. Albert, “Anisotropic effective permittivity of an ultrathin gold coating on optical fiber in air, water, and saline solutions," Opt. Express 22(26), 31665-31676 (Dec. 2014).

2. †D. J. Mandia, W. Zhou, J. Albert, and S. T. Barry, "Chemical vapor deposition on optical fibers: tilted fiber Bragg gratings as real-time sensing platforms," Chem. Vap. Deposition (Early View) (Oct. 2014). doi: 10.1002/cvde.201400059

3. C. Shen, W. Zhou, and J. Albert, "Polarization-resolved evanescent wave scattering from gold-coated tilted fiber gratings,” Opt. Express 22(5), 5277-5282 (Mar. 2014).

4. C. Shen, Y. Zhang, W. Zhou, and J. Albert, "Au-coated tilted fiber Bragg grating twist sensor based on surface plasmon resonance," Appl. Phys. Lett. 104(7), 071106 (Feb. 2014).

5. ${ }^{\dagger}$ W. Zhou, D. J. Mandia, M. B.E. Griffiths, S. T. Barry, and J. Albert, "Effective permittivity of ultrathin chemical vapor deposited gold films on optical fibers at infrared wavelengths," $J$. Phys. Chem. C 118(1), 670-678 (Jan. 2014).

6. ${ }^{\dagger} D$. J. Mandia, M. B.E. Griffiths, W. Zhou, P. G. Gordon, J. Albert, and S. T. Barry, “In situ deposition monitoring by a tilted fiber Bragg grating optical probe: probing nucleation in chemical vapour deposition of gold," Phys. Procedia 46, 12-20 (2013).

7. W. Zhou, D. J. Mandia, M. B.E. Griffiths, A. Bialiayeu, Y. Zhang, P. G. Gordon, S. T. Barry, and J. Albert, "Polarization-dependent properties of the cladding modes of a single mode fiber covered with gold nanoparticles," Opt. Express 21(1), 245-255 (Jan. 2013).

8. X. Dong, Y. Zhou, W. Zhou, J. Cheng, and Z. Su, "Compact anemometer using silver-coated fiber Bragg grating,” IEEE Photon. J. 4(5), 1381-1386 (Oct. 2012).

9. W. Zhou, Y. Zhou, X. Dong, L.-Y. Shao, J. Cheng, and J. Albert, "Fiber-optic curvature sensor based on cladding-mode Bragg grating excited by fiber multimode interferometer," IEEE Photon. J. 4(3), 1051-1057 (Jun. 2012).

\section{Conference papers:}

1. 'D. J. Mandia, W. Zhou, M. J. Ward, H. Joress, J. B. Giorgi, P. Gordon, J. Albert, and S. T. Barry, "Chemical vapor deposition of anisotropic ultrathin gold films on optical fibers: real- 
time sensing by tilted fiber Bragg gratings and use of a dielectric pre-coating," Photonics North 2014, Proc. SPIE 9288, 92880M (2014).

2. ${ }^{\dagger}$ W. Zhou, D. J. Mandia, M. B.E. Griffiths, S. T. Barry, and J. Albert, “Anomalous refractive index of ultrathin gold nanoparticle film coated on tilted fiber Bragg grating," OFS2014 23rd International Conference on Optical Fibre Sensors, Proc. SPIE 9157, 91573Y (2014). $\dagger$ indicates that the publication is part of the PhD project. 